\author{
University of São Paulo \\ "Luiz de Queiroz" College of Agriculture
}

Tomato gynoecium patterning and fruit development are orchestrated by the interplay between microRNAs and gibberellin

\author{
João Paulo de Oliveira Corrêa
}

Thesis presented to obtain the degree of Doctor in

Science. Area: Plant Physiology and Biochemistry

Piracicaba 
João Paulo de Oliveira Corrêa

Bachelor in Biological Sciences

Tomato gynoecium patterning and fruit development are orchestrated by the interplay between microRNAs and gibberellin

Advisor:

Prof. Dr. FABIO TEBALDI SILVEIRA NOGUEIRA

Thesis presented to obtain the degree of Doctor in Science. Area: Plant Physiology and Biochemistry

Piracicaba

2019 


\section{Dados Internacionais de Catalogação na Publicação} DIVISÃO DE BIBLIOTECA - DIBD/ESALQ/USP

\section{Corrêa, João Paulo de Oliveira}

Tomato gynoecium patterning and fruit development are orchestrated by the interplay between microRNAs and gibberellinl / João Paulo de Oliveira Corrêa. - - Piracicaba, 2019.

$80 \mathrm{p}$.

Tese (Doutorado) - - USP / Escola Superior de Agricultura "Luiz de Queiroz".

1. Tomateiro 2. MiR156 3. Giberelina 4. Fruto 5. Gineceu I. Título 


\section{ACKNOWLEDGEMENTS}

I would like to acknowledge everyone that, directly and indirectly, contributed to my thesis. I am deeply thankful to you all. Special thanks to:

My supervisor Dr Fabio Nogueira for being a very good supervisor, always supportive, involved in my work and available for fruitful scientific discussions. Thank you very much for the patience and knowledge shared.

My supervisor in John Innes Centre, Dr Robert Sablowski. Thank you for hosting me in your lab, supporting my research and sharing all the amazing knowledge on meristem development. I learned so many things in this visit that those six months looked like a much longer period to me.

Dr Lázaro Peres for the scientific knowledge shared, seed greenhouse structure and lab equipment provided during my $\mathrm{PhD}$.

All my lab colleagues and friends from GMDV Antoine, Leticia, Diego, Carol, Mateus, Carlos and Felipe.

All colleagues and friends from LCHDV.

Geraldo Silva and Eder Silva for being practically my co-supervisors during my first $\mathrm{PhD}$ years and for the friendship we developed along the time. Thank you for the friendship, for teaching me from scratch how to work with molecular biology, for all the scientific discussion we always had.

Airton Carvalho and Marcela Notini for the friendship, help and for also being the guys who enjoy having a beer to talk about science.

My friends from John Innes Centre Marco, Emma, Max, Aura, Anna, Marina and Jesus. Thank you very much for the support, the knowledge shared and for making my adaptation to UK much easier. I am really thankful for meeting you all.

My friends Lorena, Andressa, Lucas and Italo for being my family in Piracicaba. For all the unforgettable moments we had together, in good times and bad times.

Danilo, for being always by my side giving me emotional support during the whole $\mathrm{PhD}$ time. Thank you very much for everything!

My parents Batista and Magda for absolutely everything. For all the love, for being examples of human beings to me.

My sister Marina for all the love and patience.

CNPq (process 140513/2015-9) and FAPESP (processes 2016/05073-4 and 2018/13316-0) for the financial support. 


\section{CONTENTS}





1. MOLECULAR CONTROL BY NON-CODING RNAS DURING FRUIT DEVELOPMENT: FROM GYNOECIUM PATTERNING TO FRUIT RIPENING ...............................................................................7

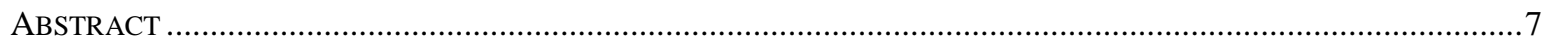

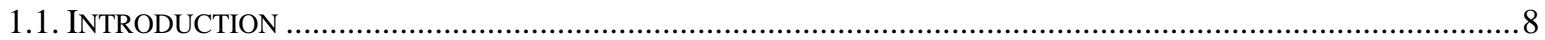

1.2. MICRORNA MODULES INVOLVED IN EARLY STEPS OF FRUIT PATTERNING AND GROWTH .............................

1.2.1. The miR164 module controls carpel development and leaf margin serration through similar

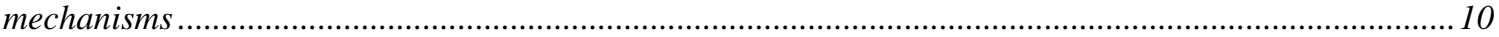

1.2.2. The role of miR156/miR157 in carpel and fruit development ..................................................... 12

1.2.3. miR396 module regulate CMM meristematic competence and pluripotency during gynoecium

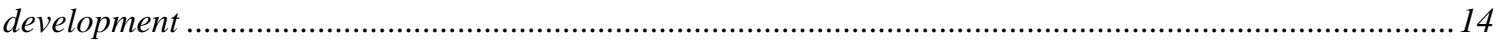

1.2.4. microRNA160 module controls carpel development by modulating auxin responses.......................16

1.2.5. miR172 limits the growth-repressing activity of APETALA2-like genes during fruit expansion ....... 17

1.3. MICRORNA-CONTROLLED PATHWAYS MODULATING OVULE AND SEED DEVELOPMENT DURING FRUIT

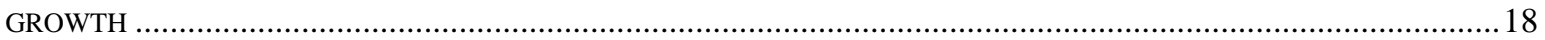

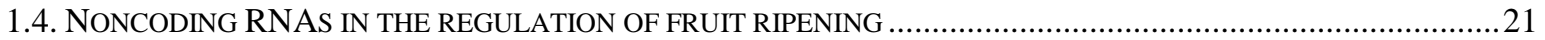

1.4.1. Conserved and Solanaceae-specific miRNA modules control tomato fruit ripening..........................21

1.4.2. New evidences of the role of long non-coding RNAs (lncRNAs) in fruit ripening.............................23

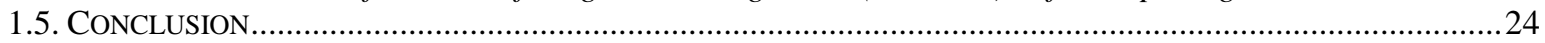

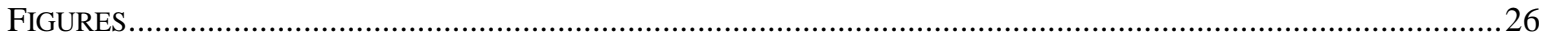

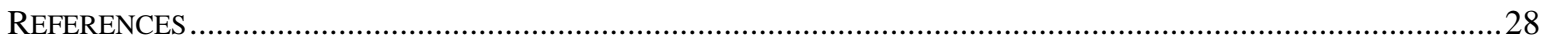

2. MIR156-TARGETED SPL/SBPS MODULATE GIBBERELLIN (GA) RESPONSES AND REGULATE GYNOECIUM DEVELOPMENT BY CONTROLLING BOUNDARY ESTABLISHMENT

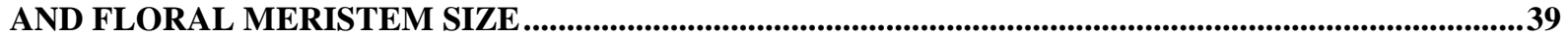

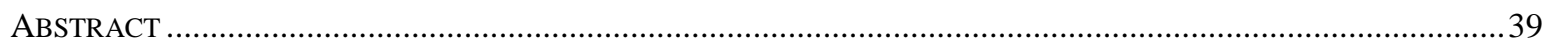

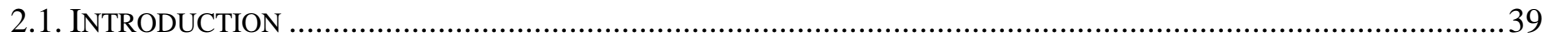

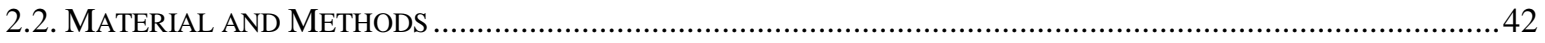

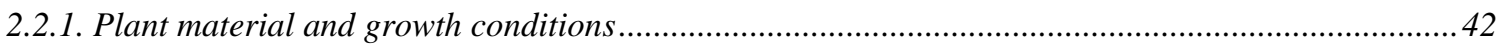



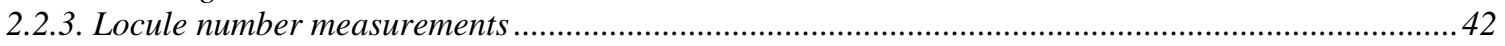

2.2.4. Generation of expression vectors and plant transformation .........................................................43

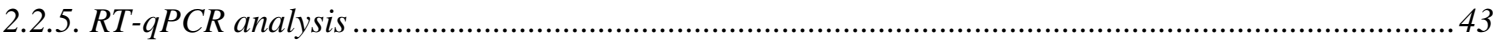

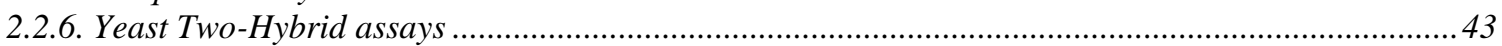

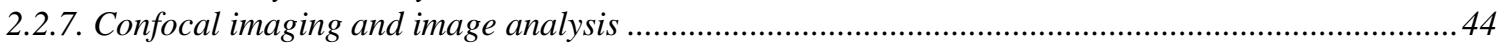

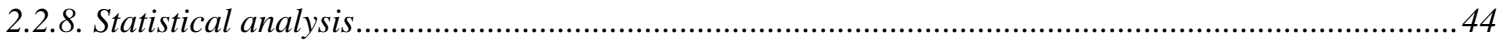



2.3.1. miR156 module and GA regulate fruit locule number and control GOBLET expression ..................44

2.3.2. Distinct miR156-targeted SBP/SPL transcription factors regulate locule number and GOBLET

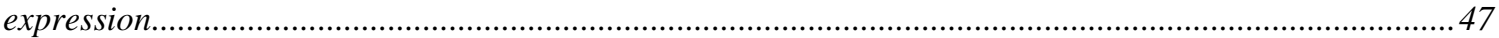

2.3.3. miR156 and GA synergistically regulate gynoecium development ................................................49

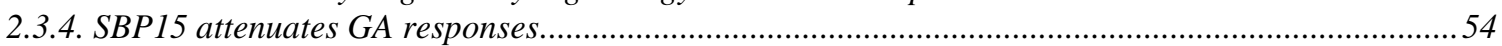

2.3.5. Among the miR156-targeted SPL/SBPs, SBP15 has a major role in controlling gynoecium

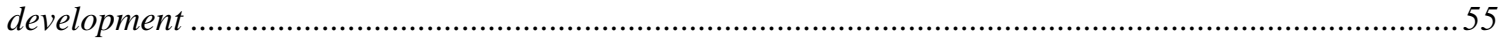

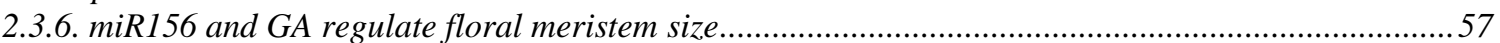





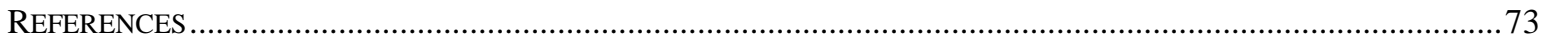


RESUMO

O desenvolvimento do gineceu e fruto em tomateiro é coordenado pela interação entre giberelina e microRNAs

\begin{abstract}
Muitas características finais deu um fruto são controladas por processos que se dão muito cedo no desenvolvimento dos frutos, até mesmo a nível de meristema. De fato, processos que vão desde a transição de um meristema vegetativo para um meristema de inflorescência até os estágios finais do amadurecimento definem muitas características do fruto maduro. Fatores de transcrição regulados pós transcricionalmente por microRNAs (miRNAs) têm papel crucial na maior parte desses estágios. Nessas vias os miRNAs geralmente regulam a expressão de seus alvos espacialmente, temporalmente, ou diminuem o acúmulo de transcritos do alvo, proporcionando níveis adequados de expressão para o desenvolvimento do órgão. Muitos estudos mostram que determinados miRNAs são essenciais no controle do tempo de florescimento, desenvolvimento do gineceu, crescimento do fruto após a polinização e amadurecimento, dentre outros estágios do desenvolvimento do fruto. Muitos aspectos do controle do desenvolvimento inicial do gineceu foram relativamente bem estudados em Arabidopsis. Porém, essas etapas iniciais do desenvolvimento de carpelos/gineceu ainda não são bem estudadas em plantas que produzem fruto carnoso, como é o caso do tomateiro. Já foi demonstrado que muitas vias reguladas por miRNAs interagem com fitormônios na regulação de vários processos de desenvolvimento. Como exemplo podemos citar a relação entre a via do miR156 e a via das giberelinas (GA) no controle do tempo de florescimento. Essa relação é substancialmente em Arabidopsis e tomateiro. Além disso, foi demonstrado que o miR156 possui papéis distintos na regulação do desenvolvimento do gineceu nessas duas espécies. Dessa forma, nesse trabalho nós: (1) fazemos uma revisão de literatura sobre a regulação do desenvolvimento de frutos, do desenvolvimento inicial do gineceu ao amadurecimento, por vias reguladas por RNAs não codantes e; (2) estudamos a relação entre as vias do miR156 e GA na regulação de etapas iniciais do desenvolvimento de frutos. Nós demonstramos que essas vivas controlam o tamanho de meristemas florais, bem como o estabelecimento de zonas de divisão no desenvolvimento do gineceu. Além disso, mostramos que a via do miR156 modula a resposta a GA.
\end{abstract}

Palavras-chave: Tomateiro; MiR156; Giberelina; Fruto; Gineceu 
ABSTRACT

Tomato gynoecium patterning and fruit development are orchestrated by the interplay between microRNAs and gibberellin

\begin{abstract}
Many characteristics of a fully developed fruit are controlled by processes that take place early in fruit development, even at floral meristem level. In fact, events that range from the transition of a vegetative into an inflorescence meristem to the last stages of fruit ripening determine characteristics of a mature fruit. Transcription factors post-transcriptionally regulated by microRNAs (miRNAs) play crucial roles in most of these stages. In such pathways, miRNAs may regulate its targets spatially, temporally, or dampen amount of targets transcript to provide optimal expression patterns for adequate organ development. Many miRNAs have been described to be essential in the regulation of flowering time, gynoecium patterning, post-pollination gynoecium growth and fruit ripening, among other stages of fruit development. Some aspects of early stages of gynoecium development, such as floral meristem maintenance, carpel fusion and gynoecium pattern, have been well studied in Arabidopsis. However, these stages are poorly understood in the development of fleshy fruit species, such as tomato. Many miRNA-related pathways were described to interact with phytohormone pathways. A good example is the interaction between miR156 and Gibberellins (GA) in the regulation of flowering time. Interestingly, this interaction is substantially different in Arabidopsis and tomato. MiR156 have been also shown to have distinct roles in controlling tomato and Arabidopsis gynoecium development. Considering this, here we: (1) review the available literature concerning control of fruit development, from gynoecium patterning to fruit ripening, by non-coding RNAs and (2) study the relationship between miR156 and GA pathways in the regulation of early stages of fruit development. We show that these pathways control floral meristem size and boundary establishment during gynoecium development and miR156 pathway modulates responses to GA.
\end{abstract}

Keywords: Tomato; MiR156; Gibberellin; Fruit; Gynoecia 


\title{
1. MOLECULAR CONTROL BY NON-CODING RNAS DURING FRUIT DEVELOPMENT: FROM GYNOECIUM PATTERNING TO FRUIT RIPENING
}

\author{
João Paulo de Oliveira Correa ${ }^{1,2}$, Eder M. Silva ${ }^{1,2^{*}}$, Fabio T. S. Nogueira ${ }^{1, \dagger}$ \\ ${ }^{1}$ Laboratory of Molecular Genetics of Plant Development, Department of Biological Sciences \\ (LCB), Escola Superior de Agricultura "Luiz de Queiroz" (ESALQ), University of Sao Paulo, \\ 13418-900 Piracicaba, Sao Paulo, Brazil.
}

${ }^{2}$ These authors contributed equally to this work.

${ }^{*}$ Current address: Laboratory of Plant Genetic Improvement, Centro de Energia Nuclear na Agricultura, Universidade de São Paulo, Piracicaba, SP 13400-970, Brazil.

${ }^{\dagger}$ To whom correspondence should be addressed:

Fabio T.S. Nogueira

ESALQ/USP

Avenida Padua Dias, 11

13418-900 Piracicaba, Sao Paulo, Brazil

Phone: (+55-19) 34294052 r.301

E-mail: ftsnogue@usp.br

Running title: Non-coding RNAs in fruit development and ripening

Invited review article published in Frontiers in Plant Science

\begin{abstract}
Fruits are originated from the transition of a quiescent ovary to a fast-growing young fruit. The evolution of reproductive structures such as ovary and fruit has made seed dispersal easier, which is a key process for reproductive success in flowering plants. The complete fruit development and ripening are characterized by a remarkable phenotypic plasticity which is orchestrated by a myriad of genetic factors. In this context, transcriptional regulation by noncoding small (i.e., microRNAs) and long (lncRNAs) RNAs underlie important mechanisms controlling reproductive organ development. These mechanisms may act together and interact with other pathways (i.e. phytohormones) to regulate cell fate and coordinate reproductive organ development. Functional genomics has shown that non-coding RNAs regulate a diversity of developmental reproductive stages, from carpel formation and ovary development to the softening of the ripped fruit. This layer of transcriptional control has been associated with ovule, seed, and fruit development as well as fruit ripening, which are crucial developmental processes in breeding programs because of their relevance for crop production. The final ripe fruit is the
\end{abstract}


result of a process under multiple levels of regulation, including mechanisms orchestrated by microRNAs and lncRNAs. Most of the studies we discussed involve work on tomato and Arabidopsis. In this review, we summarized non-coding RNA-controlled mechanisms described in the current literature that act coordinating the main steps of gynoecium development/patterning and fruit ripening.

Keywords: Tomato; Fruit development; MicroRNAs; IncRNAs; Ripening

\subsection{Introduction}

Fruits are plant organs found solely in angiosperms and are commonly defined as mature ovaries containing seeds. They are also ecologically defined as seed dispersal units, and their diversification and specialization are key events of the adaptive success of angiosperms in a wide range of environments (Seymour et al., 2013). The final characteristics of a mature fruit are determined by events that take place in developmental stages ranging from floral meristem initiation to later stages of fruit ripening. Complex mechanisms of transcriptional regulation of each of these stages ensure proper fruit development. After floral meristem initiation, key events of fruit development include carpel formation, differentiation, patterning and organ boundary formation. Ovule and seed development are also fundamental processes for the completion of fruit maturation. Fruit set occurs when the signaling triggered by the pollination and fertilization turns a fully developed ovary into a fast-growing fruit that will soon initiate the ripening process.

Although physiological and molecular aspects of fruit development and ripening are well discussed in the available literature (Ferrándiz et al., 2010; Liu et al., 2015), few reviews focused on the role of non-coding RNA-based molecular regulation controlling early and late stages of fruit development. Here, we reviewed the literature focused on the aspects of the regulation by non-coding RNA in different stages of fruit development, including ovule and seed development. Moreover, we discussed aspects of fruit growth and ripening in the light of miRNA and lncRNA-associated mechanisms. One important question that need to be better addressed in future studies is how transcriptional control of fruit development is conserved between dry fruitbearing and fleshy fruit-bearing species (e.g, Arabidopsis thaliana and tomato or Solanum lycopersicum, respectively). A better understanding of the non-coding RNA-related transcription hallmarks orchestrating early steps of fruit development and ripening in different species may have the potential to provide novel strategies for crop improvement. 


\section{2. microRNA modules involved in early steps of fruit patterning and growth}

The carpel is the female reproductive organ that encloses the ovules in flowering plants. The gynoecium is the innermost floral whorl, formed by the fusion of carpels in the center of the flower. The hypothesis of the origin of the carpels as modified leaves is corroborated by the observation that leaf development-associated factors also have roles in carpel development (Dinneny et al., 2005; Scutt et al., 2006; Alonso-Cantabrana et al., 2007; Ferrándiz et al., 2010; Gonzales-Reig et al., 2012; Seymour et al., 2013; Deb et al., 2018). Carpel and fruit development can be broadly divided into two main temporal set of events: an earlier set of events that occur prior to fertilization (differentiation and patterning), and later events, which occur after fertilization (growth, ripening and senescence) (Ripoll et al., 2015; Deb et al., 2018). A fine-tuned molecular regulation of each of these developmental steps is crucial to ensure proper morphological and physiological characteristics of the mature fruit.

MicroRNAs (miRNAs) and their targets (mostly transcription factors; Chen, 2009) are fundamental components of molecular modules (hereafter referred to microRNA modules) belonging to complex circuits that control various aspects of plant development. miRNAs inhibit the activity of their targets by two major mechanisms: ARGONAUTE1 (AGO1)-mediated transcriptional cleavage, and translational repression of gene targets (Borges and Martienssen, 2015). At cell and tissue levels, many miRNAs accumulate in a spatiotemporal manner to modulate and/or fine-tune the expression of their targets (Chen, 2009; Rubio-Somoza and Weigel, 2011). For instance, some miRNAs participate in tissue patterning by restricting the expression domain of target genes (Berger et al., 2009; Chen, 2009; Ripoll et al., 2015). On the other hand, miRNAs and targets may be co-expressed in similar domains, where miRNAs ensure proper transcript accumulation by dampening target transcript levels. In this case, miRNAs generally mediate the temporal control of transcript accumulation, in which cells and/or tissues exhibit a gradual decrease or increase in the levels of target transcripts as the organ develops (Wu et al., 2010; Rubio-Somoza et al., 2014; Wang, 2014; Guo et al., 2017; He et al., 2018).

Some miRNA modules had their roles in gynoecium and fruit development described in different model plants, such as Arabidopsis thaliana, which produces dry fruits (silique), and tomato (Solanum bycopersicum), which produces fleshy fruit (berry). Interestingly, alterations in similar miRNA modules produce distinct phenotypic changes in gynoecium and fruits of Arabidopsis and tomato (Xing et al., 2013; Silva et al., 2014). Understanding what pathways are directly and/or indirectly regulated by similar miRNA modules in different species, and how they influence distinct fruit morphologies, will shed light on important evolutionary aspects of fruit 
development. In the next sections, we discussed examples in the literature concerning the roles of miRNA modules in early events of fruit development mostly in tomato and Arabidopsis.

\subsubsection{The miR164 module controls carpel development and leaf margin serration through similar mechanisms}

MiRNA-associated pathways control many aspects of plant development. Some miRNA-targeted transcriptional regulators that had their roles previously associated with vegetative development, such as leaf development, had similar functions later elucidated in carpel development. For instance, Arabidopsis miR164-targeted CUP-SHAPED COTYLEDON1 and 2 (CUC1 and CUC2) - which belong to the NAC transcription factor family - regulate organ boundary during the separation between organ primordia and meristem, and control leaf margin serration (Laufs et al., 2004; Nikovics et al., 2006; Peaucelle et al., 2007; Hasson et al., 2011; Vialette-Guiraud et al., 2016). Earlier studies showed that CUC1 and CUC2 operate during the initial phase of organ initiation inhibiting cell growth in meristem-organ and organ-organ boundaries, facilitating the separation between adjacent vegetative and reproductive organs (Laufs et al., 2004; Mallory et al., 2004). In this process, miR164 defines boundary domains by restricting the expression of CUC1 and CUC2 (the miR164 module), and proper miR164 dosage and/or expression localization is required for organ separation. The miR164 module also operates further in organ development, when organ shape is being determined (Nikovics et al., 2006). In the margins of leaf primordia, CUC2 and MIR164 $A$ are spatially and temporally coexpressed, and the balance between their expression controls the degree of Arabidopsis leaf margin serration (Nikovics et al., 2006). This module operates similarly in the regulation of leaf complexity in tomato, in which the CUC2 ortholog miR164-targeted GOBLET (GOB) plays similar roles during boundary establishment leading to leaflet separation. Interestingly, the regulation of compound leaf development by the miR164 module is conserved in Aquilegia caerulea, Solanum tuberosum, Cardamine birsuta, and Pisum sativum (Blein et al., 2008).

Like its function in leaf development, the miR164 module is also expressed in the margins of carpel primordium during Arabidopsis gynoecium development, and it determines important morphological characteristics of the mature fruit (Ishida et al., 2000; Sieber et al., 2007; Nahar et al., 2012; Kamiuchi et al., 2014; Vialette-Guiraud et al., 2016). Arabidopsis gynoecium is formed by two carpels that are already initiated as two fused structures, except by the apical margins, which are fused later to form style and stigma (Sessions and Zambryski, 1995; Nahar et al., 2012). During early gynoecia development, the meristematic tissue called Carpel Margin 
Meristem (CMM) is originated in the margins of each carpel primordia and is responsible for producing the ovules, the ovary septum, the transmitting tract, and promoting fusion between the apical carpel margins (Alvarez and Smyth, 1999; Nahar et al., 2012; Vialette-Guiraud et al., 2016). Earlier studies showed that CUC1 and CUC2 expression is required for the activation of the KNOX type-I gene SHOOT MERISTEMLESS (STM) in different developmental contexts, such as the formation of shoot apical meristem during embryo development and leaf serration in Arabidopsis (Takada et al., 2001). In such processes, STM expression is required to establish and maintain meristematic tissues. The same mechanism seems to operate in the establishment and maintenance of CMMs during carpel development in Arabidopsis (Kamiuchi et al., 2014). Most cuc1 cuc2 double mutants failed to form CMM, producing mature gynoecia with drastically reduced or complete loss of ovules and septum. Arabidopsis plants expressing miR164-resistant versions of CUC1 and CUC2 showed expanded domain of STM expression, resulting in carpel primordia with altered size and number of CMM, of which most initiated in altered positions. These plants produce mature fruits with internal filamentous structures (Kamiuchi et al., 2014). When not regulated by miR164, CUC1/2 expression is less precise and can expand out of the boundary strips, resulting in incorrect CMM positioning, which leads to carpel and fruit developmental aberrations.

SPATULA (SPT) encodes a basic helix-loop-helix (bHLH) transcription factor, and Arabidopsis loss-of-function spt mutants produce ovaries with split or incomplete fused carpels and defective CMM-derived tissues (Heisler et al., 2001; Nahar et al., 2012). cuc1;cuc2 mutations partially suppress the split carpel phenotype of $s p t$ mutant, indicating that congenital carpel fusion depends on SPT-based down-regulation of CUC1 and CUC2. Thus, the coordinated interaction among SPT, CUC1, and CUC2 regulates Arabidopsis ovule and septum development during the progression of fruit growth (Nahar et al., 2012). It was recently shown that SPT enables cytokinin signalling, which provides meristematic properties to CMM. SPT seems to play a role in the interaction between auxin and cytokinin pathways, as SPT induces ARABIDOPSIS RESPONSE REGULATOR 1 (ARR1) directly. SPT and $A R R 1$ induce the expression of the auxin transporter PIN-FORMED 3 (PIN3) and the auxin biosynthesis gene TRYPTOPHAN AMINOTRANSFERASE OF ARABIDOPSIS 1 (TAA1, Reyes-Olalde et al., 2017).

The role of the tomato CUC2 homolog GOB was studied in detail during leaf development and complexity, although little is known about the function of $G O B$ in reproductive development. Loss-of-function GOB mutant (gob-3) produces fruits with fewer locules, whereas gain-of-function $G O B$ mutant (which contains a miR164-resistant version of GOB, the Gob-4d) displays fruits with extra carpels and increased number of locules (Berger et al., 2009). Since leaf 
complexity was the main objective of this work, no mechanism was proposed of how the miR164 node (miR164-targeted GOB) controls locule number in tomato fruits. On the other hand, tomato miR164-targeted NO APICAL MERISTEM 2 (SINAM2), another member of the NAC transcription factor family, was shown to have an important role in organ boundary maintenance during floral development (Hendelman et al., 2013). Unlike GOB, SINAM2 is not expressed in boundaries between floral meristem and organ primordia, as SINAM2 expression was not detected before carpel fusion in flower buds. Data thus far suggest that $G O B$ functions during the formation of the boundaries, being expressed at earlier stages of organ primordia development, whereas SINAM2 is expressed at later stages of floral whorl development, being responsible for the maintenance of the boundaries established by GOB (Hendelman et al., 2013). Plants overexpressing mSINAM2 (a miR164-resistant version of SINAM2) produced gynoecia with shorter stamen and styles and wide pistil, the latter likely due to the extra carpel formation. Although weaker, mSINAM2 phenotypes were similar to Gob $4 d$ phenotypes, which is consistent with the proposed SINAM2 role in boundary maintenance, but not boundary formation (Berger et al., 2009; Hendelman et al., 2013). In summary, the functions of the miR164 module in Arabidopsis and tomato gynoecium patterning illustrates the crucial importance of boundary formation and maintenance during fruit development. Proper function of the miR164 module is essential for the establishment and maintenance of gynoecium development, not only in syncarpous species such as Arabidopsis and tomato, but also in monocarpous species like Medicago trunculata (Berger et al., 2009; Vialette-Guiraud et al., 2016).

\subsubsection{The role of $\mathrm{miR} 156 / \mathrm{miR} 157$ in carpel and fruit development}

MiR156 targets members of the SQUAMOSA PROMOTER BINDING PROTEINLIKE (SBP/SPL) transcription factor family. In Arabidopsis and tomato, 11 out of 17 SBP/SPLs harbor the miR156 recognition site (Salinas et al., 2012; Preston and Hileman, 2013). The miR156 module (miR156-targeted SBP/SPLs) defines the evolutionary conserved age-dependent floral pathway in several plants, including tomato (Silva et al., 2018). Interestingly, the miR156 module has been proposed as a main target for crop improvement, aiming to enhance agronomic traits such as the timing of vegetative and reproductive phase change, leaf development, tillering/branching, panicle/tassel architecture, fruit development and fertility (Wang et al., 2015).

In terms of gynoecium and fruit development, it was demonstrated that Arabidopsis SPL8 (which is not targeted by miR156) acts redundantly with miR156-targeted SPLs in the control of carpel development (Xing et al., 2013). Transgenic plants overexpressing miR156 
(p35S::MIR156b) produce flowers with reduced ovary size but unaffected structure, while ovaries of spl8-1 mutant show a slight reduction in size and resembles wild-type (W'T). Conversely, the double mutant $p 35$ S::MIR156b spl8-1 show extremely modified gynoecia. The gynoecium shape of p35S:::MIR156b spl8-1 is completely altered, displaying an enlarged upper region and a narrower basal region, abnormal septum development, and absence of transmitting tissue to support pollen tube growth into the ovary (Xing et al., 2013). Considering that SPL8 and the miR156-targeted SPLs 2, 6, 10, 11 and 13 are expressed in overlapping domains during gynoecium development, this data supports the idea that they have partly redundant roles in the patterning of the gynoecium and fruit development. Furthermore, seed production decreased about $60 \%$ in p35S::MIR156b plants in comparison with WT and spl8-1 (which show unaltered seed production), whereas $p 35 S:: M I R 156 b$ spl8-1 produces approximately $96 \%$ less seeds than WT (Xing et al., 2013). Together, these data indicate that the function of at least one of these SPLs is crucial for proper gynoecia development. Another study showed that Arabidopsis squint (sqn) mutants contain loss-of-function alleles for cyclophylin40 (CyP40), which increases the activity of miR156 by promoting $A G O 1$ activity. sqn plants showed elevated expression of miR156-targeted SPLs and produce siliques with increased carpel number (Smith et al., 2009).

Interestingly, the miR156 module may function by different mechanisms or have different roles in dry fruit and fleshy fruit-bearing species. As mentioned above, ovaries of Arabidopsis p35S:MIR156b plants do not present extra carpels or undetermined growth (Xing et al., 2013). On the other hand, the overexpression of miR156 ( $\not 35$ S:MIR156b) in tomato plants led to the production of extremely modified ovaries formed by multiple fused extra carpels and undifferentiated tissue inside the post-anthesis ovaries (Silva et al., 2014). After fruit set, the undifferentiated tissue inside the ovaries of $p 35 S:$ MIR156b plants continues to grow, forming fruit-like structures growing from the stylar end of the fruits. Furthermore, mature fruits show increased number of locules due to the presence of extra carpels in the ovary (Figure 1; Silva et al., 2014). Floral identity genes like FUL1/TDR4, FALSIFLORA (FA, Arabidopsis LEAFY ortholog; Lozano et al., 2009) and MACROCALLYX (MC, Arabidopsis APETALA1 ortholog; Lozano et al., 2009) were strongly down-regulated in tomato $p 355: M I R 156 b$ ovaries (Silva et al., 2014). Arabidopsis FUL, AP1 and LFY are direct targets of SPL3 (Yamaguchi et al., 2009), although it is still unknown whether their tomato orthologs are direct targets of SISBP3. Interestingly, the CUC2 and STM orthologs GOB and TKN2, respectively, are up-regulated in tomato p35S:MIR156b ovaries. MiR164-targeted GOB and TKN2 are associated with leaf complexity in tomato but both can also regulate the number of locules per fruit (Parnis et al., 1997; Berger et al., 2009). This finding indicates a link between miR156 and miR164 modules and 
suggests that tomato miR156 module controls boundary formation and establishment as well as locule number through $G O B$ and perhaps other NAC domain-containing genes (such as SINAM2). As expected, tomato plants overexpressing miR164 lead to GOB down-regulation and the production of fruits with normal shape but reduced locule number (Silva et al., 2014).

Most plant genomes also contain miR157, a miR156 closely related miRNA which differs from miR156 by three nucleotides (Reinhart et al., 2002). MiR157 overexpression in Arabidopsis generates plants phenotypically similar to miR156 overexpressors, but miR157 specific functions are still unknown (He et al., 2018). MiR157 seems to be more abundant but less effective on SBP/SPL repression, perhaps because it is less efficiently loaded onto AGO1 (He et al., 2018). Transgenic cotton plants overexpressing miR157 produced smaller gynoecium, with less ovules per ovary and decreased seed production in comparison with WT (Liu et al., 2017). These plants showed reduced expression of two MADS-box transcription factors (orthologs of AtAGL6 and STDR8). In addition, auxin response was attenuated in ovaries of miR157overexpressing cotton plants. It is possible that miR156 and miR157 modules regulate gynoecium development by overlapping but also specific mechanisms, although additional studies are needed to unravel miR157 specific functions in reproductive development.

\subsection{3. miR396 module regulate CMM meristematic competence and pluripotency during gynoecium development}

The GROWTH-REGULATING FACTORs (GRFs) belong to a plant-specific transcription factor family that has nine members in Arabidopsis, seven of which (GRF1, GRF2, GRF3, GRF4, GRF7, GRF8, and GRF9) are targeted by miR396, representing the miR396 module (Lee et al., 2014; Liang et al., 2014; Lee et al., 2017). MiR396 module regulates several developmental processes, such as leaf development, floral development, and root cell reprograming during nematode infection (Lee et al., 2009; Hewezi et al., 2012).

Arabidopsis plants overexpressing miR396 (p35S:MIR396a) show gynoecium developmental defects such as gynoecia formed by only one carpel and siliques (dry fruits) producing a reduced number of seeds (Liang et al., 2014; Lee et al., 2017). Arabidopsis GRFs interact physically in the nucleus with the transcriptional co-activators GRF-INTERACTING FACTOR1, 2 and 3 (GIF1, GIF2 and GIF3) (Liang et al., 2014). Because GIF-GRF complexes are crucial for meristematic competency and pluripotency of CMM cells (Lee et al., 2017), high miR396 levels may lead to low GRFs available to form these heterodimers, hence CMM loses its meristematic competence and pluripotency over time (Liang et al., 2014; Lee et al., 2017). Single 
GRF loss-of-function mutants produce WT-like siliques, whereas gif1 single mutant produces normal pistil and siliques but with reduced size. Siliques of the double transgenics $p 35$ S:MIR396a ,p35S:mGRF7 and p35S:MIR396a ; 355 S:mGRF9 (both expressing miR396-resistant versions of GRF7 and 9 transcripts, respectively) can recover WT silique phenotypes, indicating that at least miR396-targeted GRF7 and 9 have roles in fruit development (Liang et al., 2014). The phenotypes of gynoecium and siliques of the triple mutant gif1 gif2 gif3 phenocopy those of the double mutant p35S:MIR396a grf5 (GRF5 is not targeted by miR396), producing extremely short and almost sterile siliques, generally lacking valves, whereas some GRF triple mutants (e.g. grf1/grf2/grf3 and $g r f 7 / g r f 8 / g r f 9)$ present WT-like siliques (Liang et al., 2014). The triple mutant grf1 grf3 grf5 show single-valve gynoecia and slight defects on floral organ separation and number, but these defects were strongly enhanced by the addition of grf 2 mutation to this background (generating the quadruple mutant grflgrf2 grf3 grf5). Together, these findings indicate that GRFs act redundantly to modulate Arabidopsis gynoecium patterning and fruit development.

The mechanisms by which GRF-GIF dimers promote CMM meristematic capacity in Arabidopsis gynoecium were not well elucidated, but available data suggest that they may be associated with polar auxin transport (PAT) (Lee et al., 2017). Arabidopsis PAT mutants (pinformed 1 and pid) and some auxin biosynthesis mutants (yuc1, yuct and wei8 tar2) produce gynoecia phenotypes identical to gif p35S:MIR396a plants and grf multiple mutants. The addition of gif mutations to a pid-3 mutant (a PINOID mutant with weak developmental defects) or treatment of gif mutants with N-1-Naphthylphthalamic Acid (NPA, an auxin polar transport inhibitor) synergistically enhance gynoecium developmental defects of pid-3 or NPA-treated W'T plants (Lee et al., 2017). These findings indicate an interplay between miR396, GRF-INTERACTING FACTORs and auxin during gynoecium patterning.

Unlike Arabidopsis, the possible role of the miR396 module in tomato fruit development has not been described in detail. The only study in tomato thus far showed that miR396 downregulation (or GRF de-regulation) seems not to affect CMM formation but rather it leads to a significant increase in fruit size (Cao et al., 2016). This is consistent with the main role of GRFs in modulating cell proliferation and cell expansion in several developmental contexts (Lee et al., 2009). Since neither fruit shape nor ripening was altered in the transgenic tomato plants downregulating miR396 (Cao et al., 2016), the authors proposed that these plants might provide a new way to enhance tomato fruit yield. 


\subsection{4. microRNA160 module controls carpel development by modulating auxin responses}

Some microRNAs, such as miR160, are crucial for auxin signaling during several developmental processes. MiR160, which targets the AUXIN RESPONSE FACTORS ARF10, 16 and 17 (Hendelman et al., 2012; Damodharan et al., 2016; Li et al, 2016), is another example of a miRNA module that apparently has different roles in the regulation of dry and fleshy fruit development.

The Arabidopsis floral organs in carpels (foc) mutant contains a $D$ s transposon insertion in the 3 'regulatory region of the MIR160a gene, which disrupts its native expression pattern, leading to the accumulation of $A R F 10,16$ and 17 and low auxin responses in various organs (Liu et al., 2010). These regulatory disruptions lead to abnormal embryo, seed, and flower development. foc plants show some degree of indeterminacy during gynoecium patterning, which is observed by the production of floral organs inside the siliques and sometimes whole inflorescences emerging from siliques. Furthermore, foc mutant produces abnormal seeds and viviparous seedlings. It was also shown that 3 'regulatory region bears three putative auxin-responsive elements (AuxRE) and MIR160a expression is positively regulated by auxin. Thus, the disruption of this regulatory region impairs the induction of MIR160a expression by auxin, impacting fruit development (Liu et al., 2010).

The miR160 module (miR160 and their targets) seems also to have an important, but different, role in tomato fruit development. Transgenic tomato plants (STTM160-expressing plants) with knocked-down miR160 expression generated by the Short Tandem Target Mimic (STTM) approach (Teotia and Tang, 2017) produce ovaries with elongated morphology and thinning of the placenta, which developed into fruits with abnormal pear-shaped fruit morphology. These changes were associated with miR160 depletion and concomitant deregulation of SLARF1OB and SLARF17, and mostly SLARF10A in STTM160-expressing plants (Damodharan et al., 2016). Nevertheless, unlike Arabidopsis foc mutant, no indeterminacy was observed in gynoecia of STTM160-expressing tomato plants. Such discrepancy between phenotypes of tomato and Arabidopsis miR160 loss-of-function plants may be due to the fact that SLARF16 is not de-regulated in STTM160-expressing tomato plants, despite the miR160 legitimate site observed in SIARF16 (Damodharan et al., 2016).

MiR160-guided cleavage of some ARFs is also needed for proper leaf development in tomato and Arabidopsis. Interestingly, STTM160 tomato plants and 5mARF17 (plants expressing a miR160-resistant version of ARF17) Arabidopsis plants showed similar leaf 
phenotype, which is reduced leaf blade and strongly lobbed leaflet/leaf margins (Mallory et al., 2005; Damodharan et al., 2016).

\subsection{5. miR172 limits the growth-repressing activity of APETALA2-like genes during fruit expansion}

All microRNA modules discussed so far are mostly associated with very early stages of carpel development, such as patterning and differentiation, and the proper control of these stages have great impact on mature fruit morphology and fertility. On the other hand, the miR172 module seems to control not only fruit patterning, but also fruit growth, which comprises a developmental stage after pollination, when the ovary is fully developed. In Arabidopsis, the miR172 module comprises the microRNA172 and its targets (APETALA2-LIKE (AP2-like) transcription factors): APETALA2 (AP2), TARGET OF EAT1, 2 and 3 (TOE1, TOE2, and TOE3), SCHLAFMUTZE (SMZ), and SCHNARCHZAPFEN (SNZ) (Wu et al., 2010). Interestingly, pioneer studies showed that miR172 can guide not only AP2-like transcript degradation but also its translational repression (Chen, 2004).

Arabidopsis fruit undergoes dramatic increase in fruit size after fertilization, and different tissues grow at different rates (for review please see Ferrándiz et al., 2010). MiR172 module seems to be crucial to specify which regions of the carpel will go through dramatic expansion and which region will arrest fruit growth. AP2 encodes an AP2/EREBP transcriptional repressor, which was shown to repress valve margin and replum growth post-fertilization by repressing the expression of genes that confer identity to valve margin (INDHEISCENT and SHATTERPROOF) and replum (BREVIPEDICELLUS and REPLUMLESS) (Ripoll et al., 2011). In this context, AP2 prevents replum overgrowth and overproliferation of the layer of lignified cells (LL) (which are associated with fruit dehiscence; Rajani and Sundaresan et al., 2001; Liljegren et al., 2004) in the valve margin. Consistent with this, ap2 mutants produce siliques with oversized replum and slightly delayed dehiscence due to increased number and size of LL (Ripoll et al., 2011). Nevertheless, after pollination the valves undergo a conspicuous cell expansion stage, increasing dramatically fruit size. This pollination-dependent valve growth was shown to be blocked in plants with decreased miR172 activity - via target mimicry (MIM172) approach (Franco-Zorrilla et al., 2007) - and in plants expressing a miR172-resistant AP2 version, resulting in smaller fruits (Ripoll et al., 2015). For proper valve expansion, AP2 and TOE3 activities must be inhibited by miR172 only in the valves. The MAD-box transcription factor FRUITFULL (FUL) displays similar expression pattern as miR172, being expressed in the valves, and ful 
mutants resemble MIM172 plants, presenting arrested growth phenotype in the valves. Furthermore, analysis of different degrees of homo and heterozygosity of ARF6 and ARF8 mutant alleles arfo and arf 8 in double mutants show that fruit valve expansion decreases with the increasing $A R F$ mutant allelic dosage. Valve growth is even more limited when arf $6 / 8$ are introduced in $f u l$, and arfo arf8 ful triple mutants produce siliques with extremely impaired growth. FUL, $A R F 6$, and $A R F 8$ are expressed only in the valves (except valve margins), where they form protein complexes that bind to the MIR172C promoter and activate its expression. AP2 and TOE3 are expressed in the whole carpel, but miR172 induction in the valves restricts AP2 activity to the valve margins and replum, allowing it to repress cell elongation in these locations but not in the valves. Through this mechanism, miR172 fine-tunes fruit patterning and growth by restricting the activity of AP2-like genes to certain locations within the fruit (Ripoll et al., 2015). Considering that miR167 negatively regulates ARF6 and ARF8 (Wu et al., 2006), it will be interesting to determine whether this miRNA participates in this mechanism by specifying ARF6/8 expression pattern.

Although high levels of miR172 have a positive effect on Arabidopsis fruit growth (Ripoll et al., 2015), this is not always the case for other species. For instance, over-expression of a MIR172 gene has a negative influence on fruit growth in apple (Malus domestica), resulting in a dramatic reduction in fruit size (Yao et al., 2016). Unlike Arabidopsis and tomato fruits, which are both derived from ovaries, apple fruits are mostly derived from the hypanthium that is hypothesized to consist of the fused bases of the sepals, petals and stamens (Pratt, 1988). Interestingly, over-expression of the same MIR172 gene in tomato results in carpel-only flowers which developed into parthenocarpic fruits (Yao et al., 2016). These examples nicely illustrate that the influence of a particular miRNA module on fruit growth depends on the fruit type and plant species.

\subsection{MicroRNA-controlled pathways modulating ovule and seed development during fruit growth}

The ovule is the female sexual organ in higher plants and a strict control of ovule development is crucial for plant reproductive success. Ovule is required to enclose the female gametophytes and, more importantly, it is from the fertilized ovules that seeds arise. Ovule structures are conserved in most plants, and comprise the embryo sac, the nucellus, the integument (which originates the seed coat) and the funiculus, which makes the connection between the ovule and placenta. Ovule and seed development are under control of genetic (e.g., transcription factors, non-coding RNAs), physiological (hormones) and epigenetic factors (i.e. 
chromatin remodelling and DNA methylation) (Skinner et al., 2004; Kelley and Gasser, 2009; Yamaguchi et al., 2013; Cucinotta et al., 2014). In this part of the review, we will discuss the findings of how some small RNAs modules act to modulate ovule and seed development, which are crucial developmental processes that take place during fruit development and ripening.

It was recently shown by our research group that the miR159 module is crucial for ovule and seed development in tomato (Silva et al., 2017). The miR159 module comprises the microRNA159 and its targets, S/GAMYB1 and S/GAMYB2, which belong to the R2R3 MYB domain transcription factor family. GAMYB-like genes are regulated by gibberellin and by the microRNA159 family in different tissues and developmental contexts (Gubler et al., 1995; Tsuji et al., 2006; Alonso-Peral et al., 2010). MiR159 and its targets are expressed early during tomato placenta and ovule development, which suggest that the miR159 module may be involved in the initial steps of ovule development. Likewise, the overexpression of SIMIR159 (p35S::SIMIR159) disrupts ovule development and induces obligatory parthenocarpy. Such phenotype is more severe than what is shown in AtMIR159a-overexpressing Arabidopsis plants, which generates fertile siliques when pollinated with W'T pollen (Achard et al., 2004). Tomato, transgenic plants harboring the $p 35$ S::SIMIR159 construct displays defects in the establishment of the embryo sac, which may be due to the observed lower expression of AINTEGUMENTA-like genes (Silva et al., 2017). AINTEGUMENTA (ANT) gene is an APETALA2-like transcription factor required for ovule and integument initiation (Elliott et al., 1996). Although tomato lacks known $A N T$ mutants, it was shown in rice that $A N T$ was also strongly repressed in gamyb mutants displaying ovule developmental defects (Tsuji et al., 2006). MiR159 module interacts with tomato AINTEGUMENTA-like genes to drive developmental progression of ovules and, thus, modulates tomato fruit set. Moreover, our work showed that miR159 module interacts with the miR167 module. Down-regulation of miR167 and concomitant SLARF8 de-regulation in p35S::MIR159 plants may be also responsible for the arrested ovule development (Silva et al., 2017), illustrating the link between the miR159 module and auxin during fruit set.

Parthenocarpy, the developmental process in which fruits develop in the absence of fertilization (Varoquaux et al., 2000), can be easily induced in grapevine (Vitis vinifera) by exogenous gibberellin (GA) application (Wang et al., 2018). These authors show that VvmiR159c and its target $V v G A M Y B$ are dynamically and opposing expressed during flowering and fruit set. GA treatment is capable of inducing $V v m i R 159 c$ and, consequently, down-regulating $V v G A M Y B$ in reprouctive organs. These observations led the authors to suggest that the miR159 module is associated with GA-induced parthenocarpy in grapevine (Wang et al., 2018), similarly to what we have discovered in tomato (Silva et al., 2017). 
The use of high-throughput sequencing approaches also provided evidences of the activity of miRNA modules during ovule development. In cotton (Gossypium birsutum), small RNAs profiles of developing ovaries showed distribution of several small RNA signatures, including microRNAs (Abdurakhmonov et al., 2008). Several conserved microRNA families were identified in cotton ovules, including miR156/157, miR159, miR164, miR168, and miR395. These results are important to provide initial information for future functional experiments. In addition, several predicted miRNA targets were validated via degradome sequencing (a modified version of 5'-Rapid Amplification of cDNA Ends that is combined with high-throughput, deep sequencing to detect transcript ends; Ma et al., 2015), reinforcing the idea that conserved miRNA modules may be important in ovule development of cotton (Xie et al., 2015).

MicroRNAs are also required for embryogenesis, which is a key developmental step for plants to establish the seed set. To complete its development, the embryo undergoes specific stages, which in Arabidopsis are defined by its morphology as globular, heart, torpedo, and walking stick stages (Jürgens, 2001). Such developmental stages are known to be regulated by transcription factors, small regulatory RNAs, signal transduction orchestrated by kinases, auxin gradients, and epigenetic mechanisms (i.e. DNA methylation, histone acetylation, among others). Thus, these regulatory pathways are key determinants of the fate of primordia cell lineages, and also drive inheritance that is programmed via mitosis at early stages of the embryo development (Willemsen and Scheres, 2004).

DICER-LIKE1 (DCL1) is a key enzyme for the pri-/pre-miRNA processing (Park et al. 2002; Reinhart et al. 2002; Kurihara and Watanabe 2004). Genome-wide transcriptional profiling of the Arabidopsis mutant dicer1 (dcl1) shed some light regarding the importance of microRNA modules during early embryo development. At the early globular stage, dcl1 embryo display about 50 miRNA targets de-repressed due to the lack of miRNA regulation. Some of these targets (usually transcription factors) are required for differentiation at later stages of embryogenesis (Nodine and Bartel 2010). In addition, in dcl1 embryos, miR156-targeted SPL10 and SPL11 are highly up-regulated, which suggest that the de-regulation of these transcription factors is at least in part responsible for the $d c l 1$ embryo abnormalities (morphological defects and arresting growth at the globular stage). Thus, one of the first roles of plant microRNAs is to repress its targets at early developmental stages to prevent precocious differentiation during embryogenesis (Nodine and Bartel 2010). This idea is further supported by the finding that Arabidopsis double mutant ago1/ago10 displays embryo lethality, probably due the highly activity of small RNAs targets (Lynn et al. 1999; Mallory et al., 2009). Argonaute (AGO) proteins are part of the RNA-induced 
silencing complex (RISC), and are required for the repression of microRNA targets (Rhoades et al. 2002; Zilberman et al., 2003).

MiRNA module may also affect seed development. MiR397 negatively regulates members of the Laccase family. MiR397-targeted Laccase4 is a member of the blue copper oxidase/ p-diphenol:dioxygen oxidoreductase family and participates in lignin biosynthesis. (Gavnholt and Larsen, 2002; Mayer and Staples, 2002). The miR397/Laccase4 module has been implicated in the control of the number of seeds and seed size. Overexpression of MIR397b in Arabidopsis leads to reduce lignin deposition. Interestingly, in terms of fruit development, transgenic plants with less lignin produce bigger siliques with more and enlarged seeds. Similar results are observed in transgenic rice plants overexpressing MIR397a and MIR397b, which are able to produce enlarged grains (Wang et al., 2014; Zhang et al., 2013). Such studies highlight that miR397-mediated development via regulating laccase genes might be a potential tool not only for engineering plant biomass production with less lignin, but also for manipulating plant seed yield.

\subsection{Noncoding RNAs in the regulation of fruit ripening}

In the first section of this review, we discussed the main microRNA modules involved in diverse aspects of early fruit development, which is summarized in Figure 2A. In this last section, we will discuss a few examples available in the literature that reinforce the fundamental roles of non-coding RNA-mediated regulation also in fruit ripening.

\subsubsection{Conserved and Solanaceae-specific miRNA modules control tomato fruit ripening}

Tomato plants bearing the dominant mutation Cnr (COLORLESS NONRIPENING) produce fruits with characteristics associated with impaired ripening, such as inhibited softening, yellow skin, and pericarp lacking pigments because of the arrested biosynthesis of ripeningrelated pigments (Thompson et al., 1999). Furthermore, mutant plants produce lower amounts of ethylene and exogenous ethylene application does not recover this phenotype. Positional cloning showed later that a SPL/SBP gene (called SISBP3/CNR) containing a potential miR156/157 binding site resides in the $C n r$ locus. $C n r$ is an epimutation caused by spontaneous heritable hypermethylation of cytosine residues of the SISBP3/CNR promoter, leading to SISBP3/CNR repression (Manning et al., 2006). Although the mechanism by which SISBP3/CNR controls fruit ripening remains unclear, recent data suggest that the MADS-box transcription factor 
RIPENING INHIBITOR (RIN) and CNR may be part of the same protein complex that induces the expression of ripening-related genes (Martel et al., 2011). RIN controls both ethylenedependent and independent ripening regulatory pathways, interacting directly with the promoter of many known genes associated with key ripening processes, such as ethylene biosynthesis, perception and signal transduction, cell wall metabolism, and carotenoid biosynthesis. Nevertheless, CNR is required for RIN promoter binding activity, as RIN does not interact with the promoters of ripening-related genes in the Cnr mutant (Fujisawa et al., 2013; Martel et al., 2011; Qin et al., 2012). Although CNR and RIN proteins do not interact, it is possible that these transcription factors are part of the same protein complex that modulates the expression of key ripening genes. Substantiating this hypothesis, rin and $C n r$ mutants have similar fruit phenotypes such as blocked ripening and impaired response to exogenous ethylene (Vrebalov et al., 2002; Martel et al., 2011).

Virus-induced gene silencing (VIGS)-based delivery of mature miR157 in tomato fruits reduced $C N R$ transcript accumulation and delayed ripening in the injected fruit areas (Chen et al., 2015). Degradome analyses indicate that miR156 cleaves CNR in different stages of fruit ripening (Karlova et al., 2013). Surprisingly, VIGS-based delivery of miR156 does not produce any alteration in fruit ripening until the breaker stage, and these fruits show early softening (Chen et al., 2015). These observations suggest that the miR156/miR157 module may be necessary for proper control of fruit ripening and that the closely-related miR156 and miR157 play different roles in the temporal control of the ripening-associated processes.

Tomato miR172-targeted AP2a appears to have complex functions in the control of diverse ripening-related processes, regulating mostly genes associated with ethylene biosynthesis and signaling (Karlova et al., 2011). AP2a silencing through RNAi leads to the production of fruits that ripe, but never turn from orange to red, showing altered levels of various carotenoids and increased chlorophyll levels, although they produce high levels of ethylene. AP2a seems to act downstream to RIN and $C N R$, as its expression is negatively regulated in rin and $C n r$ mutants and CNR binds to $A P 2 a$ promoter. Thus, CNR induces $A P 2 a$ expression directly, although $A P 2 a$ represses CNR expression in a negative feedback loop (Karlova et al., 2011). Taken together, the evidences in tomato thus far indicate that both miR156/miR157 and miR172 modules and the interaction between their targets (CNR and $A P 2 a)$ are important to proper fruit ripening. In fact, degradome analysis showed that levels of the CNR and AP2a miRNA-guided cleavage products vary among different ripening stages, showing peak accumulation of cleavage transcripts during breaker stage, which is also the peak of ethylene production (Karlova et al., 2013). It will be 
interesting to determine whether these miRNAs have specific roles in fine-tuning spatially and/or temporally the expression of their targets during fruit ripening.

Recently, a novel miRNA identified as Solanaceae-specific was implicated in regulating ethylene signaling and hence fruit ripening in tomato (Wang et al., 2018). The microRNA miR1917 targets three splicing variants of the CONSTITUTIVE TRIPLE RESPONSE 4 (SlCTR4, homolog of Arabidopsis CTR1), an ethylene signaling repressor that interacts with ethylene receptors (Wang et al., 2018). Tomato plants overexpressing the miR1917 (p355::MIR1917) display higher levels of ethylene signaling, leading to enhanced ethylene production. These plants also have increased ethylene responses in the absence of ethylene, including accelerated pedicel abscission and fruit ripening (Wang et al., 2018). The complementary expression pattern of miR1917 and the splicing variants S/CTR4sv3 observed in the pedicel abscission zone by in situ hybridization suggests that miR1917 restricts the expression of its targets to the vascular bundle and surrounding cells during pedicel abscission. Thus, miR1917 and its targets represent a novel miRNA module belonging to the intricate ethyleneassociated signaling network.

\subsubsection{New evidences of the role of long non-coding RNAs (IncRNAs) in fruit ripening}

Long non-coding RNAs are broadly present in plant, animal and fungi transcriptomes and emerging evidences show that they play key roles in diverse developmental processes. They are RNAs longer than 200 nt originated from transcription of intergenic regions, introns or antisense coding sequences and do not have any detecting coding potential (Chekanova et al., 2007; Chekanova, 2015; Fatica and Bozzoni, 2014; Kapranov et al., 2007). LncRNAs may modulate gene expression by multiple mechanisms that were extensively reviewed in Chekanova (2015). Although the knowledge of the regulatory roles of lncRNAs in plants is still limited, lncRNAs have been associated with the control of flowering time, male sterility, seedling morphogenesis and, more recently, fruit ripening (Berry and Dean, 2015; Ding et al., 2012; Li et al., 2018; Wang et al., 2014).

RNA-seq analyses comparing transcriptomes of tomato cv Ailsa Craig and rin fruits identified over 3000 tomato lncRNAs, several of which were differentially expressed in rin (Zhu et al., 2015). In the same study, two lncRNAs (lncRNA1459 and lncRNA1840) strongly downregulated in rin were chosen for VIGS-based silencing assays in fruits. Silencing of both lncRNAs produced non-ripening sections in the injected areas of the fruit, similarly to the effect observed 
in VIGS-based silencing of RIN. To better understand the functional role of lncRNA1459, which is a sense intergenic lncRNA, Li et al. (2018) generated loss-of-function mutants for lncRNA1459 using clustered regularly interspaced short palindromic repeats (CRISPR)/-associated protein 9 (Cas9)-induced genome editing technology (Feng et al., 2013; Doudna and Charpentier, 2014). Mutant fruits display delayed ripening phenotype associated with repressed ethylene and carotenoid biosynthesis, as well as down-regulation of ripening-associated genes.

In addition to tomato, lncRNAs involved in fruit ripening have been identified and studied in few other species. Sea buckthorn (Hippophae rhamnoides) is a plant for

land reclamation, and its berry-type fruits have high nutritional value due to the significant amounts of natural anti-oxidants including ascorbic acid, tocopherols, carotenoids, and flavonoids (Zakynthinos et al., 2016). By using high throughput RNA sequencing, Zhang et al. (2018) identified over 9000 lncRNAs expressed in distinct sea buckthorn fruit developmental stages, from mature green to red-ripe. Interesting, the authors identified two lncRNAs (LNC1 and LNC2) that may function as target mimics of miR156 and miR828 during fruit ripening, therefore indirectly affecting the expression of these miRNA targets, SPL9 and MYB114, respectively. By modulating SPL9 and MYB114 expression, LNC1 and LNC2 seem to control the biosynthesis of anthocyanin during fruit ripening (Zhang et al., 2018).

Despite the examples given above, the functions of the majority of ripening-associated lncRNAs are still unclear. More functional studies are needed to confirm the function of lncRNAs and their possible target genes. On possibility to be further explored is that lncRNAs can interact with microRNAs to modulate gene expression level (Gorospe et al., 2014), thus combining the "power" of two ncRNAs to modulate fruit ripening (Figure 2B).

\subsection{Conclusion}

During plant development, multiple microRNA modules are required to control meristem identity, leaf margin serration, polarity, complexity, root development, and flowering time. As summarized here, miRNA modules have key roles in fruit development, ranging from carpel establishment and patterning to fruit ripening. Disruption of miRNA transcription or processing frequently generate pleotropic consequences for the plant. Indeed, their activity are essential for plants to complete their life cycle, since they are active from seed to flower production. Interestingly, evolution of miRNA modules brought about adaptative advantages to plants by using similar pathways to orchestrate different developmental processes. A good example presented here is the miR164 module, which is required for proper leaf and carpel/fruit development, corroborating the hypothesis of the evolutionary origin of carpel as modified 
leaves. It is interesting to consider that evolution has also hijacked similar microRNAs modules to control unrelated developmental programs such as the role of the miR156 module in flowering time and fruit development and ripening. In addition, due to their multiple roles in plant development, microRNA modules may also provide promising molecular tools to be explored in an agricultural context. Therefore, the better understanding of the mechanisms that control miRNA and target expression and their spatiotemporal regulatory roles could be an outstanding step towards the application of microRNA-targeted regulation of important fruit traits, including size, shape, seed production, and ripening. For instance, the use of novel CRISPR/Cas9-based technologies (Li et al., 2018) might allow subtle changes in miRNA target gene expression which have a potential to quantitative modify fruit traits. Additionally, it would be interesting to investigate whether there are more specific microRNAs modules (e.g., Solanaceae-specific microRNA mentioned in this review) in others crops that might be associated with fruit quality traits. Although there are open questions of how microRNA modules function during fruit development, lncRNA-associated pathways are probably one of the less understood so far, involving multiple and complex origins and modes of action. As mentioned in this review, microRNAs and lncRNAs act during fruit ripening, and they can interact during this process. In addition, since they may have overlapping functions during ripening, it would be interesting to investigate whether these two classes of non-coding RNAs interact in early steps of carpel development and fruit patterning as well. The identification of additional lncRNAs and miRNAs and the understanding of how they interact with each other to control fruit development and ripening would be an important step towards the improvement of fruit production. The use of next generation sequencing technologies combined with functional genomics may help to achieve this goal.

Author contributions: EMS, JPOC, and FTSN conceived the review. EMS and JPOC wrote the review. FTSN helped writing and correcting the final version of the review.

Acknowledgements: We thank Dr. Nogueira's lab members for helpful discussions. EMS and JPOC were the recipient of a State of Sao Paulo Research Foundation (FAPESP) fellowships nos. 13/02469-6 and 16/05073-4, respectively. This work was supported by FAPESP (grant no. 15/17892-7).

Conflict of Interest: the authors declare that they have no conflict of interest. 
Figures
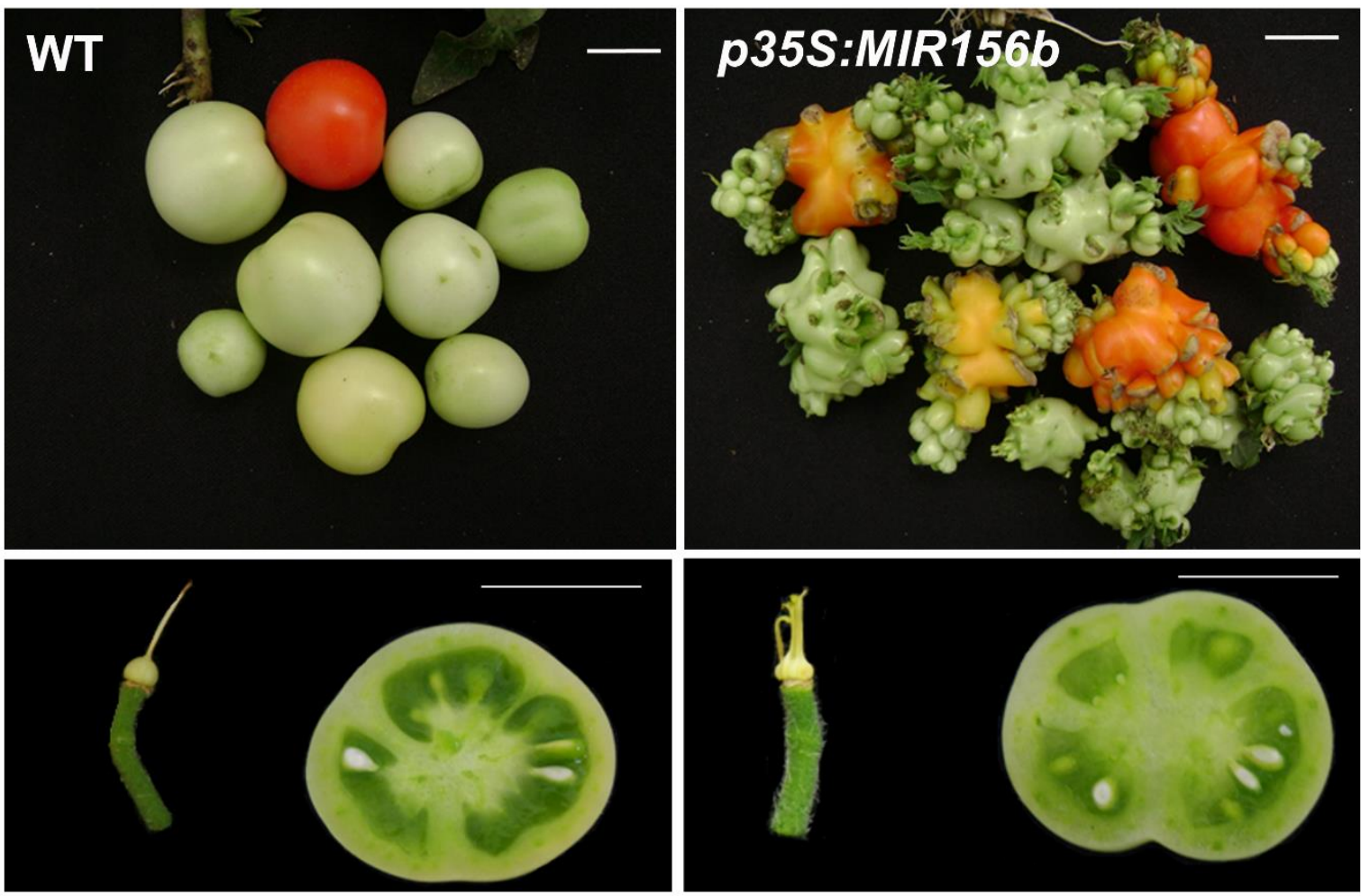

Figure 1. MIR156 overexpression alters tomato fruit determinacy and locule number. Upper panels: wild-type (WT) fruits and undetermined $p 355:: M I R 156 b$ fruits. Low panels: three-locular WT fruits and four-locular $p 355:: M I R 156 b$ fruits. Bars: $1 \mathrm{~cm}$. 



Figure 2. Non-coding RNA networks associated with carpel patterning and fruit ripening. (A) Summary of miRNA modules that control early (e.g., CMM establishment and maintenance) and late aspects of Arabidopsis fruit development. MiR396 and miR164 modules have important regulatory roles in CMM maintenance. MIR172C is induced by AUXIN RESPONSE FATORS ARF6/8 and FRUITFULL (FUL) specifically in the valves, and this specificity is necessary for proper fruit growth after pollination. MiR172-guided APETALA2 (AP2) mRNA cleavage in the valves (but not valve margins) promotes valve growth due to the repression of $A P 2$ growth-blocking activity. Growth is blocked by AP2 in valve margins and replum, where miR172 is not expressed. 1 - stigma, 2 - style, 3 valve, 4 - Valve margin, 5 - replum, 6 - septum, 7 - ovule, 8 - carpel margin meristem (CMM). GRF, GROWTHREGULATING FACTOR; GIF, GRF-INTERACTING FACTOR; STM, SHOOT MERISTEMLESS. (B) Graphic shows the accumulation of miRNA-cleaved transcripts of CNR and AP2 through four stages of fruit development/ripening: 5 days after pollination (5 DAP), Mature green $(\mathrm{MG})$, Breaker $(\mathrm{Br})$ and Red ripe (RR) (adapted from Karlova et al., 2013). MRNA cleaved product accumulation occurs in the breaker stage, coinciding with an ethylene peak production. Lnc1840 and lnc1459: long non-coding RNAs. Black arrows in the transcriptional networks denote direct regulation, whereas grey arrows denote indirect regulation. Question mark denotes that is uncertain if CNR forms a complex with RIN. SICTR4, tomato CONSTITUTIVE TRIPLE RESPONSE 4. 


\section{REFERENCES}

Abdurakhmonov, I. Y., Devor, E. J., Buriev, Z. T., Huang, L., Makamov, A., Shermatov, S. E, et al. (2008). Small RNA regulation of ovule development in the cotton plant, G. hirsutum L. BMC Plant Biology. 8:93 doi: 10.1186/1471-2229-8-93

Achard, P., Herr, A., Baulcombe, D.C. and Harberd, N.P. 2004 Modulation of floral development by a gibberellin-regulated microRNA. Development, 131, 3357-3365 doi: 10.1242/dev.01206Aida, M., Ishida T., and Tasaka, M. (1999). Shoot apical meristem and cotyledon formation during Arabidopsis embryogenesis: interaction among the CUPSHAPED COTYLEDON and SHOOT MERISTEMLESS genes. Development (Cambridge, England) 126, 1563-70

Alonso-Cantabrana H., Ripoll J.J., Ochando I., Vera A., Ferrándiz C., Martínez-Laborda A. (2007). Common regulatory networks in leaf and fruit patterning revealed by mutations in the Arabidopsis ASYMMETRIC LEAVES1 gene. Development. 134, 2663-71. doi: 10.1242/dev.02864.

Alonso-Peral MM, Li J, Li Y, Allen RS, Schnippenkoetter W, Ohms S, White RG, Millar AA. (2010). The microRNA159-regulated GAMYB-like genes inhibit growth and promote programmed cell death in Arabidopsis. Plant Physiol 154, 757-771.

Alvarez, J.P., and Smyth, D.R.. (1999). CRABS CLAW and SPATULA, two Arabidopsis genes that control carpel development in parallel with AGAMOUS. Development. 126, 2377-86.

Aukerman, M.J., and Sakai, H. (2003). regulation of flowering time and floral organ identity by a microRNA and its APETALA2-like target genes. The Plant Cell. 15, 2730-41. doi:10.1105/tpc.016238.pression

Berger, Y, Harpaz-Saad S., Brand A., Melnik H., Sirding N., Alvarez J.P., et al. (2009). The NACdomain transcription factor GOBLET specifies leaflet boundaries in compound tomato leaves. development. 136, 823-32. doi:10.1242/dev.031625

Berry, S., and Dean, C. (2015). environmental perception and epigenetic memory: mechanistic insight through FLC. The Plant Journal. 83, 133-48. doi:10.1111/tpj.12869

Blein, T., Pulido A., Vialette-Guiraud A., Nikovics K., Morin H., Hay A., et al. (2008). a conserved molecular framework for compound leaf development. Science. 322, 1835-39. doi:10.1126/science.1166168. 
Borges, F., and Martienssen R.A.. (2015). the expanding world of small rnas in plants. Nature Reviews Molecular Cell Biology. 16, 727-41. doi:10.1038/nrm4085

Cao D, Wang J, Ju Z, Liu Q, Li S, Tian H, Fu D, Zhu H, Luo Y, Zhu B. (2016) Regulations on growth and development in tomato cotyledon, flower and fruit via destruction of miR396 with short tandem target mimic. Plant Sci. 2016 Jun;247:1-12. doi: 10.1016/j.plantsci.2016.02.012

Chekanova, J.A. 2015. Long non-coding RNAs and their functions in plants. Current Opinion in Plant Biology. 27, 207-16. doi:10.1016/j.pbi.2015.08.003

Chekanova, J.A., Gregory B.D., Reverdatto S.V, Chen H., Kumar R., Hooker T., et al. (2007). Genome-wide high-resolution mapping of exosome substrates reveals hidden features in the Arabidopsis transcriptome. Cell. 131, 1340-53. doi:10.1016/j.cell.2007.10.056

Chen, W., Kong J., Lai T., Manning K., Wu C., Wang Y., et al. (2015). Tuning LeSPL-CNR expression by SlymiR157 affects tomato fruit ripening. Scientific Reports. 5, 1-6. doi:10.1038/srep07852

Chen, X. (2004). A microRNA as a translational repressor of APETALA2 in Arabidopsis flower development. science. 303, 2022-25. doi:10.1126/science.1088060

Chen, X. (2009). Small RNAs and their roles in plant development. Annual Review of Cell and Developmental Biology. 25, 21-44. doi:10.1146/annurev.cellbio.042308.113417

Cucinotta, M., Colombo, L., I., Roig-Villanova. (2014). Ovule development, a new model for lateral organ formation. Frontiers in Pant Science. 5:117. doi: 10.3389/fpls.2014.00117

Damodharan, S., Zhao D., and Arazi T. (2016). A common miRNA160-based mechanism regulates ovary patterning, floral organ abscission and lamina outgrowth in tomato. The Plant Journal. 86, 458-71. doi:10.1111/tpj.13127.

Deb J., Bland H.M., Østergaard L. (2018) Developmental cartography: coordination via hormonal and genetic interactions during gynoecium formation. Curr Opin Plant Biol. 41, 5460. doi: 10.1016/j.pbi.2017.09.004.

Dinneny J.R., Weigel D., Yanofsky M.F. (2005). A genetic framework for fruit patterning in Arabidopsis thaliana. Development. 132, 4687-96. doi: 10.1242/dev.02062

Ding, J., Lu, Q., Ouyang, Y., Mao, H., Zhang, P., Yao, J., et al. (2012). A Long Noncoding RNA regulates photoperiod-sensitive male sterility, an essential component of hybrid rice. Proc. Natl. Acad. Sci. U.S.A. 109, 2654-59. doi:10.1073/pnas.1121374109.

Doudna, J.A., and Charpentier, E. (2014). The New Frontier of Genome Engineering with CRISPR-Cas9. Science. 346:6213. doi:10.1126/science.1258096. 
Elliott R.C., Betzner A.S., Huttner E., Oakes M.P., Tucker W.Q., Gerentes D., Perez P., Smyth D.R. (1996). AINTEGUMENTA, an APETALA2-like gene of Arabidopsis with pleiotropic roles in ovule development and floral organ growth. Plant Cell 8, 155-168.

Fatica, A., and Bozzoni, I. (2014). Long non-coding RNAs: new players in cell differentiation and development. Nature Reviews Genetics. 15, 7-21. doi:10.1038/nrg3606.

Feng, Z., Zhang B., Ding W., Liu X., Yang D.L., Wei P., et al. 2013. Efficient genome editing in plants using a CRISPR/Cas System. Cell Research. 23, 1229-32. doi:10.1038/cr.2013.114.

Ferrándiz, C., Fourquin C., Prunet N., Scutt C.P., Sundberg E., Trehin C., et al. (2010). "Carpel development" in Advances in Botanical Research, ed J.C. Kader and Michel Delseny (Burlington, MA: Academic). 1-73. doi:10.1016/B978-0-12-380868-4.00001-6

Figueiredo, D.D., Batista, R.A., Roszak, P.J., Hennig, L., Kohler, C. (2016). Auxin production in the endosperm drives seed coat development in Arabidopsis. Elife 5: pii: e20542. doi: 10.7554/eLife.20542

Franco-Zorrilla, J.M., Valli, A., Todesco, M, Mateos, I, Puga, M.I., Rubio-Somoza, I., et al. 2007. target mimicry provides a new mechanism for regulation of microRNA activity. Nature Genetics 39, 1033-37. doi:10.1038/ng2079.

Fujisawa, M., Nakano T., Shima Y., and Y. Ito. (2013). A large-scale identification of direct targets of the tomato MADS box transcription factor RIPENING INHIBITOR reveals the regulation of fruit ripening. The Plant Cell 25, 371-86. doi:10.1105/tpc.112.108118.

Gavnholt, B. and Larsen, K. (2002) Molecular biology of plant laccases in relation to lignin formation. Physiol. Plant. 116, 273 - 280.

Gubler F, Kalla R, Roberts J, Jacobsen J. (1995). Gibberellin-regulated expression of a myb gene in barley aleurone cells: evidence for Myb transactivation of a high-pl alpha-amylase gene promoter. Plant Cell 7, 1879-1891.

González-Reig S., Ripoll J.J., Vera A., Yanofsky M.F., Martínez-Laborda A. (2012). Antagonistic gene activities determine the formation of pattern elements along the mediolateral axis of the Arabidopsis fruit. PLoS Genet. 8, e1003020. doi.org/10.1371/journal.pgen.1003020.

Gorospe, M.J., Abdelmohsen, K. and Gorospe, M. (2014). Functional interactions among microRNAs and long noncoding RNAs. Semin Cell Dev Biol. 34, 9-14.

Guo, C., Xu Y., Shi M., Lai Y., Wu Xi., Wang H. et al. (2017). Repression of miR156 by miR159 regulates the timing of the juvenile-to-adult transition in Arabidopsis. The Plant Cell. 29, 1293 - 1304. doi:10.1105/tpc. 16.00975 
Hasson, A., Plessis, A., Blein, T., Adroher, B., Grigg, S., Tsiantis M., et al.. (2011). Evolution and diverse roles of the CUP-SHAPED COTYLEDON genes in Arabidopsis leaf development. The Plant Cell. 23, 54-68. doi:10.1105/tpc.110.081448.

He, J., Xu, M., Willmann, M.R., Mccormick, K., Hu, T., Yang, L., et al. (2018). Thresholddependent repression of SPL gene expression by miR156/miR157 controls vegetative phase change in Arabidopsis Thaliana. Plos Genetics. 14:e1007337. doi.org/10.1371/journal.pgen.1007337.

Heisler, M.G., Atkinson, A., Bylstra Y.H., Walsh R., and Smyth D.R.. (2001). SPATULA, a gene that controls development of carpel margin tissues in Arabidopsis, encodes a bHLH protein. Development. 128, 1089-98.

Hendelman, A., Stav R., Zemach H., and Tzahi Arazi. (2013). The tomato NAC transcription factor SINAM2 is involved in flower-boundary morphogenesis. Journal of Experimental Botany. 64, 5497-5507. doi:10.1093/jxb/ert324.

Hewezi, T., Maier, T.R., Nettleton, D., and Baum T.J. (2012). The Arabidopsis microRNA396GRF1/GRF3 regulatory module acts as a developmental regulator in the reprogramming of root cells during cyst nematode infection. Plant Physiology. 159, 321-35. doi:10.1104/pp.112.193649.

Ishida, T., Aida, M., Takada, S., and Tasaka, M. (2000). Involvement of CUP-SHAPED COTYLEDON genes in gynoecium and ovule development in Arabidopsis thaliana. Plant \& Cell Physiology. 41, 60-67. doi:10.1093/pcp/41.1.60.

Jürgens G. 2001. Apical-basal pattern formation in Arabidopsis embryogenesis. EMBO J. 20:3609-3616

Kamiuchi, Y., Yamamoto, K., Furutani, M., Tasaka, M., and Aida, M. (2014). The CUC1 and CUC2 genes promote carpel margin meristem formation during Arabidopsis gynoecium development. Frontiers in Plant Science. 5, 1-9. doi:10.3389/fpls.2014.00165.

Kapranov, P., Cheng, J., Dike, S., Nix, D.A., Duttagupta, R., Willingham A.T., et al. (2007). RNA maps reveal new and a possible classes pervasive transcription RNA function. Science. 316, 1486. doi:10.1126/science.1138341.

Karlova, R, Van Haarst, J.C., Maliepaard, C., Van De Geest, H., Bovy, A.G., Lammers, M., et al. (2013). Identification of microRNA targets in tomato fruit development using highthroughput sequencing and degradome analysis. Journal of Experimental Botany. 64, 186378. doi:10.1093/jxb/ert049. 
Karlova, R., Rosin F.M., Busscher-Lange, J., Parapunova, V., Do, P.T., Fernie, A.L., et al. (2011). Transcriptome and metabolite profiling show that APETALA2a is a major regulator of tomato fruit ripening. The Plant Cell. 23, 923-41. doi:10.1105/tpc.110.081273

Kelley ,D.R., and Gasser, S.C. (2009). Ovule development: genetic trends and evolutionary considerations. Sexual Plant Reproduction. 22, 229-234 doi: 10.1007/s00497-009-0107-2.

Kurihara Y, Watanabe Y. 2004 Arabidopsis micro-RNA biogenesis through Dicer-like 1 protein functions. Proc Natl Acad Sci USA 101:12753-12758 doi:10.1073pnas.0403115101

Laufs, P., Peaucelle, A., Morin, H., and Traas, J. (2004). MicroRNA regulation of the CUC genes is required for boundary size control in Arabidopsis meristems. Development.131, 4311-22. doi: $10.1242 /$ dev.01320

Lee, B. H., Ko, J.H., Lee, S., Lee, Y., Pak J.H., and Kim J.H. (2009). The Arabidopsis GRFINTERACTING FACTOR gene family performs an overlapping function in determining organ size as well as multiple developmental properties. Plant Physiology. 151, 655-68. doi:10.1104/pp.109.141838.

Lee, B.H., Jeon, O.J., Lee, M.M. and Kim, J.H.. (2015). Genetic interaction between GROWTHREGULATING FACTOR and CUP-SHAPED COTYLEDON in organ separation. Plant Signaling and Behavior. 10:e988071. doi:10.4161/15592324.2014.988071.

Lee, B.H., Wynn, A.N., Franks, R.G., Hwang, Y.S., Lim, J., and Kim, J.H.. (2014). The Arabidopsis thaliana GRF-Interacting Factor gene family plays an essential role in control of male and female reproductive development. Developmental Biology. 386, 12-24. doi:10.1016/j.ydbio.2013.12.009.

Lee, S.J., Lee, B.H., Jung, J.K., Park, S.K., Song, J.T., and Kim J.H.. (2017). The Growth Regulating and Growth Interacting Factor duo is essential for specification of carpel margin meristems. Plant Physiology. 176. doi:10.1104/pp.17.00960

Li, C., Zong, Y., Wang, Y., Jin, S., Zhang, D., Song, Q., et al. (2018). Expanded base editing in rice and wheat using a Cas9-adenosine deaminase fusion. Genome Biol. 19:59. https://doi.org/10.1186/s13059-018-1443-z.

Li, R., Fu, D., Zhu, B, Luo, Y., and Zhu, H. (2018). CRISPR/Cas9-mediated mutagenesis of lncRNA1459 alters tomato fruit ripening. The Plant Journal, 513-24. doi:10.1111/tpj.13872

Liang, G., He, H., Li, Y., Wang, F., and Yu, D. (2014). Molecular mechanism of microRNA396 mediating pistil development in Arabidopsis. Plant Physiology 164, 249-58. doi:10.1104/pp.113.225144. 
Liljegren S.J., Roeder A.H., Kempin S.A., Gremski K., Østergaard L., Guimil S., Reyes D.K., Yanofsky M.F. (2004). Control of fruit patterning in Arabidopsis by INDEHISCENT. Cell. 116843-53. doi.org/10.1016/S0092-8674(04)00217-X.

Liu, M., Pirrello, J., Chervin, C., Roustan, J.P., Bouzayen, M.. (2015) Ethylene control of fruit ripening: revisiting the complex network of transcriptional regulation. Plant Physiology 169:2380-2390. doi: 10.1104/pp.15.01361.

Liu, N., Tu, L., Wang, L., Hu, H., Xu, J., and Zhang, X. (2017). MicroRNA 157-targeted SPL genes regulate floral organ size and ovule production in cotton. BMC Plant Biology. 17:7. doi:10.1186/s12870-016-0969-z.

Liu, X., Huang, J., Wang, Y., Khanna, K., Xie, Z., Owen, H.A., et al. (2010). The role of floral organs in carpels, an Arabidopsis loss-of-function mutation in MicroRNA160a, in organogenesis and the mechanism regulating its expression. The Plant Journal. 62, 416-28. doi:10.1111/j.1365-313X.2010.04164.x.

Lozano, R., Giménez, E., Cara, B., Capel, J., Angosto, T. 2009. Genetic analysis of reproductive development in tomato. Int J Dev Biol. 53:1635-1648.

Lynn, K., Fernandez, A., Aida, M., Sedbrook, J., Tasaka, M., Masson, P., and Barton, M.K. (1999). The PINHEAD/ZWILLE gene acts pleiotropically in Arabidopsis development and has overlapping functions with the ARGONAUTE1 gene. Development 126: 469-481

Ma, X., Tang, Z., Qin, J., Meng, Y. (2015) The use of high-throughput sequencing methods for plant microRNA research. RNA Biol. 12, 709-19. doi: 10.1080/15476286.2015.1053686.

Mallory, A., Ducas, D., Bartel, D., and Bartel, B. (2004). MicroRNA regulation of NAC-domain targets is required for proper formation and separation of adjacent embryonic, vegetative, and floral organs. Current Biology. 14, 1035-46. doi:10.1016/j.

Mallory A.C., Bartel D.P., Bartel B. (2005). MicroRNA-directed regulation of Arabidopsis AUXIN RESPONSE FACTOR17 is essential for proper development and modulates expression of early auxin response genes. The Plant Cell. 17, 1360-75. doi.org/10.1105/tpc.105.031716.

Mallory, A.C., Hinze, A., Tucker, M.R., Bouché, N., Gasciolli, V., Elmayan, T., Lauressergues, D., Jauvion, V., Vaucheret, H., and Laux, T. (2009). Redundant and specific roles of the ARGONAUTE proteins AGO1 and ZLL in development and small RNA-directed gene silencing. PLoS Genet. 5: e1000646 doi:10.1371/journal.pgen.1000646

Manning, K., Tor, M., Poole, M., Hong, Y., Thompson A.J., King, G.J., et al. (2006). A naturally occurring epigenetic mutation in a gene encoding an SBP-Box transcription factor inhibits tomato fruit ripening. Nature Genetics. 38, 948-52. doi:10.1038/ng1841. 
Martel, C., Vrebalov, J., Tafelmeyer, P., and Giovannoni J.J.. (2011). The tomato MADS-Box transcription factor RIPENING INHIBITOR interacts with promoters involved in numerous ripening processes in a COLORLESS NONRIPENING-dependent manner. Plant Physiology. 157, 1568-79. doi:10.1104/pp.111.181107.

Mayer, A.M. and Staples, R.C. (2002) Laccase: new functions for an old enzyme.

Phytochemistry , $60,551-565$.

Möller, B., Weijers, D. (2009). Auxin control of embryo patterning. Cold Spring Harbor Perspective Biology. 1:a001545. doi:10.1101/cshperspect.a001545.

Nahar, M.A.U., Ishida, T., Smyth, D.R., Tasaka, M., and Aida, M. (2012). Interactions of CUPSHAPED COTYLEDON and SPATULA genes control carpel margin development in Arabidopsis thaliana. Plant and Cell Physiology. 53, 1134-43. doi:10.1093/pcp/pcs057

Nikovics, K., Blein, T., Peaucelle, A., Ishida, T., Morin, H., Aida, M., et al. (2006). The balance between the MIR164A and CUC2 genes controls leaf margin serration in Arabidopsis. The Plant Cell. 18, 2929-45. doi:10.1105/tpc.106.045617.

Nodine MD, Bartel DP. 2010. MicroRNAs prevent precocious gene expression and enable pattern formation during plant embryogenesis. Genes Dev. 24:2678-2692.

O'Malley, D.M., Whetten, R., Bao, W., Chen, C.-L. and Sederoff, R.R. (1993) The role of laccase in lignification. Plant J. 4, $751-757$.

Parnis, A., Cohen, O., Gutfinger, T., Hareven, D., Zamir, D., and Lifschitz, E. (1997). the dominant developmental mutants of tomato, Mouse-Ear and Curl, are associated with distinct modes of abnormal transcriptional regulation of a Knotted gene. The Plant Cell. 9, 2143-58. doi:10.1105/tpc.9.12.2143.

Park M.Y., Wu G., Gonzalez-Sulser A., Vaucheret H., Poethig R.S. (2005) Nuclear processing and export of microRNAs in Arabidopsis. Proc Natl Acad Sci USA 102, 3691-3696. doi10.1073pnas.0405570102

Peaucelle, A., Morin, H., Traas, J., and Laufs, P. (2007). Plants expressing a miR164-resistant CUC2 gene reveal the importance of post-meristematic maintenance of phyllotaxy in Arabidopsis. Development. 134, 1045-50. doi:10.1242/dev.02774.

Pratt C. (1988) Apple flower and fruit: morphology and anatomy. Hort Rev 10:273-308

Preston, J.C., and Hileman, L.C. (2013). Functional evolution in the plant SQUAMOSAPROMOTER BINDING PROTEIN-LIKE (SPL) gene family. Frontiers in Plant Science. 4, 1-13. doi:10.3389/fpls.2013.00080. 
Qin, G., Wang, Y., Cao, B., Wang, W., and Tian, S. (2012). Unraveling the regulatory network of the MADS box transcription factor RIN in fruit ripening. The Plant Journal. 70, 243-55. doi:10.1111/j.1365-313X.2011.04861.x.

Rajani S., Sundaresan V. (2001). The Arabidopsis myc/bHLH gene ALCATRAZ enables cell separation in fruit dehiscence. Curr Biol. 11, 1914-22. doi.org/10.1016/S09609822(01)00593-0.

Reinhart, B.J., Weinstein, E.G., Rhoades, M.W., Bartel, B., and Bartel, D.P.. (2002). MicroRNAs in plants. Genes and Development. 3, 1616-26. doi:10.1101/gad.1004402.of.

Reyes-Olalde J.I., Zúñiga-Mayo V.M., Serwatowska J., Chavez Montes R.A., Lozano-Sotomayor P., Herrera-Ubaldo H., Gonzalez-Aguilera K.L., Ballester P., Ripoll J.J., Ezquer I., Paolo D., Heyl A., Colombo L., Yanofsky M.F., Ferrandiz C., Marsch-Martínez N., de Folter S. (2017). The bHLH transcription factor SPATULA enables cytokinin signaling, and both activate auxin biosynthesis and transport genes at the medial domain of the gynoecium. PLoS Genet. 13, e1006726. doi: 10.1371/journal.pgen.

Ripoll, J.J., Bailey, L.J., Mai, Q.A., Wu S.L., Hon, C.T., Chapman, E.K., et al. (2015). microRNA Regulation of Fruit Growth. Nature Plants 1:15036. doi:10.1038/nplants.2015.36

Ripoll, J.J., Roeder, A.H.K., Ditta G.S., and Yanofsky M.F. (2011). A novel role for the floral homeotic gene APETALA2 during Arabidopsis fruit. Development. 138, 5167-76. doi:10.1242/dev.073031.

Rubio-Somoza, I., and Weigel D. (2011). MicroRNA networks and developmental plasticity in plants. Trends in Plant Science 16, 258-64. doi:10.1016/j.tplants.2011.03.001.

Rubio-Somoza, I., Zhou C.M., Confraria, A., Martinho, C., von Born, P., Baena-Gonzalez, E., et al. (2014). Temporal Control of Leaf Complexity by miRNA-regulated licensing of protein complexes. Current Biology. 24, 2714-19. doi:10.1016/j.cub.2014.09.058.

Salinas, M., Xing, S., Höhmann, S., Berndtgen, R., and Huijser, P. (2012). Genomic organization, phylogenetic comparison and differential expression of the SBP-Box family of transcription factors in tomato. Planta. 235, 1171-84. doi:10.1007/s00425-011-1565-y.

Scutt, C.P., Vinauger-Douard, M., Fourquin, C., Finet, C., and Dumas, C. (2006). An evolutionary perspective on the regulation of carpel development. Journal of Experimental Botany. 57, 2143-52. doi:10.1093/jxb/erj188.

Sessions, R.A., and Zambryski, P.C. (1995). Arabidopsis gynoecium structure in the wild and in ettin mutants. Development. 121, 1519-32. 
Seymour, G.B., Østergaard L., Chapman, N.H., Knapp, S., and Martin, C. (2013). Fruit development and ripening. Annual Review of Plant Biology. 64, 219-41. doi:10.1146/annurev-arplant-050312-120057.

Sieber P., Wellmer F., Gheyselinck J., Riechmann J.L., Meyerowitz E.M. (2007). Redundancy and specialization among plant microRNAs: role of the MIR164 family in developmental robustness. Development. 134, 1051-60. doi: 10.1242/dev.02817.

Silva, E.M., Silva ,G.F.F., Bidóia, B.B., Azevedo, S.M., Jesus A.F., Pino E.L., et al. (2017). MicroRNA159-targeted SIGAMYB transcription factors are required for fruit set in tomato. The Plant Journal. 92, 95-109. doi: 10.1111/tpj.13637.

Silva, G.F.F., Silva, E.M., Azevedo, A.S., Guivin, M.A.C., Ramiro, D.A., Figueiredo, C.R., et al. (2014). MicroRNA156-targeted SPL/SBP box transcription factors regulate tomato ovary and fruit development. The Plant Journal. 78, 604-18. doi:10.1111/tpj.12493.

Silva, G.F.F., Silva, E.M., Correa, J.P.O., Vicente, M.H., Jiang, N., Notini, M.M., et al. (2018) Tomato floral induction and flower development are orchestrated by the interplay between gibberellin and two unrelated microRNA-controlled modules. New Phytol. 2018 Sep 20. doi: 10.1111/nph.15492.

Skinner, D.J., Hill, A.T., Gasse, C.S. (2004). Regulation of ovule development. The Plant Cell. 16, S32-S45. doi.org/10.1105/tpc.015933

Smith, M.R., Willmann, G., Wu, T. Z., Berardini, B., Moller, D. Weijers, et al. (2009). Cyclophilin 40 is required for microRNA activity in Arabidopsis. Proc. Natl. Acad. Sci. U.S.A. 106, 542429. doi:10.1073/pnas.0812729106.

Takada, S., Hibara, K., Ishida, T., and Tasaka, M. (2001). The CUP-SHAPED COTYLEDON1 gene of Arabidopsis regulates shoot apical meristem formation. Development . 128, 1127-35.

Teotia S, Tang G. (2017). Silencing of Stress-Regulated miRNAs in Plants by Short Tandem Target Mimic (ST'TM) Approach. Methods Mol Biol. 1631:337-348.

Thompson, A.J, Tor, M., Barry, C.S., Vrebalov, J., Orfila, C., Jarvis, M.C., Giovannoni, J.J., et al. (1999). Molecular and genetic characterization of a novel pleiotropic tomato-ripening mutant. 120, 383-89.

Tsuji, H., Aya, K., Ueguchi-Tanaka, M., Shimada Y, Nakazono, M., Watanabe, R., et al. (2006). GAMYB controls different sets of genes and is differentially regulated by microRNA in aleurone cells and anthers. The Plant Journal. 47, 427-444. doi: 10.1111/j.1365313X.2006.02795.x.

Varoquaux, F., Blanvillain, R., Delseny, M., Gallois, P. (2000). Less is better: new approach for seedless fruit production. Trends in Biotechnology 18, 233-242. 
Vialette-Guiraud, A.C., Chauvet, A., Gutierrez-Mazariegos, J., Eschstruth, A., Ratet, P., Scutt, C.P.. (2016). A conserved role for the NAM/miR164 developmental module reveals a common mechanism underlying carpel margin fusion in monocarpous and syncarpous eurosids. Frontiers in Plant Science. 6:1239. doi:10.3389/fpls.2015.01239.

Vrebalov, J., Ruezinsky, D., Padmanabhan, V., White, R., Medrano, D., Drake, R., et al. (2002). A MADS-box gene necessary for fruit ripening at the tomato ripening-inhibitor (RIN) locus. Science. 296, 343-46. doi:10.1126/science.1068181.

Wang C J, Zhang S, Yu Y, Luo Y C, Ju C, Zhang Y C, Qu L H, Lucas J W, Wang X, Chen Q Y. (2014) MiR397b regulates both lignin content and seed number in Arabidopsis via modulating a laccase involved in lignin biosynthesis. Plant Biotechnology Journal 12 , pp. 1132-1142 doi: $10.1111 /$ pbi.12222

Wang, J-W. 2014. Regulation of flowering time by the miR156-mediated age pathway. Journal of Experimental Botany. 65, 4723-30. doi:10.1093/jxb/eru246.

Wang, Y., Fan, X., Lin, F, He, G., Terzaghi, W., Zhu, D.,et al. (2014). Arabidopsis noncoding RNA mediates control of photomorphogenesis by red light. Proc. Natl. Acad. Sci. U.S.A. 111, 10359-64. doi:10.1073/pnas.1409457111.

Wang, Y., Zou, W., Xiao, Y., Cheng, L., Liu, Y., Gao, S., et al. (2018). MicroRNA1917 targets CTR4 splice variants to regulate ethylene responses in tomato. Journal of Experimental Botany. 69, 1011-25. doi:10.1093/jxb/erx469.

Wang C, Jogaiah S, Zhang W Y, Abdelrahman M, Fang G J. 2018. Spatio-temporal expression of miRNA159 family members and their GAMYB target gene during the modulation of gibberellin-induced grapevine parthenocarpy Journal Experimental Botany doi:10.1093/jxb/ery172

Wang, Z., Cao, H., Sun, Y., Li, X., Chen F., Carlos A. et al. (2013). Arabidopsis paired amphipathic helix proteins SNL1 and SNL2 redundantly regulate primary seed dormancy via abscisic acid-ethylene antagonism mediated by histone deacetylation. The Plant Cell. 25,149166. doi:10.1105/tpc.112.108191.

Willemsen V and Scheres B. 2004. Mechanims of Pattern Formation in Plant Embryogenesis Annu. Rev. Genet doi: 10.1146/annurev.genet.38.072902.092231

Wu, M.F., Tian, Q., Reed, J.W. 2006. Arabidopsis microRNA167 controls patterns of ARF6 and ARF8 expression, and regulates both female and male reproduction. Development. 133:42114218. 
Wu, G., Park, M.Y., Conway, S.R., Wang, J.W., Weigel, D., and Scott, R.. 2010. The sequential action of miR156 and miR172 regulates developmental timing in Arabidopsis. Cell. 138, 750 59. doi:10.1016/j.cell.2009.06.031.

Xie, F., Jones, C.D., Wang, Q., Sun, R., and Zhang B. (2015). Small RNA sequencing identifies miRNA roles in ovule and fibre development. Plant Biotechnology Journal. 13, 355-369. doi: 10.1111/pbi.12296.

Xing, S., Salinas, M., Garcia-Molina, A., Höhmann, S., Berndtgen, R., and Peter Huijser. (2013). SPL8 and miR156-targeted SPL genes redundantly regulate Arabidopsis gynoecium differential patterning. The Plant Journal. 75, 566-77. doi:10.1111/tpj.12221.

Yamaguchi, A., Wu, M.F., Yang, L., Wu, G., Poethig, R.S., and Wagner, D. (2013). A molecular framework for auxin-mediated initiation of flower primordial. Developmental. Cell. 17, 268278. doi: 10.1016/j.devcel.2009.06.007

Yamaguchi, A., Wu, M.F., Yang, Li., Wu, G., Poethig, S.R., and Wagner, D. (2009). The microRNA-egulated SBP-box transcription factor SPL3 is a direct upstream activator of LEAFY, FRUITFULL, and APETALA1. Developmental Cell. 17, 268-78. doi:10.1016/j.devcel.2009.06.007.

Yao JL, Tomes S, Xu J, Gleave AP. (2016) How microRNA172 affects fruit growth in different species is dependent on fruit type. Plant Signal Behav. 2016;11(4):e1156833. doi: 10.1080/15592324.2016.1156833.

Zakynthinos, G., Varzakas, T., Petsios, D. (2016). Sea Buckthorn (Hippophae rhamnoides) lipids and their functionality on health aspects. Curr. Res. Nutr. Food Sci. 4, 182-94. http://dx.doi.org/10.12944/CRNFSJ.4.3.04

Zhang, G., Chen, D., Zhang, T., Duan, A., Zhang, J., He, C. (2018). Transcriptomic and functional analyses unveil the role of long non-coding RNAs in anthocyanin biosynthesis during sea buckthorn fruit ripening. DNA Res. doi: 10.1093/dnares/dsy017.

Zhang Y. C., Yu Y, Wang C Y, Li Z. Y., Liu Q et al., (2013) Overexpression of microRNA OsmiR397 improves rice yield by increasing grain size and promoting panicle branching Nature Biotechnology 31, p848-852.

Zhu, B., Yang, Y., Li, R., Fu, D., Wen, L., Luo, Y., et al. (2015). RNA sequencing and functional analysis implicate the regulatory role of long non-coding RNAs in tomato fruit ripening. Journal of Experimental Botany. 66, 4483-95. doi:10.1093/jxb/erv203

Zilberman, D., Cao, X., and Jacobsen, S.E. (2003). ARGONAUTE4 control of locus-specific siRNA accumulation and DNA and histone methylation. Science 299: 716-719 /doi.org/10.1105/tpc.110.080671 


\title{
2. MIR156-TARGETED SPL/SBPS MODULATE GIBBERELLIN (GA) RESPONSES AND REGULATE GYNOECIUM DEVELOPMENT BY CONTROLLING BOUNDARY ESTABLISHMENT AND FLORAL MERISTEM SIZE
}

\begin{abstract}
Many traits of a fully developed fruit are controlled by processes that take place early in fruit development, sometimes at floral meristem level. Locule number in tomato fruits was shown to be directly controlled by meristem size. Most knowledge regarding early stages of fruit development is based on Arabidopsis dry fruits (siliques) as a model. Conversely, little is known about the control of early stages of fleshy fruit development. We showed previously that the interaction between miR156 and gibberellin (GA) pathways controls flowering time in tomato differently from what was shown in Arabidopsis. Here we use tomato as a model to study how miR156 and GA interact in the regulation of early stages of fleshy fruit development. Both the overexpression of miR156 (156OE) and increased GA levels (or loss of DELLA function in the procera mutant) led to the production of fruits with increased locule number, associated with upregulation of the boundary gene GOBLET (GOB) in early flower buds. Plants overexpressing miRNA-resistant versions of the miR156-targeted SBP3 and SBP15 (namely rSBP3 and rSBP15) produce fruits with decreased locule number. $G O B$ is downregulated in flower buds of these plants. SBP15 attenuates GA signalling, as the expression of rSBP15 in a background with increased GA levels recovers a WT-like ovary/fruit and shoot phenotype. Interestingly, floral meristem size is increased in 156OE and procera and decreased in rSBP3 and rSBP15 plants. We found that that miR156 and GA probably regulate gynoecium development and patterning by regulating different developmental processes: boundary establishment and the establishment of the floral meristem size. Importantly, miR156-targeted SBP15 attenuates GA responses, while increasing levels of miR156 enhances them. Therefore, we propose that the miR156 module may control boundary establishment and meristem size via GA signalling.
\end{abstract}

Keywords: 1. MiR156; DELLA; Gynoecium; Fruit; SQUAMOSA

\subsection{Introduction}

Fruits are plant organs specialized in seed dispersal and present a wide morphological variety among different plant groups. Fruits can be classified according to many different morphological criteria, but one of the main dichotomies concerning fruit morphology is the distinction between fleshy and dry fruits (Giovannoni, 2004). The study of fruit development concerns the understanding of a broad number of processes that range from the transition of a vegetative to a floral meristem to the final ripening. Tomato (Solanum lycopersicum) has been used as a model to study ripening, which is the latest stage of fruit development. This is because tomato plants produce a commercially valuable fleshy fruit and the control of ripening determines many economically important traits (Seymour et al., 2013). However, most of the knowledge regarding genetic pathways that control early aspects of fruit development, such as 
gynoecium patterning, was built up based on Arabidopsis thaliana, a species that produces dry fruits (siliques). Although these early stages are important to determine final characteristics of a fully developed fruit, to date few studies have focused on understanding genetic pathways that control tomato gynoecium development. Control of meristem size is a good example of how early steps of floral development defines the final traits of fruits. Tomato mutants with altered activity of the CLAVATA-WUSCHEL circuit show increased meristem size and produce bigger fruits with extremely high number of locules (Munos et al., 2011; Xu et al., 2015a). Importantly, natural mutations in CLAVATA3 (CLV3) and WUSCHEL (WUS) were essential for tomato domestication (Muños et al., 2011; Xu et al., 2015; Zsögön et al., 2018).

We have previously shown that the microRNA156 (miR156) module controls initial steps of tomato fruit development (Silva et al., 2014). MiR156, a type of short non-coding RNA, along with its targets - members of the transcription factor family termed SQUAMOSA PROMOTER BINDING PROTEIN-LIKE (SPL or SBP-box) - defines the evolutionary conserved age-dependent floral pathway (Morea et al., 2016). Previous works have shown that the downregulation of miR156-targeted SBP/SPL in plants overexpressing miR156 (156OE) led to abnormal gynoecium and fruit development in Arabidopis and tomato (Silva et al., 2014; Xing et al., 2013). In tomato, $156 \mathrm{OE}$ gynoecia were formed by extra carpels and ectopic structures, and fruit showed undetermined growth and increased locule number (Silva et al., 2014). The overexpression of miR156 in Arabidopsis only affect gynoecium size, but no major structural alterations were reported (Xing et al., 2013). Thus, similar pathways seem to differently control the initial steps of fruit development in Arabidopsis and fleshy fruit species, such as tomato.

The phytohormone gibberellin (GA) has a complex relationship with miR156 pathway in the control of flowering time. In Arabidopsis thaliana, GA promotes flowering under noninductive conditions via degradation of DELLA proteins, a group of GRAS transcription factors that repress GA signaling (Hauvermale et al., 2012). MiR156-targeted SPL9 induces flowering by inducing MIR172b and SUPRESSOR OF OVEREXPRESSION OF CONSTANS1 (SOC1). DELLA proteins form dimers with SPL9 and interfere with its flowering inducing activity. Increased GA levels promote flowering by triggering DELLA degradation, thus releasing inhibition on SPL9 (Yu et al., 2012). However, flower development is induced by the activation of APETALA1 (AP1) by both SPL9 and DELLA proteins (Yamaguchi et al., 2014). Interestingly, increased GA levels delays flowering in tomato(G. F. F. Silva et al., 2019). Loss of function of the tomato DELLA PROCERA (PRO) increases time to flowering. The mechanistic relationship between GA and miR156 still remains to be dissected in plants that negatively 
respond to GA in terms of flowering time. Nevertheless, we recently showed that the SPL9 ortholog in tomato SBP15 does not form dimer with PRO (Silva et al., 2019).

Besides controlling flowering time, GA pathway controls some early aspects of reproductive development. DELLA was shown to control Arabidopsis inflorescence meristem size by regulating cell cycle in the rib meristem via KIP-RELATED PROTEIN2 (KRP2). DELLA-mediated control of meristem size is also conserved in barley (Serrano-Mislata et al., 2017). GA was also shown to control style length in Arabidopsis, as loss of DELLA function has led to the production of gynoecia with increased style length (Fuentes et al., 2012). Concerning initial steps of gynoecium development, GA also controls ovule number by participating in ovule primordia formation. DELLA loss of function mutants produce ovaries with less ovules, whereas expression of the dominant DELLA version rga $\Delta 17$ with a placenta-specific promoter increases the number of ovules produced in Arabidopsis (Gomez et al., 2018). DELLA also control ovule number in Brassica napus and tomato (Gomez et al., 2018).

Boundaries are established and maintained during many processes in plant development (Wang et al., 2016). They are normally characterized by a region of low cell division rate and can determine the separation between an emerging organ primordium and a meristem or two or more emerging organ primordia, for example (Wang et al., 2016). Increased number of carpel leaves initiated to form a gynoecium may result from the establishment of more boundaries in the floral meristem. Indeed, the production of gynoecia with extra carpels have been reported in tomato plants that show ectopic expression of genes related to boundary establishment and maintenance (Berger et al., 2009; Hendelman et al., 2013) A subgroup of NAC family (NAM/ $\underline{\text { ATAF/C } U C)}$ transcription factors named $N A M / C U C 3$ clade was described as boundary-defining factors in meristem and organ development. This group includes Arabidopsis CUP-SHAPED COTYLEDON1, 2 and 3 (CUC1, CUC2 and CUC3) and the tomato homolog GOBLET (GOB). (Aida, et al., 1999; Berger et al., 2009; Hasson et al., 2011; Ishidaet al., 2000; Laufs, et al., 2004; Peaucelle, et al., 2007; Rubio-Somoza et al., 2014; Sieber, et al., 2007; Takada et al., 2001; VialetteGuiraud et al., 2016). Most boundary related $N A M$ transcription factors are post-transcriptionally regulated by miR164. This miRNA was shown to restrict CUC2 and GOB expression to the boundary region in many developmental contexts (Berger et al., 2009; Laufs et al., 2004; Peaucelle et al., 2007; Vialette-Guiraud et al., 2016). Expression of boundary related $N A M$ alleles resistant to miR164 regulation leads to gynoecia developmental defects, such as extra carpels in tomato and incompletely fused carpels in Arabidopsis (Berger et al., 2009; Peaucelle et al., 2007).

Loss of $P R O$ function in pro mutants produce indeterminate fruit-like structures growing from the style end of the fruit, resembling 156OE phenotype (Carrera et al., 2012). We 
hypothesize that miR156 targets and GA may be regulating early gynoecium development through common mechanisms. Considering the lack of information on the interaction between miR156 and GA in tomato and the resemblance between 156OE and pro phenotypes, here we have analyzed the relationship between miR156 and GA pathways in the control of tomato gynoecium development.

\subsection{Material and Methods}

\subsubsection{Plant material and growth conditions}

Tomato (Solanum lycopersicum) cv. Micro-Tom (MT) was used as wild type. All transgenic and mutant plants were in MT background. 156OE (Silva et al., 2014), pro (Bassel et al., 2008) and GA20oxOE (Garcia-Hurtado et al., 2012) were described previously. Gob-4d and gob-3 alleles were introgressed in Micro-Tom from the M82 background previously described (Berger et al., 2009). Genetic transformation of rSBP3 and rSBP15 are described in a further section. Plants were grown as described by Lombardi-Crestana et al. (2012). Tissues for RT-qPCR were collected according to the stages described by Brukhin et al., (2003). For closed flower buds (CB) at stages 6 to 8 were collected. For pre-anthesis gynoecia, stage 10 flower buds were dissected and gynoecia were collected. For molecular characterization of rSBP3 and rSBP15 lines, terminal leaflet of third leaf were collected from 30-day-old plants.

\subsubsection{Crossings}

For generation of double transgenic and double mutant-transgenic plants, pollen was collected in microcentrifuge tubes and pollinated in the stigma of late pre-anthesis gynoecia. All plants were evaluated in the F1 generation except for 156OE;pro. This double transgenic-mutant was evaluated in the F2 generation, when around $1 / 4$ of the plants were homozygous for the recessive allele pro.

\subsubsection{Locule number measurements}

Fruits were cut in transverse sections to characterize number of locules. Each genotype was characterized by the percentage of the fruits that produced specific number of locules. The average of fruit number evaluated for each genotype was 200 fruits per genotype. 


\subsubsection{Generation of expression vectors and plant transformation}

Total RNA was extracted from tomato leaves with Trizol reagent (ThermoFisher Scientific), treated with Turbo DNAse (ThermoFisher Scientific) and cDNA was synthesized using ImpromII Reverse Transcriptase (Promega). Fragments for constructs were amplified from cDNA. rSBP3 was amplified and cloned in pENTR D-TOPO (ThermoFisher Scientific). UTR regions were excluded to eliminate the miR156 binding site, as this site is located in SBP3 3'UTR region. For rSBP15 we used PCR overlap to produce synonymous mutations in the miR156 binding site, which is located in the CDS. Two fragments were amplified and combined using overlapping PCR. Both PCR products were purified from the gel with QIAquick Gel Extraction Kit (Qiagen) and used as a template for overlapping PCR. This product was also cloned in pENTR D-TOPO. Cloned fragments were then recombined into pk7WG2.0 (Gateway System) using LR Clonase (ThermoFisher Scientific). Plants were transformed as described (Silva et al., 2014).

For Yeast Two Hybrid assays (Y2H), GOB (Solyc07g062840) ORF was cloned from cDNA into pENTR D-TOPO and recombined into pDEST22 and pDEST32 (ThermoFischer Scientific). PRO, SBP3 and SBP15 Y2H constructs were described by Silva et al. (2019).

\subsubsection{RT-qPCR analysis}

Total RNA was extracted from leaflets, closed flower buds and developing gynoecia, treated with DNAse and reverse-transcribed to generate first-strand cDNA, as described above. PCR reactions were performed using GoTaq qPCR Master Mix (Promega) and analyzed in a Step-OnePlus real-time PCR system (Applied Biosystems). Tomato TUBULIN (Solyc04g081490) was used as internal control. Three technical replicates were analyzed for three biological samples (each comprising 15 closed buds, 15 gynoecia or 5 leaflets), together with template-free reactions as negative controls. The threshold cycle (CT) was determined and fold-changes for each gene was calculated using the equation $2^{-\Delta \Delta c t}$ (Livak \& Schmittgen, 2001). For miRNA quantification, cDNA synthesis and qPCR reaction were performed as described (Varkonyi-Gasic et al. 2007).

\subsubsection{Yeast Two-Hybrid assays}

$\mathrm{Y} 2 \mathrm{H}$ assays were performed using the pJ69-4a strain (trp1-901; leu2-3,112; ura3-52; bis3200; gal4A; gal80A; GAL2-ADE2; lys2::GAL1-HIS3; met2::GAL7-lacZ), which bears reporter genes for HIS3 (histidine), ADE2 (adenine) and lacZ (James et al., 1996). GOB (Solyc07g062840), 
PRO (Solyc11g011260), SBP3 (Solyc10g009080) and SBP15 (Solyc10g078700) ORFs were fused to GAL4 binding domain (BD - pDEST32) and GAL4 activation domain (AD-pDEST22). Colonies were grown on SD/-Leu/-Trp/-His and SD/- Leu/-Trp/-His/-Ade plates. Five colonies were assessed for each vector combination.

\subsubsection{Confocal imaging and image analysis}

Tomato reproductive apices of approximately 10 days after germination were dissected and the first floral meristem was selected in stereomicroscope for a standardized stage. Apices bearing early floral meristems with early sepals were selected, stained by modified Pseudo-Schiff with Propidium Iodide and imaged as previously described (Serrano-Mislata et al., 2015; Bencivenga et al., 2016; Serrano-Mislata et al., 2017).

For image analysis, Python scripts and Fiji macros previously described were used to segment confocal image stacks, define the position of cells within the shoot meristem, delimit meristem cells and measure L1 volume. The function of each scripts and instructions on how to perform analysis with them are described in detail in Bencivenga et al. (2016) and Serrano-Mislata et al. (2017).

\subsubsection{Statistical analysis}

Statistical analyses were done using SigmaPlot. Data were analysed by using two-tailed Student's $\mathrm{T}$ test at $5 \%$ of significance. For RT-qPCR data, statistical tests were performed considering $\Delta \mathrm{CT}$ value of each replicate (Yung et al., 2006).

\subsection{Results}

\subsection{1. miR156 module and GA regulate fruit locule number and control GOBLET expression}

It was previously shown that miR156-targeted SPL/SBPs are critical for fruit determinacy and locule number establishment (Silva et al., 2014). Gibberellin (GA) pathway also controls fruit determinacy (Carrera et al., 2012). Tomato plants bearing PROCERA (PRO/DELLA) loss of function allele with a point mutation in the GRAS domain (procera - pro) produce fruits with fruit-like structures growing from the style end of the fruit (Figure 1A), similarly to the fruit phenotype observed for plants overexpressing the miR156 (156OE; Silva et 
al., 2014). Although these structures in the fruits have already been observed in WT plants treated with $G_{3}$, it was not shown whether altered GA signalling affects the number of locules (Carrera et al., 2012). Based on the undetermined fruit phenotypes of $156 \mathrm{OE}$ and pro mutant, our initial hypothesis was that miR156 targets and GA may act in the same pathway controlling fruit development. In order to initially address this hypothesis, we determined the number of locules in fruits produced by pro and plants overexpressing the GA20ox gene (GA20oxOE plants, which has increased levels of active GA, Garcia-Hurtado et al., 2012; Rieu et al., 2008). Fruits of both pro and GA20oxOE plants showed increased number of locules in comparison to MT (Figure 1C). Most MT fruits displayed two to three locules, whereas the majority of pro and GA20oxOE fruits showed three to four locules. The fruit phenotypes of GA-related mutant and transgenic plants was not as drastic as $156 \mathrm{OE}$, which produces most fruits with four, five and six locules (Figure 1C). Although pro produces fruit-like structures in the style end of some fruits, none of the GA20oxOE fruits exhibited this phenotype. Around 3\%, 0\%, and 17\% of pro, GA20oxOE and $156 \mathrm{OE}$ ovaries showed indeterminate structures, respectively (Figure S5D). To understand if decreased GA levels could reduce the number of locules in the fruits, we treated WT plants with the GA biosynthesis inhibitor Paclobutrazol (PBZ, Figure 1D). Strikingly, PBZ-treated plants produced fruits with decreased locule number. Most fruits produced by treated plants had two locules.

Number of locules in the fruits are a direct consequence of the number of carpels initiated by the floral meristem, considering that carpels fuse together to form gynoecia and each carpel originate a locule in the fruit (Seymour, et al., 2013). In tomato early flower bud primordia (0.8 to $1 \mathrm{~mm}$ length - Brukhin et al., 2003), carpels initially emerge separately and thus fuse to form the gynoecium. The genetic regulation of these developmental stages determines many traits of a fully developed fruit, including number of locules. To initially understand the role of the miR156 pathway in early carpel development, we assessed the expression of mature miR156 and the miR156-targeted SBP3 and SBP15 transcription factors in two stages: $1 \mathrm{~mm}$ early flower buds where carpels are emerging and fusing (here referred as closed buds - CB, Figure S1 A) and an early gynoecium stage where ovary, style and stigma are already formed but not fully elongated (here referred as pre-anthesis gynoecium - Pre, Figure S1 B). Mature miR156, SBP3 and SBP15 are significantly more expressed in CB than in Pre (Figure 1B). This expression dynamic suggests that the pathway is recruited at early gynoecia developmental stages, mostly during carpel emergence and fusion.

$G O B$ gain of function mutants (Gob-4d, for instance) were reported to produce ectopic carpels in the gynoecium, and the loss of function gob mutant (gob-3) produce fruits with fewer 
locules than WT (Berger et al., 2009). Nevertheless, it remains unclear which pathways regulate the activity of this gene in tomato fruit development, although it is already known that GOB and some of its homologs (CUC1 and CUC2) are regulated by the miR164 (Berger et al., 2009; Blein et al., 2008; Laufs et al., 2004). In fact, we have shown that overexpression of miR164 in tomato reduces locule number (Silva et al., 2014). GOB is highly expressed in CB in comparison to Pre, when carpels are already fused in the gynoecium (Figure 1B). This suggests that this gene is required for boundary establishment during early floral whorl development.
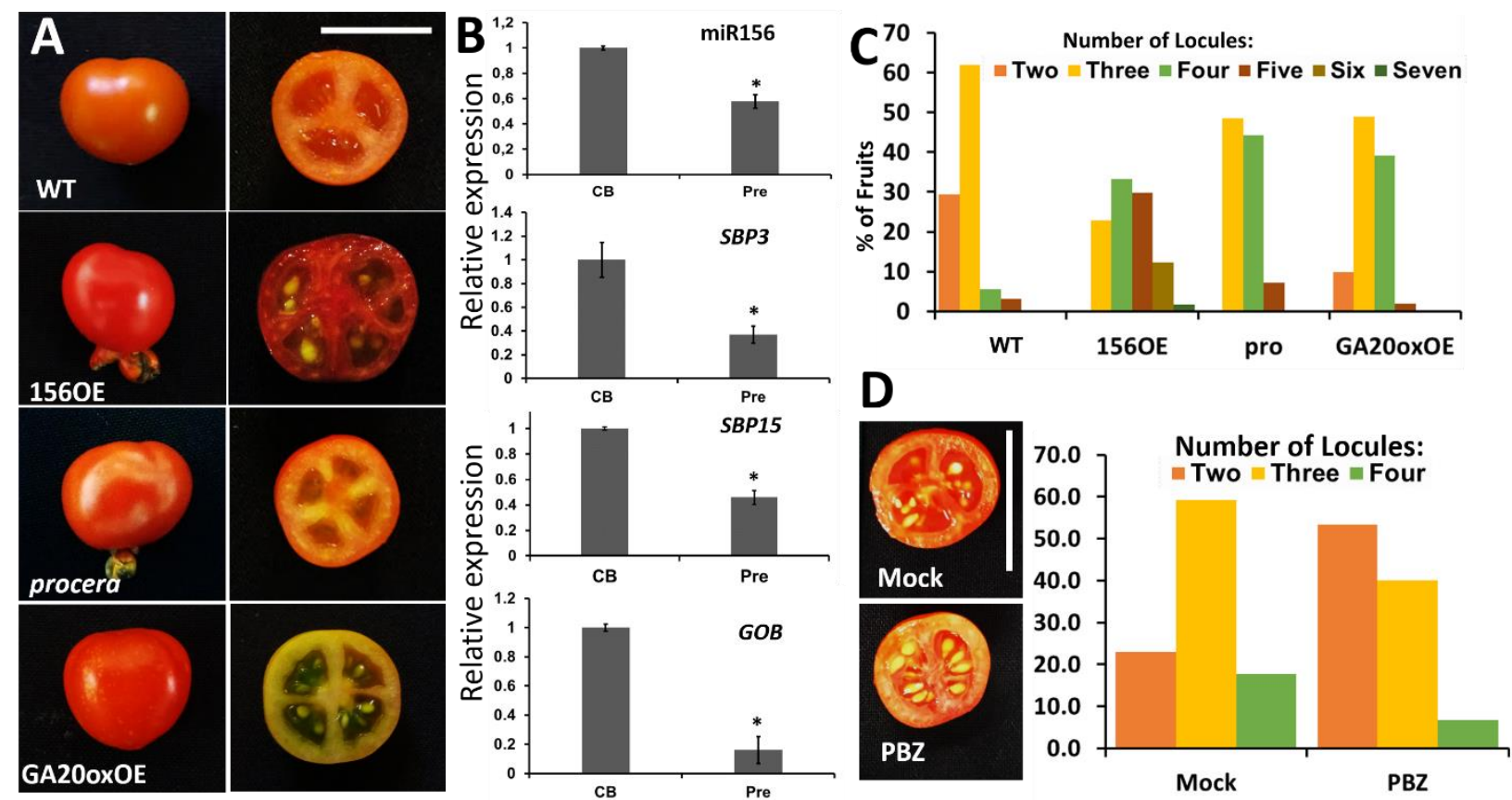

Figure 1. Phenotype of 156OE and GA-related mutant/transgenic plants and expression patterns of miR156, SBP3, SBP15 and GOB. Treatment of W'T plants with (A) Fruit morphology of 156OE and GA-related mutant/transgenic plants. (B) Relative expression of miR156 and genes by RT-qPCR. Tomato TUBULIN (Solyc04g081490) was used as an internal control for all experiments. qRT-PCR values are mean \pm s.e. of three biological replicates. Asterisks indicate a significant difference when compared with reference sample according to Student's t test (two tailed; $\left.{ }^{*} \mathrm{p}<0.05\right)$. Bar $=2 \mathrm{~cm} .(\mathrm{C})$ Percentage of fruits producing different number of locules in each genotype. ( $\mathrm{n}=150$ fruits). (D) Representative fruits of plants watered with $10^{-6} \mathrm{M}$ of Paclobutrazol and ethanol (mock) for 20 days post germination, and percentage of fruits producing different number of locules in each treatment ( $n=100$ fruits). GA, gibberellin. pro, procera. CB, closed buds; pre, pre-anthesis gynoecium. PBZ, paclobutrazol.

To better understand how increased $G O B$ activity affects gynoecium development we characterized ovary and fruit morphology of GOB gain of function heterozygous mutant (Gob-4d - allele with a point mutation in the miR164 binding site). Besides affecting gynoecium and fruit shape, ectopic expression of GOB increases number of locules in the fruit (Figure 2A and B). Most fruits produce four to six locules (Figure 2B), and the ovaries have partially fused carpels, with unfused style and stigma in the early stages of gynoecium development (Figure 2C, arrow). To shed light on the possible connection between the boundary gene GOB and the miR156 and GA pathways we assessed GOB expression in closed flower buds (CBs) of $156 \mathrm{OE}$ and pro. 
Consistent with $156 \mathrm{OE}$ and pro locule number phenotypes, GOB expression is induced in $\mathrm{CB}$ of these genotypes in comparison to MT (Figure 2D). This data suggests that SBP transcription factors and GA may regulate boundary establishment during carpel morphogenesis partially via GOB. To better clarify this idea, we are generating double mutants between miR156 module and Gob-4d, and we are treating Gob-4d mutants with paclobutrazol (PAC), a GA inhibitor (Lee et al., 1985).


Figure 2. GOB mutant phenotypes and GOB expression in $\mathrm{CBs}$ of $156 \mathrm{OE}$ and pro plants. (A) WT fruits and fruit phenotype of GOB gain of function mutant Gob-4d. Bar $=2 \mathrm{~cm}$. (B) Percentage of fruits with different number of locules in Gob-4d. ( $\mathrm{n}=150$ Fruits) (C) Ovaries of WT, Gob-4d and gob-3 (loss of function mutant - Berger et al., 2009). Bar $=2 \mathrm{~mm}$. Arrow points unfused style and stigma in a pre-anthesis gynoecium. (D) GOB transcript accumulation in 156OE and pro CBs. Tomato TUBULIN (Solyc04g081490) was used as an internal control for all experiments. qRT-PCR values are mean \pm s.e. of three biological replicates. Asterisks indicate a significant difference when compared with reference sample according to Student's t test (two tailed; *p<0.05). CBs, closed buds.

\subsubsection{Distinct miR156-targeted $S B P / S P L$ transcription factors regulate locule number and GOBLET expression}

Arabidopsis miR156-targeted SPLs and the non-targeted SPL8 have partly redundant roles in the regulation of gynoecium development (Xing et al., 2013). The overexpression of miR156 leads to a reduction in gynoecium size without major changes in organ structure, and spl8 mutant shows no obvious gynoecium phenotype. However, spl8;p35S:MIR156b plants produce 
gynoecia with altered shape, major structural changes in septum and absence of transmitting tract (Xing et al., 2013). MiR156-targeted SPL/SBP downregulation in tomato 156OE is enough to produce gynoecia with several ectopic carpels, suggesting that non-targeted SBPs may not have major roles in gynoecium development ( Silva et al., 2014). The miR156-target SPLs are subdivided into two major groups based on sequence, size and gene structure. The group that includes SPL9 and SPL15 is represented by larger proteins, due to the presence of a C-terminus domain not found in the other miR156-targeted SPLs. The transcripts of this group bear a miR156 binding site in the CDS. The group that includes SPL3 code for smaller proteins, without the C-terminus domain. These small SPLs are constituted basically by the SPL domain, and the miR156 binding site is located in the 3'UTR region of the transcripts of this group (Birkenbihl et al., 2005; Cardon et al., 1999; Xing et al., 2010). These two major groups also have divergent roles. Although both groups have specific roles in floral induction and flower development, the SPL9/15 group also control vegetative traits such as leaf morphology and leaf initiation rate (Hyun et al., 2016; Jung et al., 2012; Rubio-Somoza et al., 2014; Schwarz, et al., 2008; Usami et al., 2009; Wang et al., 2009; Wu et al., 2010; Wu \& Poethig, 2006).

The role of specific miR156-targeted SPLs in carpel development remains unknown. Analysing the functional roles of small and large miR156-targeted SPL/SBP is fundamental to understand how the roles of different clades of miR156 targets diverge in the control of carpel development. Toward this end, we generated independent lines of MT plants overexpressing miR156-resistant versions of SBP3 and SBP15 (abbreviated as rSBP3 and rSBP15, respectively). Figure 3D shows schematic representations of SBP3 and SBP15 transcripts and mutations inserted by PCR in the miR156 binding site of SBP15. rSBP3 was generated by simply not cloning the 3'UTR, where miR156 binding site is located (Figure 3D), into the construct.

Strikingly, plants from the rSBP3 and SBP15 independent lines produced fruits with reduced number of locules (Figures 3A, 3B, S2 and S3). Most fruits of these plants produce two locules, and some produce three locules (Figure 3A and 3B). Fruit shape was also changed. In all lines of both transgenics the fruits are more elongated and have a constriction in the style end (Figure 3A). Interestingly, SBP3 and SBP15 seem to have different roles in plant architecture. rSBP3 plants architecture is similar to MT, whereas rSBP15 lines produce semi-dwarf plants (Figures S2D and S3D).

For further analysis we chose the two lines of each transgenic that displayed the highest levels of transgene overexpression. To better understand whether miR156 pathway controls number of locules in the ovary/fruit we assessed $G O B$ expression in two independent lines of each transgenic plant. GOB expression was downregulated in the two lines of rSBP3 and rSBP15 
(Figure 3C). Interestingly, small and large tomato SBP transcription factors seem to have conserved roles in the transcriptional control of GOB levels in the gynoecium.
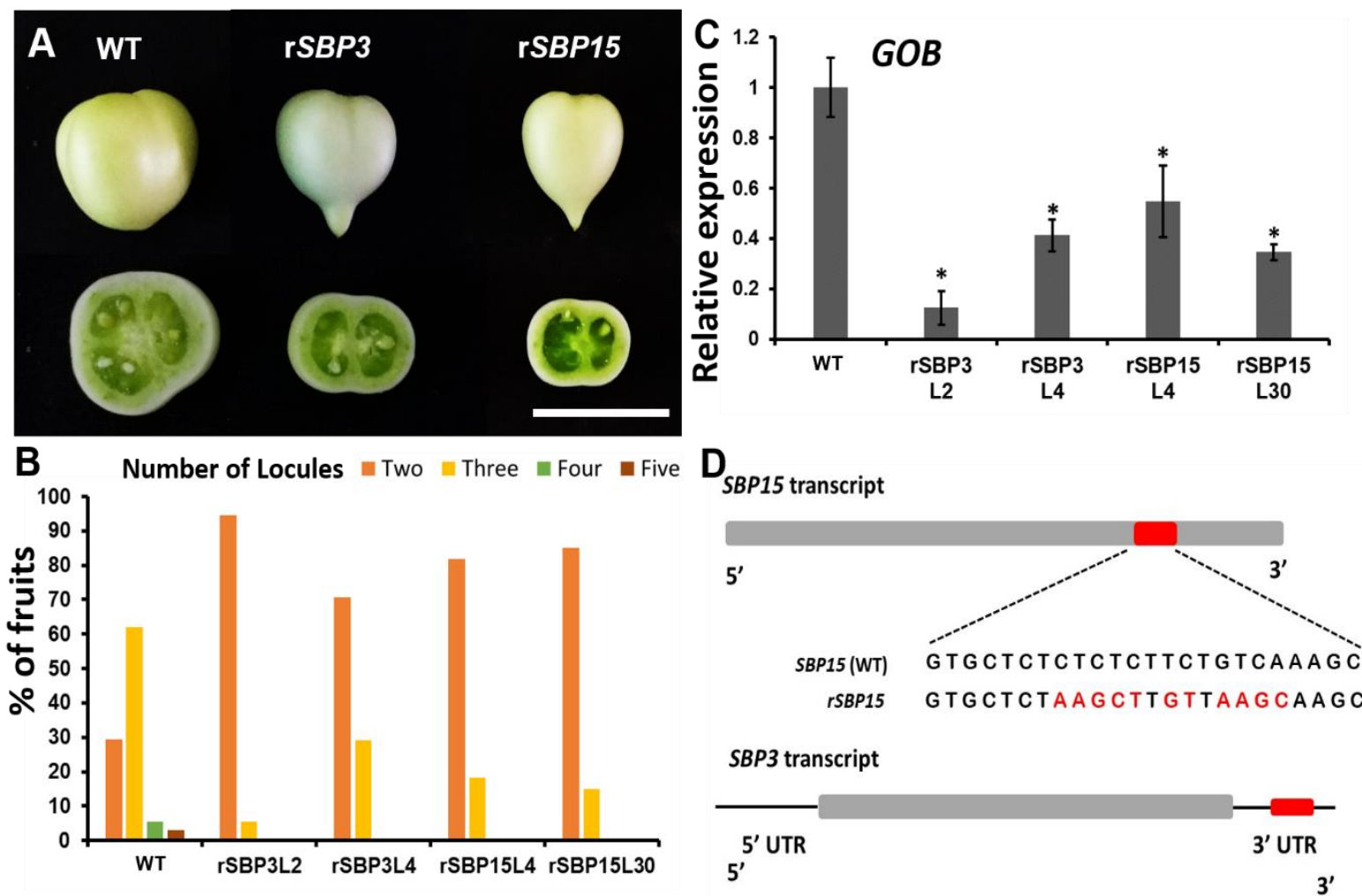

Figure 3. Overexpression of miR156-targeted SBP3 and SBP15 reduce fruit locule number and represses GOB expression in CBs. (A) WT fruits and fruit phenotype of rSBP3 and rSBP15 plants. Bar $=2 \mathrm{~cm}$. (B) Percentage of fruits producing different number of locules in two independent lines of rSBP3 and rSBP15 (rSBP3 lines \#2 and \#4; rSBP15 lines \#4 and \#30) . ( $\mathrm{n}=150$ fruits) (C) GOB transcript accumulation in two independent lines of rSBP3 and rSBP15. Tomato TUBULIN (Solyc04g081490) was used as an internal control for all experiments. qRT-PCR values are mean \pm s.e. of three biological replicates. Asterisks indicate a significant difference when compared with reference sample according to Student's t test (two tailed; ${ }^{*} \mathrm{p}<0.05$ ). (D) Schematic representation of SBP3 and SBP15 transcripts. Grey boxes represent CDS, red boxes represent miR156 binding site, black lines represent 5' and 3'UTR regions. Sequence shows mutated bases (in red) in the miR156 binding site to produce rSBP15. rSBP3 was obtained by not cloning the 3'UTR region.

\subsection{3. miR156 and GA synergistically regulate gynoecium development}

Although GA and miR156 pathways are capable of regulating GOB levels in CBs, it remains unclear whether they act in a common pathway upstream of $G O B$ or they are part of more than one pathway that converge into $G O B$ transcriptional regulation. Assessing gynoecium and fruit morphology of plants with increased GA levels or decreased DELLA activity that also has high levels of miR156 is a useful tool to initially address this question. Thus, we generated 156OE;GA20oxOE and 156OE;pro double transgenic and double transgenic mutant plants, respectively, and a synergic effect was observed. 156OE; GA20oxOE and 156OE; pro plants are taller than the respective parental plants, with modified vegetative architecture (Figure S4A and 
B) and produced extremely amorphous and undetermined fruits, with no definition of locules (Figure S4C). These fruits are composed by an amorphous pericarp mass and indeterminate fruitlike structures (Figure 4). Gynoecia are mostly formed by multiple ectopic unfused carpels and ectopic structures (Figure 4D shows the intact carpels, and, as shown in Figure S5, some carpels were dissected/removed to evidence the ectopic structures). Figure S5 shows 156OE; pro gynoecia in different developmental stages, emphasizing the ectopic structures that grow from the ovaries. Undetermined ectopic structures could be detected in gynoecia of $100 \%$ of $156 \mathrm{OE}$; pro and 156OE; GA20oxOE ovaries from the first inflorescence (Figure S5D). Considering the severity of the modifications and the frequency of ectopic structures growing from the ovary in the double mutants, it is reasonable to assume that these phenotypes exceed possible additive effects, thus characterizing the phenotype as a result of a synergistic effect.

Some studies reported synergy resulting from disruption of two steps of a pathway that converge in a node. For example, Arabidopsis auxin efflux protein PIN-FORMED1 (PIN1) mutant pin 1 form leaves and other lateral organs with slight defects. The same was observed in double mutants for auxin biosynthetic enzymes YUCCA1 and YUCCA4 (YUC1 and YUC4). Both PIN and YUC are multigene families, and other member may compensate for the aforementioned mutations. But pin1;yuc1;yuct triple mutants, which show partially disruptions in both auxin efflux and biosynthesis, fail to form normal leaves (Cheng, et al., 2007).

If control of locule number occurs via controlling boundary initiation, it is possible that miR156 and GA pathways redundantly control GOB levels. However, GOB transcript accumulation levels in 156OE; pro CBs is similar to levels observed in pro (Figure 4C), suggesting that transcriptional control of this gene may not account for the synergic effect observed. Functional and phylogenetic studies showed that Arabidopsis NAM/CUC3 group is composed by the CUP-SHAPED COTYLEDON 1,2 and 3 (CUC1, CUC2 and CUC3) and actually subdivided into 2 subgroups: NAM and CUC3. This division was based in $N A C$ domain amino acid sequences and presence/absence of miR164 binding site. CUC1 and CUC2 are grouped in the NAM subgroup whereas CUC3 is grouped in the CUC3 clade. GOB belongs to the NAM subgroup, and no tomato protein groups with CUC3. Although CUC3 is not a miR164 target CUC1, 2 and 3 have partly redundant functions the control of leaf development (Blein et al., 2008; Hasson et al., 2011). Recent data suggest NAM plus CUC3 was a single miR164-regulated lineage in the gymnosperms and diversified in NAM and CUC3 via genome duplication. Loss of miR164 binding site may have occurred later (Vialette-Guiraud et al., 2011).

A post-translational mechanism controlling heteroblasty in Arabidopsis and Cardamine birsuta was proposed to control leaf complexity by controlling dimerization of the boundary genes 
CUC2 and CUC3 (Rubio-Somoza et al., 2014). In this mechanism, titration of CUC2-CUC2 and CUC2-CUC3 dimmers control leaf complexity, and TCP4 binds to CUC2 and prevent the formation of these CUC dimmers. High levels of TCP4 during leaf organogenesis decreases the amount of CUC dimmers and leads to the production of simpler leaves (Rubio-Somoza et al., 2014). It is unknow whether such mechanism may be preserved in other organs.

To initially address this question, we tested by Yeast Two Hybrid $(\mathrm{Y} 2 \mathrm{H})$ whether PRO, SBP3 and SBP15 form dimers with GOB. Phylogenetic analysis suggest that GOB is the only CUC2 and CUC3 ortholog in tomato, thus this mechanism may operate in tomato organogenesis through the control of abundance of $G O B-G O B$ dimers. In order to initially address whether $G O B$ proteins can interact and form homodimers, we tested this interaction via $\mathrm{Y} 2 \mathrm{H}$ assays (Figure 3D). GOB is capable of auto activating the system when fused to GAL4 binding domain. However, supplementation with $10 \mathrm{mM}$ of the histidine biosynthesis inhibitor 3-amino-1,2,4triazole (3-AT) in the culture media was sufficient to prevent growth of colonies caused by auto activation (AD-, BD-GOB), whereas 3-AT did not inhibit the growth of colonies due to GOB$G O B$ interaction (AD-GOB, BD-GOB). This data suggests that $G O B$ can form homodimers. Considering this data, it is possible that proteins that bind $G O B$ are capable of disrupting $G O B-$ $G O B$ dimers by competing with other GOB proteins, as shown in Arabidopsis for CUC2 and CUC3. In order to understand whether miR156 targets or PRO could bind directly to GOB we performed more $\mathrm{Y} 2 \mathrm{H}$ assays. SBP3 and SBP15 do not interact with GOB directly. However, a strong interaction between $G O B$ and $P R O$ was detected, considering that yeasts transformed with $\mathrm{AD}-G O B$ and BD-PRO grew even with $10 \mathrm{mM} 3-\mathrm{AT}$. This result suggests that miR156 targets can control $G O B$ at transcriptional levels, while $P R O$ may control $G O B$ activity both at transcriptional and post transcriptional levels (Figures 2 and 3). Our hypothesis is that GOB-GOB homodimer promotes boundary initiation, as it happens with its Arabidopsis homologs, and PRO may be capable of binding $G O B$ and control the number of GOB-GOB dimers available in the cell. Although more experiments are still required to test this hypothesis, the phenotypes of our plants corroborate the existence of this network.

$G O B$ is post transcriptionally regulated by miR164 (Berger et al., 2009). Thus, SBP and GA pathways may regulate $G O B$ transcript levels via controlling miR164 expression. To clarify this, we assessed mature miR164 accumulation in CBs of 156OE, rSBP3, rSBP15 plants (Figure S6). Consistent with this hypothesis, miR164 levels were downregulated in 156OE CBs. However, rSBP3 and rSBP15 also showed downregulated levels of miR164. Thus, GOB downregulation in these genotypes are not explained by miR164 accumulation. This data suggests that other miR156-targeted SPL/SPBs might be involved in the regulation of GOB levels via 
miR164. We also assessed miR164 expression in 156OE; pro (Figure S6), which showed unchanged mature miR164 levels in comparison to WT. Thus, PROCERA appears to regulate $G O B$ levels independently of miR164 regulation.

Tomato recessive mutants clausa (clau) were reported to produce indeterminate fruits with fruit-like structures growing from the style end, resembling 156OE and pro phenotypes (Avivi et al., 2000) (Figure S7). This mutation was later cloned and better characterized functionally. $C L A U$ is a Solanaceae specific $M Y B$ transcription factor that attenuates cytokinin signalling and represses GOB expression in tomato leaves (Bar et al., 2015; Bar et al., 2016). $C L A U$ deregulation might be correlated with the indeterminate growth and GOB transcriptional regulation in 156OE and pro plants (Figure S7). The expression of CLAU in rSBP3 and rSBP15 CBs was not significantly different from WT. CLAU transcriptional levels may be regulated by miR156-targeted SPL/SBPs other than SBP3 and SBP15. 

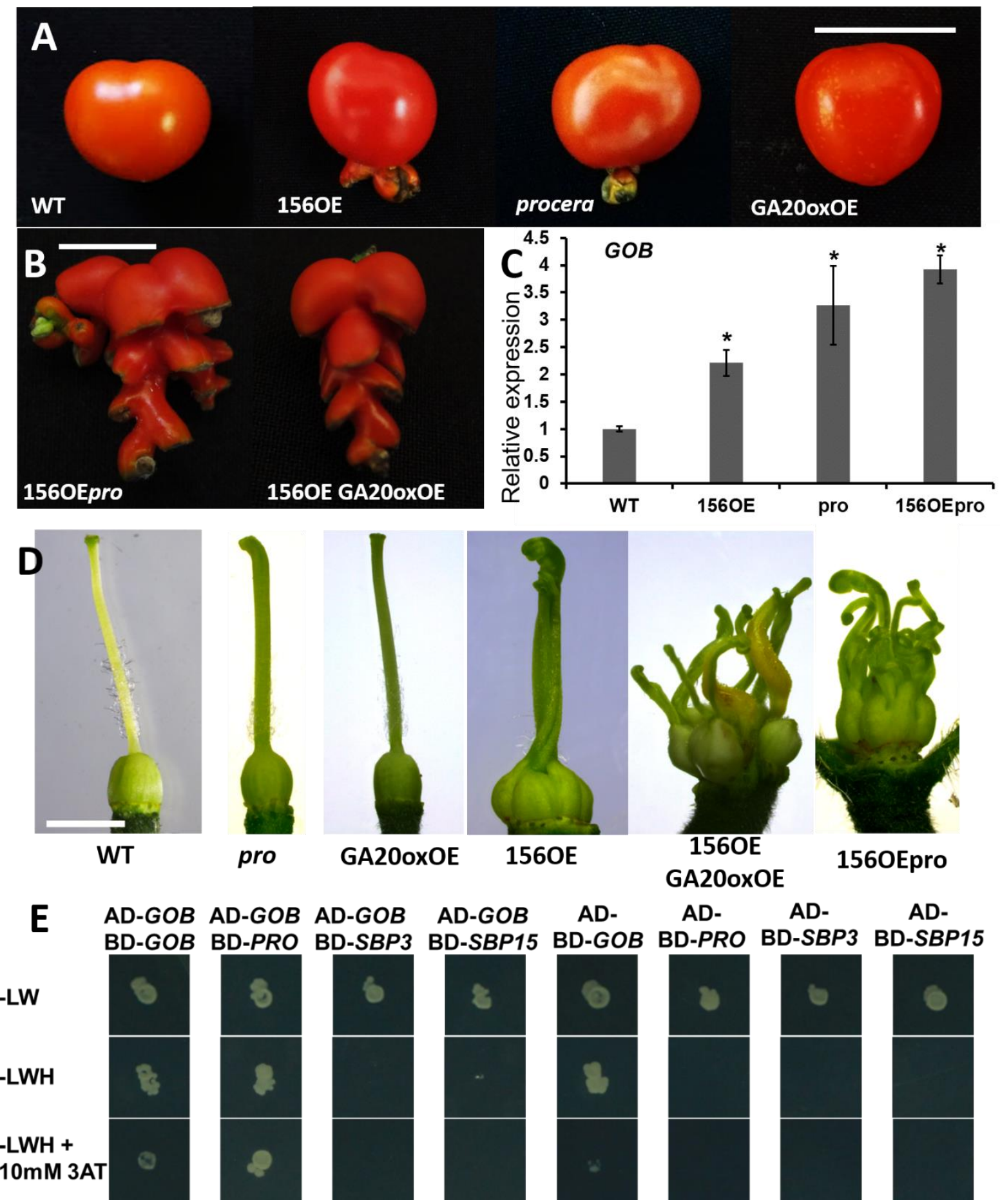

AD-GOB
BD-SBP15

AD-

AD-

$A D$ -

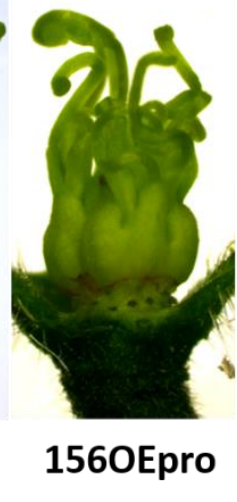

1560Epro


AD-

Figure 4. miR156 and GA pathways synergistically regulate gynoecium and fruit development. (A) and (B) Fruit morphologies of 156OE, pro and GA20oxOE plants compared to 156OE;pro and 156OE;Ga20oxOE. Bars $=2 \mathrm{~cm}$. (C) GOB expression in 1560E;pro in comparison to GOB levels in WT, 156OE and pro.Tomato TUBULIN (Solyc04g081490) was used as an internal control for all experiments. qRT-PCR values are mean \pm s.e. of three biological replicates. Asterisks indicate a significant difference when compared with reference sample according to Student's t test (two tailed; ${ }^{*} \mathrm{p}<0.05$ ). (D) Gynoecium phenotypes of mutants, transgenic and double mutanttransgenic plants. Bar $=2 \mathrm{~mm}$. (E) Two-hybrid system $(\mathrm{Y} 2 \mathrm{H})$ assays. GOB was fused to both GAL4 binding domain (BD) and GAL4 activation domain (AD). SBP15, SBP3 and PRO were fused toGAL4 BD. Colonies were examined on $\mathrm{SD} / \mathrm{-Leu} /$-Trp/-His and $\mathrm{SD} /$-Leu/-Trp/-His/-Ade plates. Five clones were analysed for each interaction, and one is shown. 


\subsubsection{SBP15 attenuates $G A$ responses}

Increased plant height is a phenotype related to increased GA signalling in many plant species (Daviere \& Achard, 2013). DELLA was shown to control plant height by blocking the activity of Class I TCP transcription factors in Arabidopsis shoot apex (Davière et al., 2014). The extreme plant height and fruit phenotypes observed in 156OE;pro and 156OE;GA20oxOE (Figures 4 and S6) are consistent with the hypothesis that miR156-targeted SPL/SBPs may modulate GA responses. If SPL/SBP downregulation in $156 \mathrm{OE}$ is capable of increasing GA responses, upregulation of specific SPLs may be capable of attenuating GA response or decrease GA sensitivity. To test this hypothesis, we expressed rSBP3 and rSBP15 in backgrounds with plants showing increased GA biosynthesis. We generated rSBP3;GA20oxOE and rSBP15;GA20oxOE double transgenic plants. Strikingly, high SBP15 levels attenuate GA responses. Instead of typically tall plants, rSBP15; GA20oxOE are also semi-dwarf like rSBP15 plants (Figure 5A). A WT-like phenotype in terms of locule number distribution was rescued in the fruits (Figure 5C). This phenotype rescue was not observed in rSBP3;GA20oxOE plants. These plants display phenotypes resembling GA20oxOE plants both in terms of plant height and fruit locule number (Figure 5). This data points to the divergent regulatory roles of large and small SPL/SBPs in the regulation of gynoecium development. Increased levels of both transcription factors are capable of downregulating $G O B$ and decrease number of carpels initiated in the floral meristem. However only SBP15 is capable of attenuating GA responses. It is possible that SBP15 regulates many aspects of plant development via modulation of GA responses.

Our data indicates that a point of interaction between miR156 and GA pathways is the control of GA responses by SBP15. To understand if GA pathway have any type of role in the transcriptional control of miR156 targets, we assessed the expression of four miR156 targets in pro CBs: SBP2, SBP3, SBP10 and SBP15 (Figure S8). Interestingly, SBP15 is the only gene that was considerably upregulated in pro (Figure S8). Apparently, there is a feedback loop in which SBP15 negatively regulates GA responses while PRO represses SBP15. It may be a mechanism related to the fine-tuning of GA responses in plant cells. To better test this hypothesis, we are currently treating WT, 156OE, and rSBP15 plants with paclobutrazol (PAC), an inhibitor of GA biosynthesis (Jung et al., 2012). 

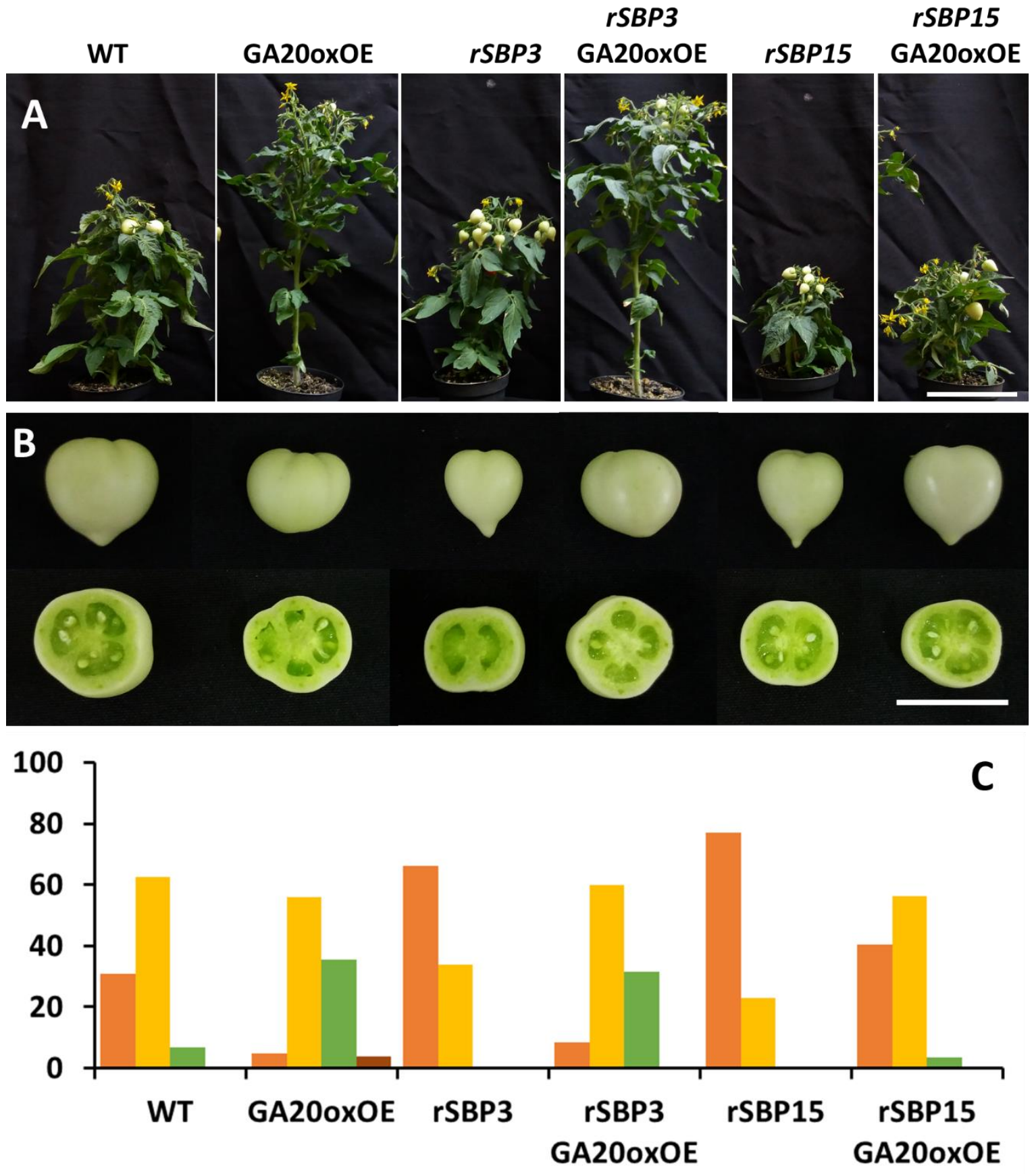

Figure 5. High SBP15 levels attenuate GA responses in tomato. Representative shoot (A) and fruits (B) of WT, GA20oxOE, rSBP3, rSBP3;GA20oxOE, rSBP15, and rSBP15;GA20oxOE. GA-induced shoot elongation is completely inhibited in rSBP15;GA20oxOE. Bars $=7 \mathrm{~cm}$. (C) Percentage of fruits producing different number of locules in WT, GA20oxOE, rSBP3, rSBP3;GA20oxOE, rSBP15, and rSBP15;GA20oxOE. ( $\mathrm{n}=100$ fruits).

\subsubsection{Among the miR156-targeted SPL/SBPS, SBP15 has a major role in controlling gynoecium development}

Higher levels of SBP3 and SBP15 downregulate GOB transcript levels in $\mathrm{CBs}$, although they diverge functionally concerning their role in modulating GA responses. Thus, these genes seem to have partly overlapping functions in regulating gynoecium development, but they are not 
completely redundant. To further investigate specific roles of each of these transcription factors among other miR156-targeted SPL/SBPs we generated 156OE;rSBP3 and 156OE;rSBP15 double transgenic plants (Figure 6 and S9). These plants present downregulated levels of all miR156-targeted SPL/SBPs (Silva et al., 2014) except for one specific (either SBP3 or SBP15). While 156OE;rSBP15 rescued WT-like gynoecium morphology and fruit locule number,156OE;rSBP3 showed only a partial phenotype recovery. Interestingly, 156OE;rSBP3 produced fruits with less locules than $156 \mathrm{OE}$ but did not recover a WT-like frequency. Furthermore, ovary shape was not totally recovered. Part of the rSBP3;156OE gynoecium show incomplete fused style and stigma (Figure 6, arrow). SBP15 is probably a major miR156 target controlling gynoecium development and it interacts with more pathways in this regulation. Moreover, plant shoot architecture was more modified by the expression of rSBP15 in a background overexpressing miR156. Instead of the 156OE-like bushy and highly branched vegetative architecture (Silva et al., 2014), 156OE;rSBP15 plants show a less branched shoot architecture (Figure S9). It has been described in Arabidopsis that the SBP15 orthologs SPL9 and SPL15 controls shoot branching (Schwarz et al., 2008). On the other hand, 156OE;rSBP3 plants did not show altered plant architecture in comparison with 156OE plants (Figure S9). We have recently obtained rSBP15;Gob-4d double mutant transgenic plants and we are currently assessing fruit and ovary phenotype to understand whether GOB downregulation by high SBP15 levels can attenuate Gob-4d phenotype. 


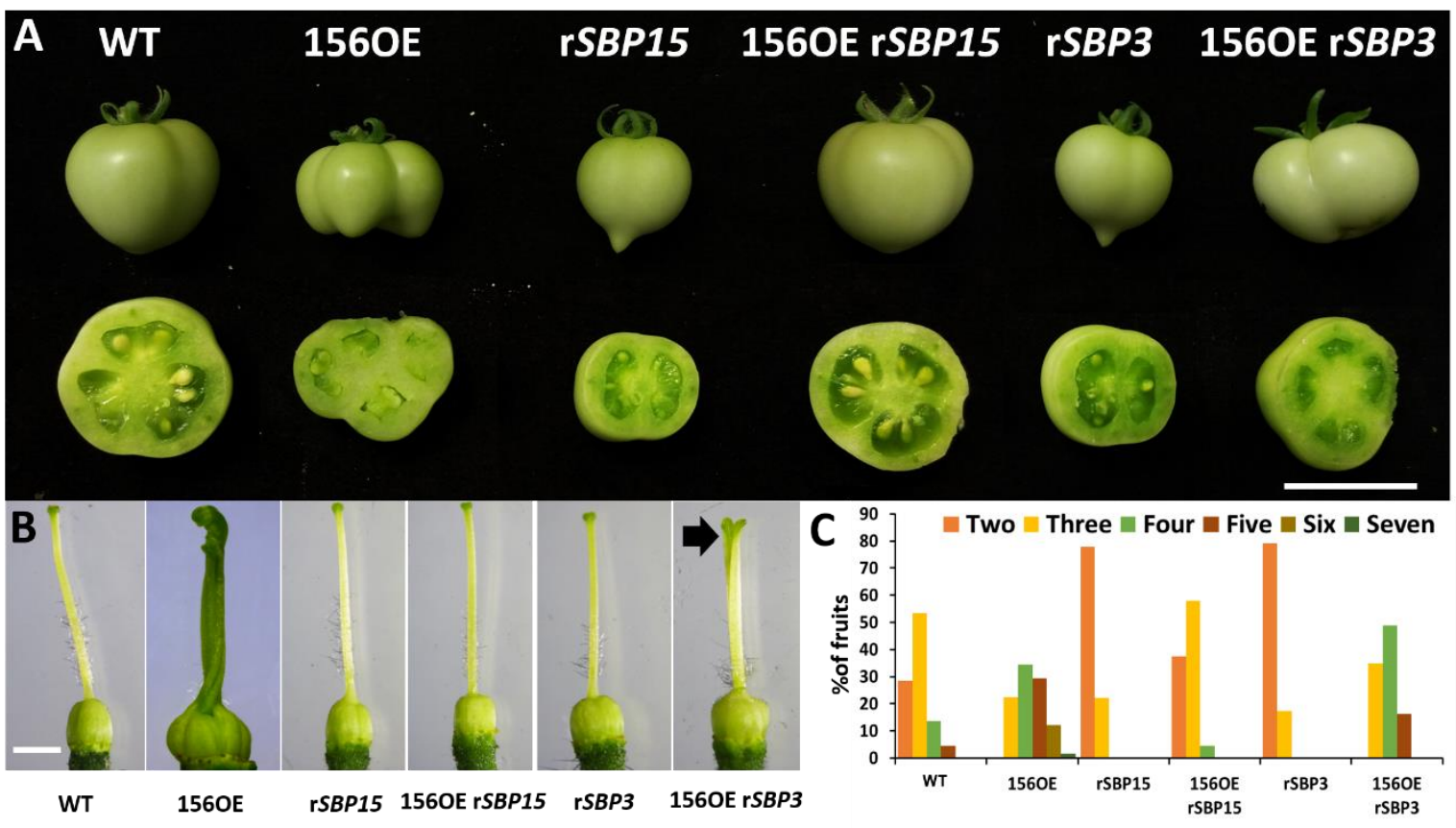

Figure 6. Expression of a miR156-resistant version of SBP15 recovers WT-like fruit phenotype in 156OE plants. (A) and (B) Representative WT, 156OE, rSBP15, 156OE;rSBP15, rSBP3 and 156OE;rSBP3 fruits and ovaries. Arrow in (B) indicates unfused region in the style and stigma. (C) Percentage of fruits producing different number of locules in each genotype. ( $\mathrm{n}=80$ fruits). (A) Bar $=2 \mathrm{~cm}$. (B) Bar $=2 \mathrm{~mm}$.

\subsection{6. miR156 and GA regulate floral meristem size}

We showed that regulation of boundary establishment and maintenance is an important factor in the control of locule number in tomato ovary/fruit. Furthermore, regulation of meristem size was also associated with the production of flowers with increased number of whorls (Muños et al., 2011; Xu et al., 2015). As a consequence of increased number of carpels, tomato plants produce fruits with increased number of locules in genotypes where disruption of CLAVATA-WUSCHEL circuit lead to increased meristem size (Muños et al., 2011; RodriguezLeal et al., 2019; Xu et al., 2015a). To understand if higher locule number observed in genotypes associated with miR156 and GA pathways is a result of the control of meristem size, we measured floral meristem dome of pro, 156OE, rSBP3 and rSBP15 plants We also measured Gob$4 d$ floral meristem size to understand if manipulation $G O B$ activity may affect meristem size and shed light over the relationship between control of boundary establishment and control of meristem size. In order to precisely measure floral meristem size, we first established in tomato the modified Pseudo-Schiff Propidium Iodide and image analysis techniques described for Arabidopsis (Bencivenga et al., 2016; Serrano-Mislata et al., 2017) (Figure 7). After successfully establishing this approach for tomato, we used the volume of L1 layer of meristem dome as a proxy of meristem surface area. Cells considered for measurement are highlighted in red in Figure 7A. Representative raw confocal images of each genotype are shown in Figure S10. Both 
pro and 156OE showed increased meristem surface area, while rSBP3 and rSBP15 showed decreased meristem area. Thus, variation in fruit locule number seems to be correlated with floral meristem size in these genotypes. Interestingly, Gob $4 d$ L1 volume did not vary significantly in comparison to WT. This suggests that changes in boundary formation do not affect meristem size. MiR156 and GA pathways seem to control gynoecium development by regulating different stages of floral development. In early floral meristem development, these pathways may regulate genes associated with meristem maintenance may be regulated. In further stages, when boundary establishment determine the number of carpels initiated, GOB seems to be controlled by GA and miR156.

It is worth to note that meristem increasing in $156 \mathrm{OE}$ and pro and decreasing in rSBP3 and rSBP15 is also associated with variation in cell size. Moreover, 156OE and pro has significantly more cells in the meristems. Thus, the area of the meristems is larger both due to increased cell size and increased number of cells in the meristem. However, rSBP3 and rSBP15 meristems show smaller surface cells but does not have a significantly lower number of cells in the meristem. 


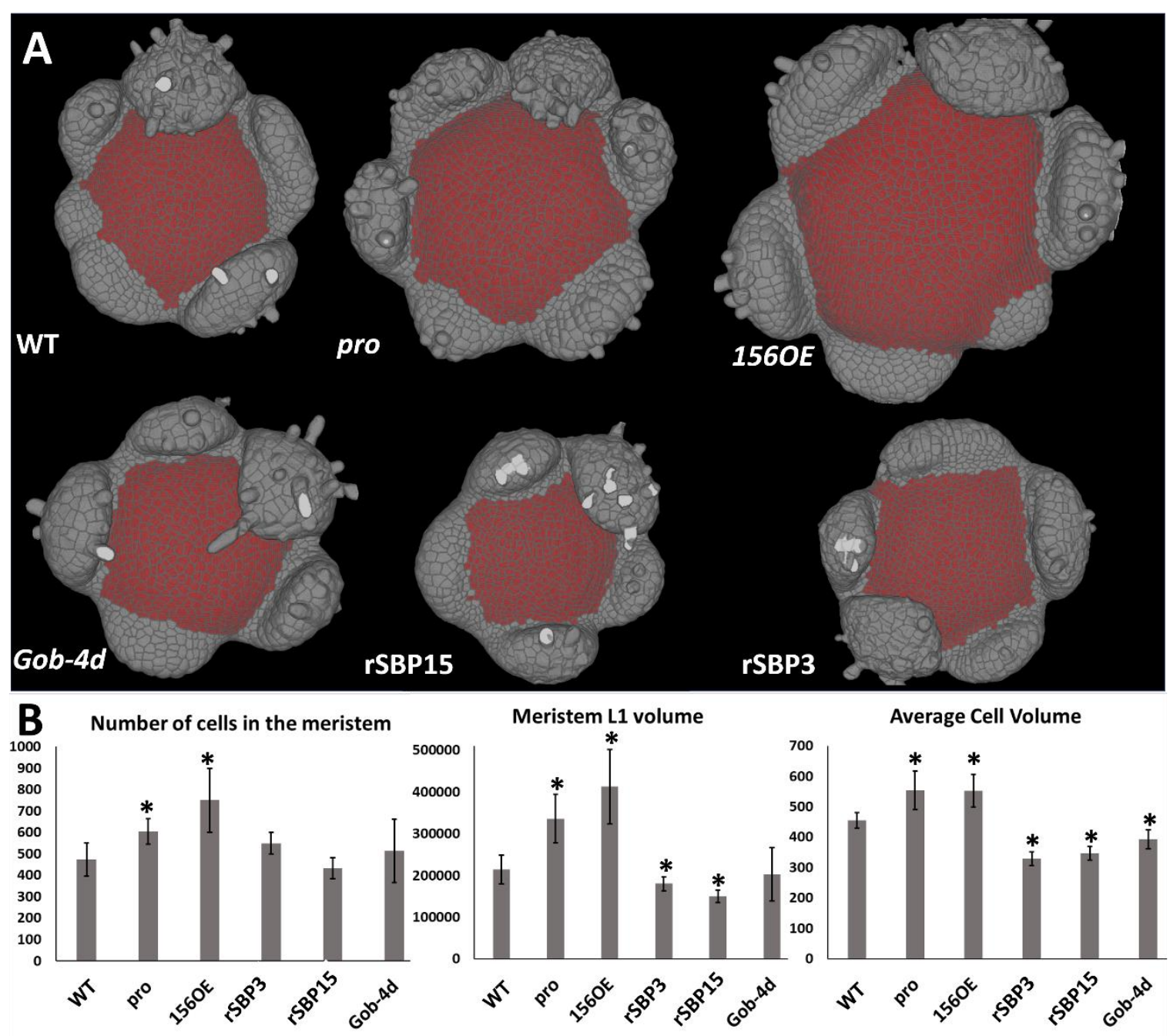

Figure 7. miR156 and GA pathways control floral meristem size in tomato. (A) 3D reconstruction of processed confocal stacks of floral meristems stained with mPS-PI. Representative floral meristems of W'T, pro, 156OE, Gob4d, rSBP15 and rSBP3 plants. Cells highlighted in red were considered for measurement. (B) Number of cells in the meristem, Meristem L1 volume and average cell volume in each genotype. Asterisks denote significant difference from control (WT) according to Student's T test at 5\% significance.

\subsection{Discussion}

Some aspects of the complex interaction between miR156 and GA has been relatively well characterized in the control of Arabidopsis flowering time. However, GA response itself is very complex and pleiotropic, and molecular mechanisms of DELLA action are still poorly understood. Furthermore, we know from our previous study that miR156 and GA interaction differ between tomato and Arabidopsis in a key step of flowering time control: in Arabidopsis GA promotes flowering by releasing DELLA-bound SPL9 proteins, whereas in tomato GA delays flowering time and DELLA does not interact with SBP15.

The overexpression of miR156 has major effects on gynoecium and fruit development, probably related to mis-regulation of specific sets of genes (Silva et al., 2014). Here we show that miR156 and GA probably regulate gynoecium development and pattern by regulating at least two 
temporally separated developmental processes: boundary establishment and meristem size. Importantly, miR156-targeted SBP15 attenuates GA responses. Thus, miR156 module may regulate many developmental processes by modulating GA responses, including the control of cell expansion.

Boundaries shape many aspects of plant body. NAM/CUC genes are expressed in boundary zones and the loss of their function leads to the production of adjacent fused organs or less complex organs. Also, their ectopic expression produces defects related to increased boundary establishment, such as more complex leaves and gynoecia with extra carpels (Berger et al., 2009; Hendelman et al., 2013). They were proposed to be key players in boundary establishment, although their downstream targets and related mechanisms are still poorly understood. $N A M / C U C$ mutants produce gynoecium developmental defects in several species. In the Medicago trunculata MtNAM null mutants, gynoecia is composed by unfused carpels with exposed ovules (Cheng et al., 2012). Arabidopsis cuc1;cuc2 double mutants show unfused septum and fewer ovules (Ishida et al., 2000). Unlike GOB, another miR164-targeted tomato boundaryrelated NAM gene SINAM2 is expressed only after carpels had already been fused, and most likely acts in boundary maintenance rather than boundary establishment. Overexpression of miR164-resistant SINAM2 leads to the production of extra carpels, similarly to Gob-4d phenotype (Hendelman et al., 2013). Two important questions regarding boundary establishment is when and how CUC-like genes are active in some developmental processes? In tomato, SBP3 and SBP15 seem to be responsible for controlling GOB activity during carpel development. MiR156, SBP3, SBP15 and GOB are recruited early in $\mathrm{CB}$ and less expressed in Pre-anthesis gynoecia (Figure 1C). This coexpression pattern, together with ovary/fruit phenotypes of 156OE, rSBP3, rSBP15 and Gob-4d corroborates with the hypothesis that recruitment of miR156 module in early stages of carpel development is necessary to fine tune $G O B$ levels or limiting its physical expression domains to specific regions during flower development. GOB downregulation in rSBP3 and rSBP15 may account for less boundary zones established during carpel development, thus leading to the emergence of less carpels from the flower meristem and production of fruits with decreased locule number. Transcriptional regulation of $N A M / C U C$ genes by SPL/SBP genes have not been reported so far. The only relationship between miR156 targets and NAM/CUC was reported in a different developmental context. Arabidopsis SPL9 was shown to repress LATERAL SUPRESSOR (LAS) expression in boundary establishment during axillary bud formation, whereas CUC2 directly induces LAS expression (Tian et al., 2014).

Rubio-Somoza et al. (2014) proposed a mechanism by which CUC2 and CUC3 control leaf complexity, and their activity is indirectly controlled by SQUAMOSA PROMOTER- 
BINDING PROTEIN-LIKE 9 (SPL9), which is a SBP15 ortholog. This mechanism promotes temporal control of leaf complexity. CUC2 and CUC3 dimmers are effectors of boundary establishment, and control of CUC2-CUC3 and CUC2-CUC2 dimer abundance modulates boundary initiation in leaf margins and thus leaf complexity. CUC2-CUC2 dimers are titrated by TCP4. TCP4 interacts with CUC2, preventing the formation of CUC homo and heterodimers. The miR156 pathway adds another level of regulation in this mechanism: as miR156 expression decreases through plant age, its targets accumulate more in the tissues. miR156-targeted SPL9 is capable of binding to TCP4 and disrupt TCP4-CUC2 heterodimers, thus releasing CUC2 to form CUC2-CUC2 and CUC2-CUC3 dimers, which promote leaf complexity. So, when more SBP9 is available in the cells to bind to TCP4, more CUC dimers are available to promote complexity. However, it is unknown whether such mechanism is conserved in different developmental contexts or different species. It is unlikely that this mechanism involving the homologs of TCP4 (LANCEOLATE - LA), CUC2 (GOB) and SBP9 (SBP15) is conserved in tomato. We showed in a previous study that $L A$ proteins do not interact with $S B P 15$ by Yeast Two Hybrid $(\mathrm{Y} 2 \mathrm{H})$ and Bimolecular fluorescence complementation (BiFC) assays (Silva et al., 2019). However, a similar post translational mechanism may operate in boundary establishment also in tomato

Besides regulating $G O B$ transcriptional levels, $P R O$ proteins interact with $G O B$. If $G O B-$ $G O B$ homodimers are necessary to transactivate downstream targets, as described for CUC2CUC2 and CUC2-CUC3 dimers (Rubio-Somoza et al., 2014), it is possible that PRO controls boundary establishment by hijacking GOBLET proteins and preventing GOBLET homodimer formation. It is also possible that PRO interacts with GOB DNA binding domain and blocks its function, as it happens between Arabidopsis DELLAs and PHYTOCHROME INTERACTING FACTOR3 and 4 (De Lucas et al., 2008; Feng et al., 2008). Both situations explain how boundary formation in 156OE; pro and 156OE;GA20oxOE may be increased by transcriptional upregulation of $G O B$ and also increased amount of available $G O B-G O B$ dimers. The upregulation of $G O B$ in pro may also be related to the increased amount of GOB-GOB, considering that in CUC2-CUC2 homodimers are capable of inducing CUC3 expression in Arabidopsis leaf development (Rubio-Somoza et al., 2014). The point mutation in VHIID domain in pro may have reduced $P R O$ ability to interact with other proteins, thus releasing more $G O B$ proteins to form homodimers and establish more boundaries during carpel emergence. Gob-4d displays diffuse expression of $G O B$ in multiple points of leaf primordia instead of determining sharp boundaries of GOB expression with adjacent GOB-less region (Berger et al., 2009). Therefore, instead of producing leaves with more leaflets, these leaflets fail to separate and form leaf lobes. This may be the case of carpel leaves in 156OE; pro and 156OE ;GA20oxOE plants, in which aberrant 
fruits do not have defined locules (Figure 4 and S4). The induction of GOB activity by downregulating several SPL/SBPs and mutated PRO in the same plant may lead to initiation of multiple partially fused aberrant carpels that originate the fruit-like organs in these genotypes.

Two major loci involved in tomato domestication are related to increased fruit size and number of locules: FASCLATED (FAS) and LOCULE NUMBER (LC). Both lc and fas mutants produce fruits with increased number of locules, and they synergistically contribute to fruit phenotypes extremely high locule number (Muños et al., 2011; Rodríguez et al., 2011). These genes were later cloned, and fas was shown to bear a mutation negatively affecting CLAVATA3 expression, while lc was due to two Single Nucleotide Polymorphisms (SNPs) downstream WUSCHEL (WUS) that induced WUS expression (Munos et al., 2011; Xu et al., 2015a). Both mutations lead to increased meristem size. The changes in meristem size reported in pro, $156 \mathrm{OE}$, rSBP3 and rSBP15 are probably associated with the number of locules produced by fruits, as genotypes with larger meristems produced more locules in the fruits and genotypes with smaller meristem produced simpler fruits, with reduce locule number. The downstream targets of SPL3 and 15 that may control meristem size remains unknown. Interestingly, it was recently shown that miR156-targeted SPL transcription factors control shoot apical meristem (SAM) size by promoting WUS expression independent of CLV1-CLV3 signalling (Fouracre \& Poethig, 2019). Loss of miR156/157 function lead to the production of increased SAM, and loss of SPL2/9/10/13 and SPL15 in sextuple mutants induced increases in SAM size. We are currently analysing whether this mechanism is conserved in the establishment of tomato floral meristem size.

The relationship between miR156 targets and GA signalling is complex and so far, most available information was unveiled in Arabidopsis, mainly concerning the control of flowering time. Data from our previous study is consistent with the hypothesis that miR156 pathway modulates GA responses in the control of flowering time, although it was not demonstrated functionally (Silva et al., 2019). Here we provide functional evidence that miR156-targeted SBP15 blocks GA effects in tomato plants, with evidences on plant architecture and fruit morphology. It was recently shown that the upregulation of rice OsSPL14 (SBP15 ortholog) also blocks GA effects in seed germination, seedling growth and disease susceptibility (Liu et al., 2019).

DELLA proteins were already reported to control inflorescence meristem size in Arabidopsis by targeting the cell cycle regulator KRP2, without interfering with the WUSCHELCLAVATA pathway (Serrano-Mislata et al., 2017). Like in tomato, DELLA loss of function plants exhibited increased meristem size. The downstream targets of PRO and SBP15 remain uncharacterized in tomato meristem maintenance. If SBP15 controls WUS (as in rice) and PRO 
controls meristem size without affecting CLV-WUS, it is reasonable to predict that miR156targeted SBP15 and PRO synergistically control meristem size. Furthermore, SBP15 controls the response to GA. Thus, the overexpression of miR156 in a background with loss of PRO function or increased GA levels may lead to even more increased meristem size due to increased GA responses, adding another layer of complexity in the relationship between miR156 and GA pathways. Synergic effect may also occur in double mutants when the effect of one of the mutations confer more sensitivity to the effects of the other (Pérez-Pérez, Candela, \& Micol, 2009).

Cell size had a significant contribution in meristem size variations seen in 156OE, pro, rSBP3 and rSBP15 plants Molecular regulation of cell size homeostasis in plant meristems remains largely unknown. It was proposed that cell size in Arabidopsis SAM is regulated by a balance between cell growth and cell division. Also, the homeostatic size of the cells in meristematic tissues depends on cell growth rate (Jones et al., 2017). MiR156 module controls plastochron length, and double mutants spl9 spl15 were shown to have shorter plastochrons (Wang et al., 2008). The reasons why miR156 and GA control cell size in the floral meristem remain an opened question. However, studying the relationship between organ initiation rate, cell growth and cell division may provide interesting insights.

This is the first study to date that explores the complex relationship between miR156 pathway and GA in the control of gynoecium development. It will be interesting to understand how SBP15 controls GA responses at cell and molecular levels. It will shed light on much of the fruit phenotype reported in $156 \mathrm{OE}$ is due to increased sensitivity to GA and how much is related to the downregulation of other SBPs. 


\subsection{Supplemental material}


Figure S1. Developmental stages collected for RT-qPCR analysis. (A) Early flower buds at stages 6 to 8, according to Brukhin et al. (2003), here referred as CB. Bar $=1 \mathrm{~mm}$. (B) Developing gynoecium from dissected stage 10 floral bud. Bar $=2 \mathrm{~mm}$. (C) Fully developed ovary dissected from an yellow opened flower at anthesis. This final developmental stage was not collected for RT-qPCR analyses. Bar $=2 \mathrm{~mm}$
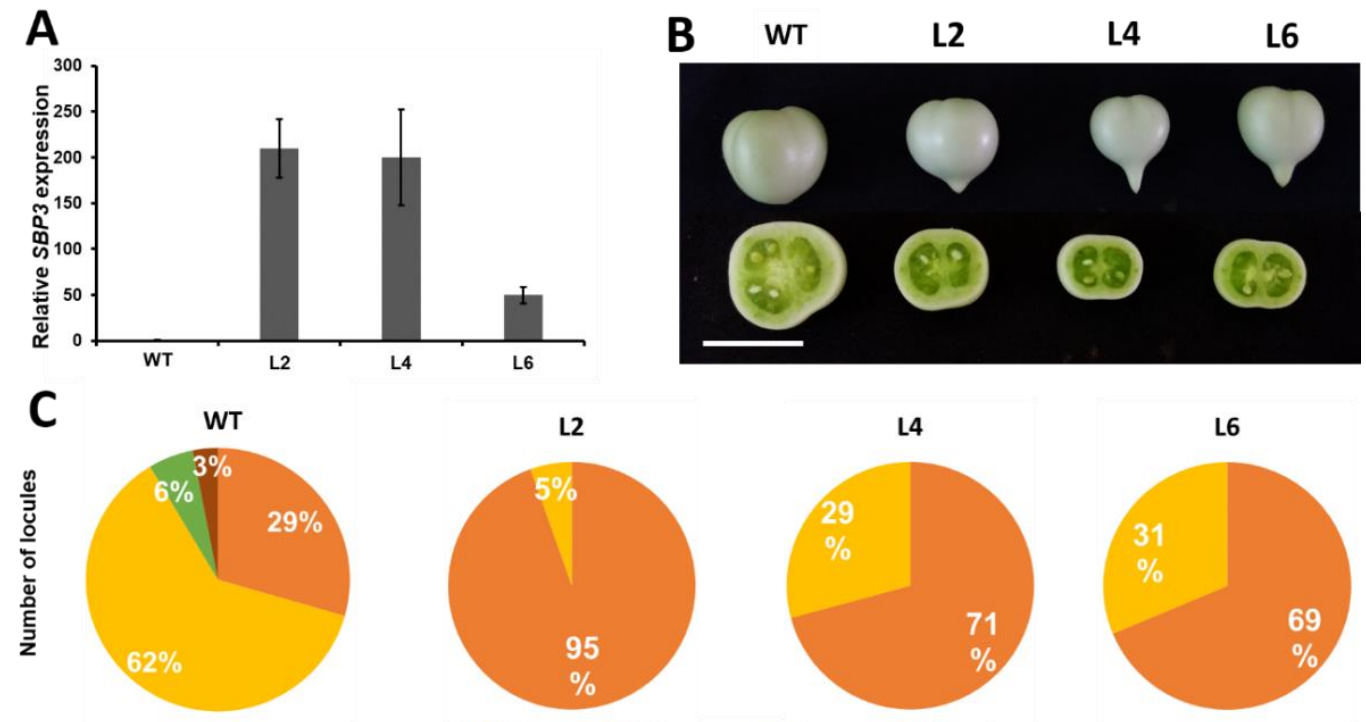

L2

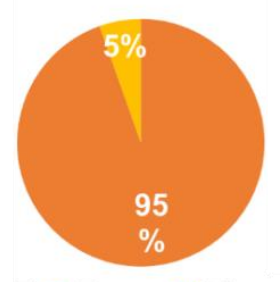

L4

L6

Two Three $\square$ Four $\square$ Five



Figure S2. Molecular and morphological characterization of rSBP3 overexpression lines. (A) Relative SBP3 transcript levels in leaves of each line. Tomato TUBULIN (Solyc04g081490) was used as an internal control for all experiments. qRT-PCR values are mean \pm s.e. of three biological replicates. (B) Representative fruits of WT, and rSBP3 lines L\#2, L\#4 and L\#6. Bar= 2cm (C) Percentage of fruits producing different number of locules in each genotype ( $\mathrm{n}=20$ plants). (D) Representative plants of each line. Bar $=7 \mathrm{~cm}$ 

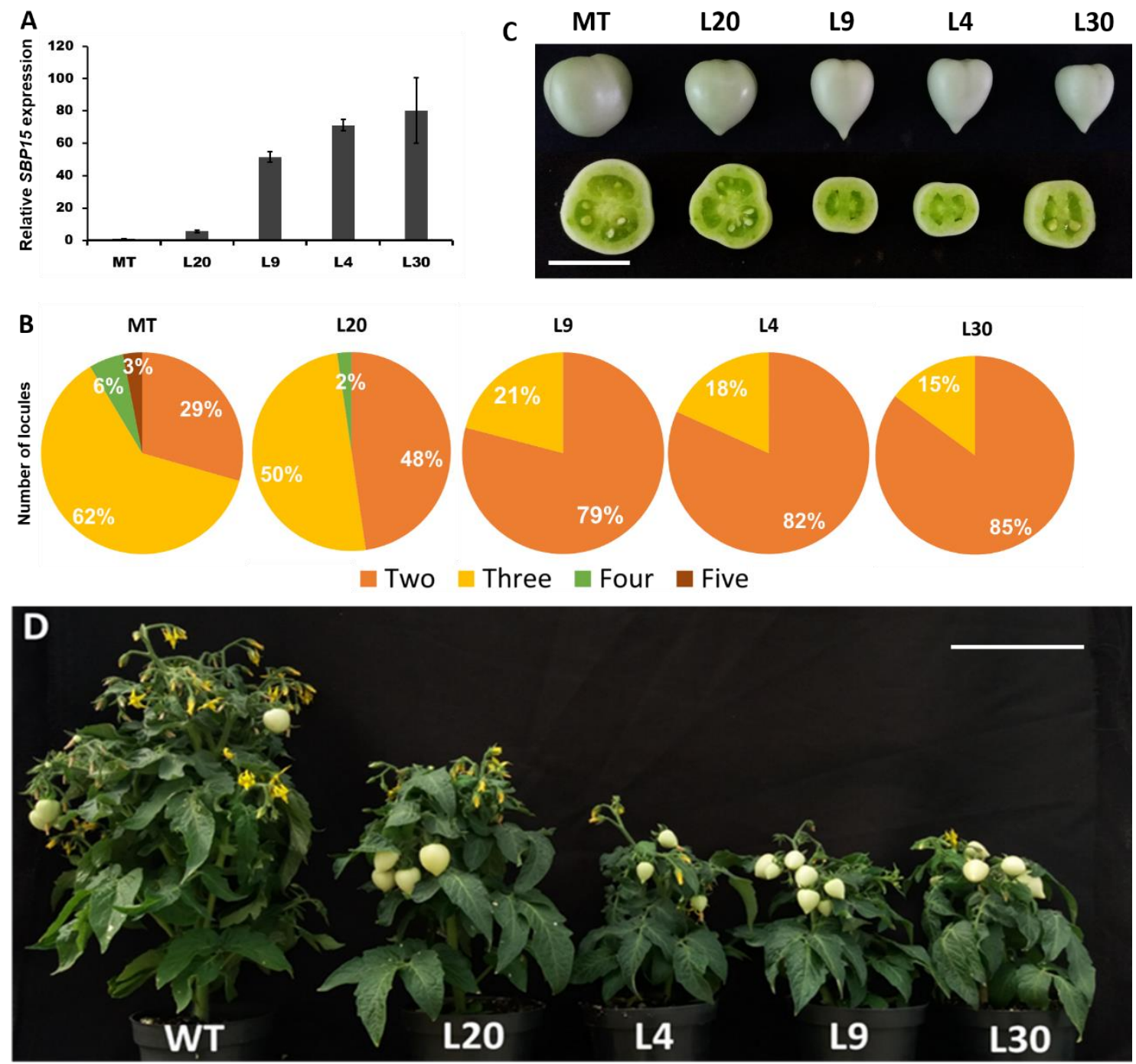

Two Three $\square$ Four $\square$ Five

Figure S3. Molecular and morphological characterization of rSBP15 overexpression lines. (A) Relative SBP15 transcript levels in leaves of each line. Tomato TUBULIN (Solyc04g081490) was used as an internal control for all experiments. qRT-PCR values are mean \pm s.e. of three biological replicates. (B) Representative fruits of WT, and rSBP15 lines L\#20, L\#4, L\#9 and L\#30. Bar= 2cm(C) Percentage of fruits producing different number of locules in each genotype. ( $\mathrm{n}=150$ fruits) (D) Representative plants of each line. $\mathrm{Bar}=7 \mathrm{~cm}$. 




Figure S4. Overexpression of miR156 in a background with mutated PRO or increased GA levels significantly alters plant architecture and fruit morphology. (A) Representative WT, pro, 156OE, and 156OE;pro plants. (B) Representative WT, 156OE, GA20oxOE and 156OE;GA20oxOE. Bars $=7 \mathrm{~cm}$. (C) Longitudinal section of $156 \mathrm{OE}$;pro and 156OE; GA20oxOE fruits showing no obvious locule structure. Bar $=1.5 \mathrm{~cm}$. 


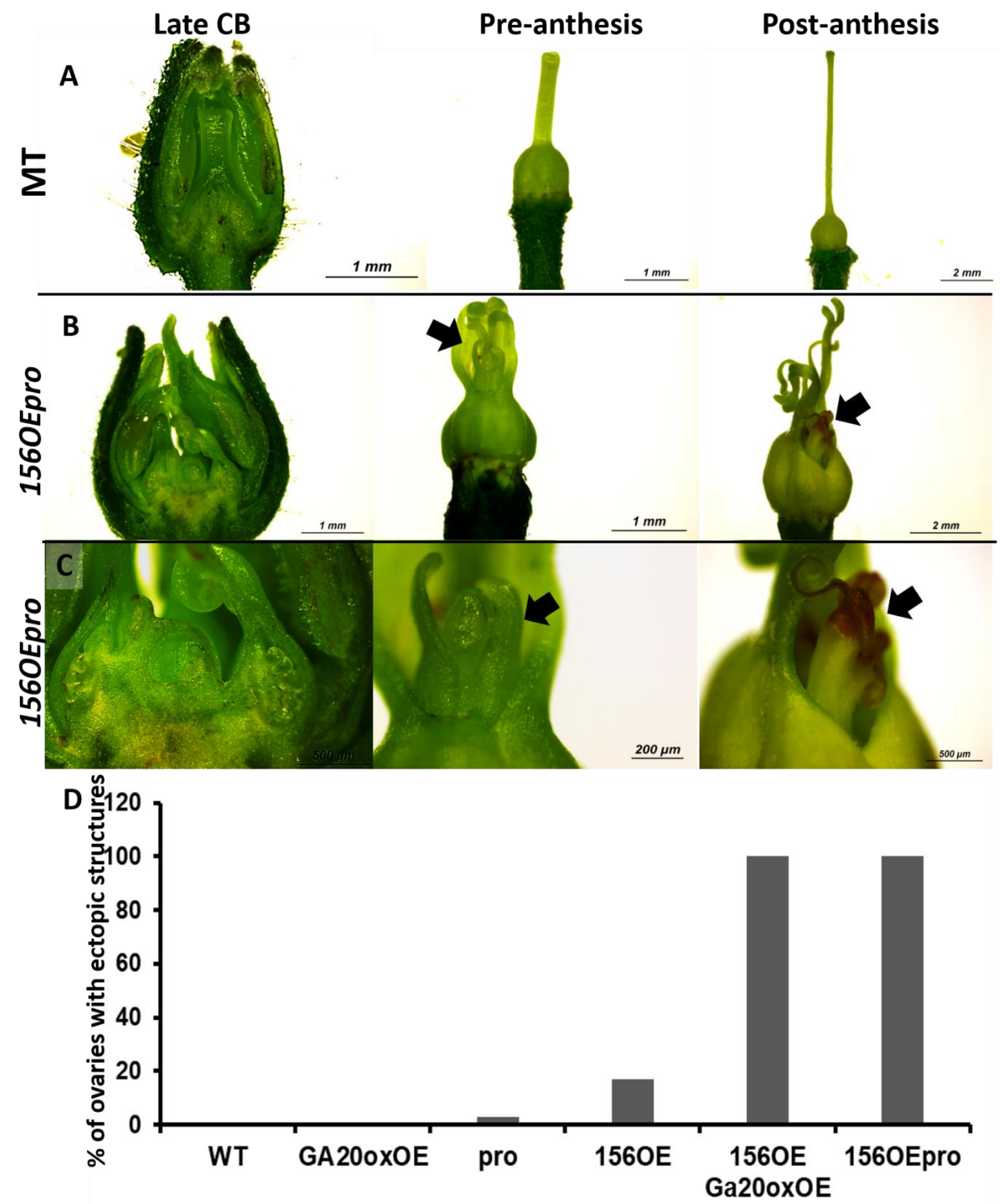

Figure S5. Stereomicroscope images of closed buds at later stages (in transverse sections), pre-anthesis ovaries and post-anthesis ovaries of MT (A) and 156OE;pro (B and C). (D) Percentage of the ovaries from the first inflorescence that showed indeterminate ectopic in each genotype. Black arrows indicate indeterminate ectopic structures growing from the ovary. 


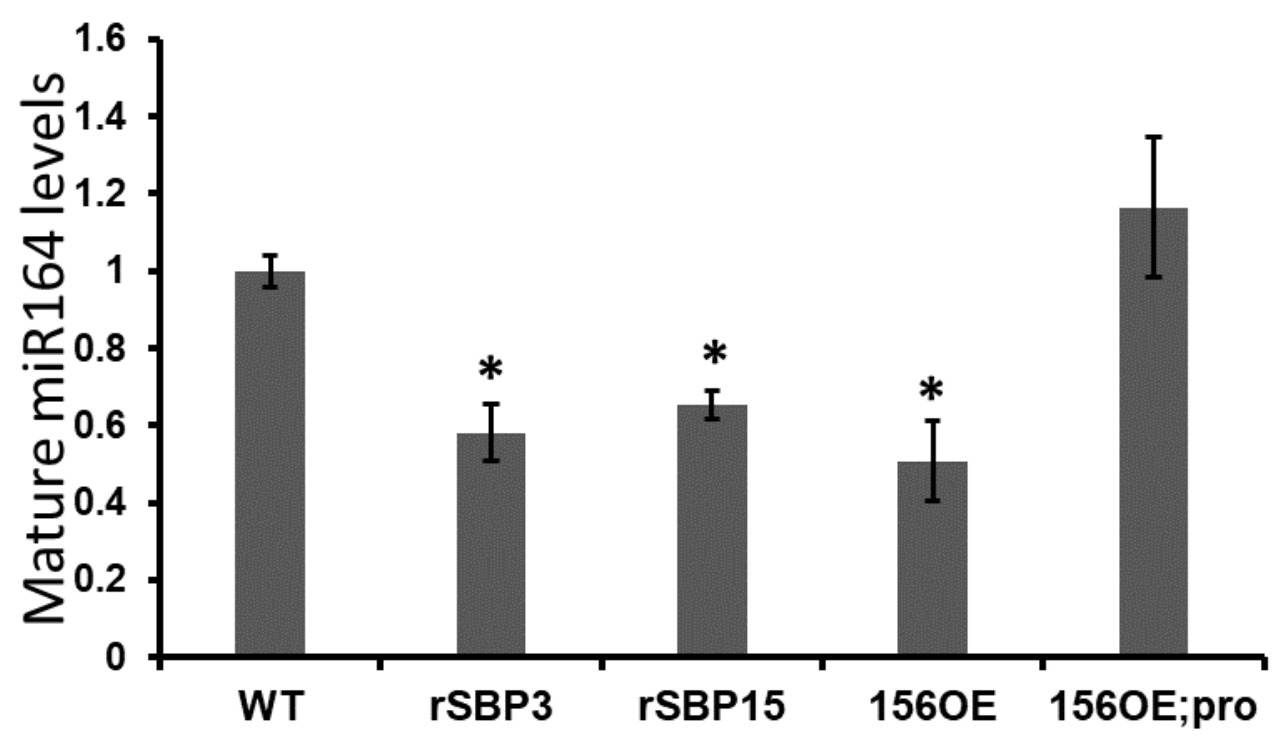

Figure S6 Mature miR164 levels in closed flower buds (CB) of W'T, rSBP3, rSBP15, 156OE and 156OE;pro. Tomato TUBULIN (Solyc04g-081490) was used as an internal control for all experiments. qRT-PCR values are mean \pm s.e. of three biological replicates. Asterisks indicate a significant difference when compared with reference sample according to Student's t test (two tailed; ${ }^{*} \mathrm{p}<0.05$ ). 



Figure S7. CLAUSA may be involved in $156 \mathrm{OE}$ phenotype, but not via SBP3 or SBP15. (A) clau mutant fruit phenotype. Bar $=2 \mathrm{~cm}(\mathrm{~B})$ and $(\mathrm{C})$ Relative CLAU expression in closed flower buds (CBs) of WT, 156OE, pro, 156OE;pro and two independent lines of and rSBP15. Tomato TUBULIN (Solyc04g081490) was used as an internal control for all experiments. qRT-PCR values are mean \pm s.e. of three biological replicates. Asterisks indicate a significant difference when compared with reference sample according to Student's t test (two tailed; ${ }^{*} \mathrm{p}<0.05$ ). 


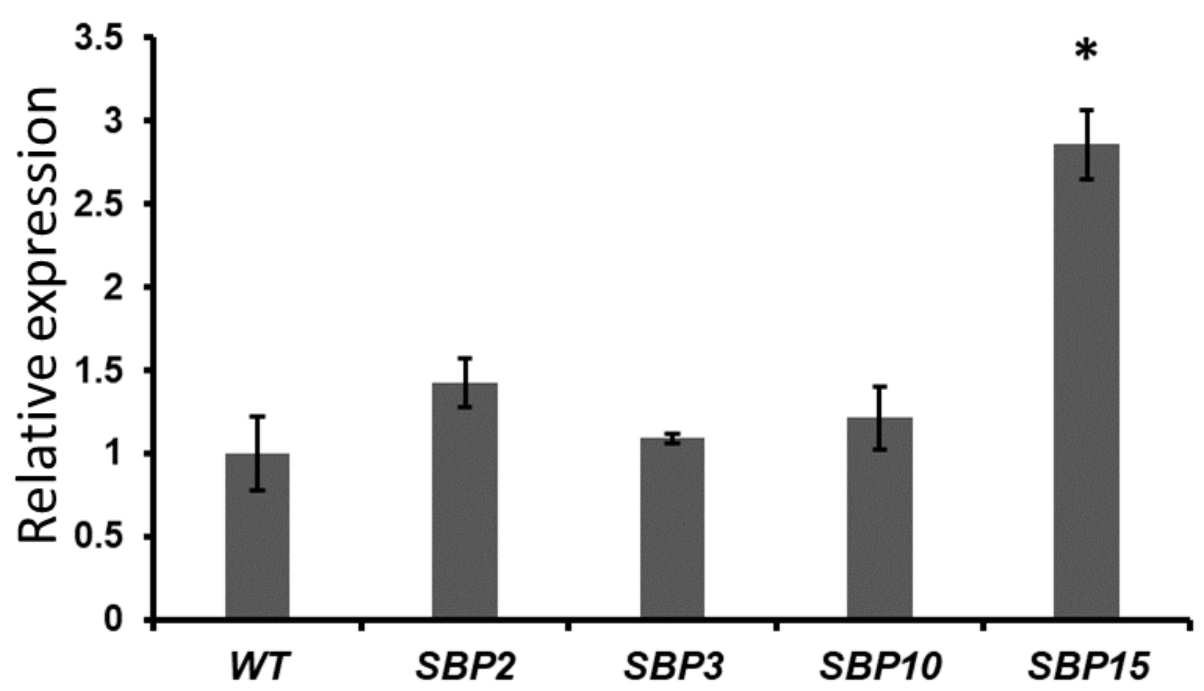

Figure S8. Expression of some miR156-targeted SBPs in procera closed flower buds. Tomato TUBULIN (Solyc04g081490) was used as an internal control for all experiments. qRT-PCR values are mean \pm s.e. of three biological replicates. Asterisks indicate a significant difference when compared with reference sample according to Student's t test (two tailed; ${ }^{*} \mathrm{p}<0.05$ ).

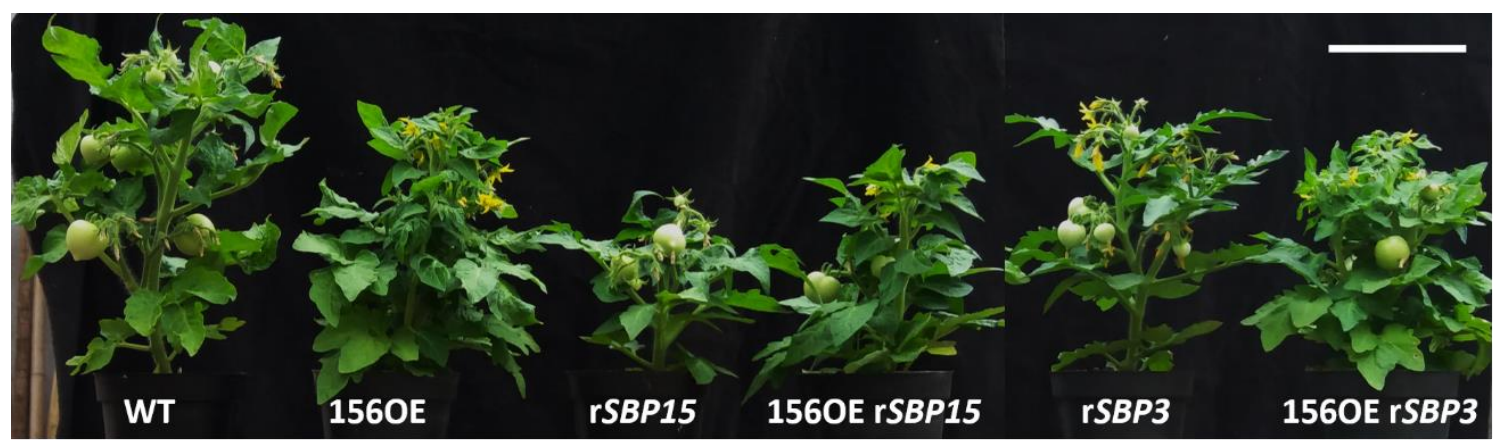

Figure S9. Representative WT, 156OE, rSBP15, 156OE;rSBP15, rSBP3 and 156OE;rSBP3 plants used in fruit and ovary morphological analysis. Bar $=7 \mathrm{~cm}$. 


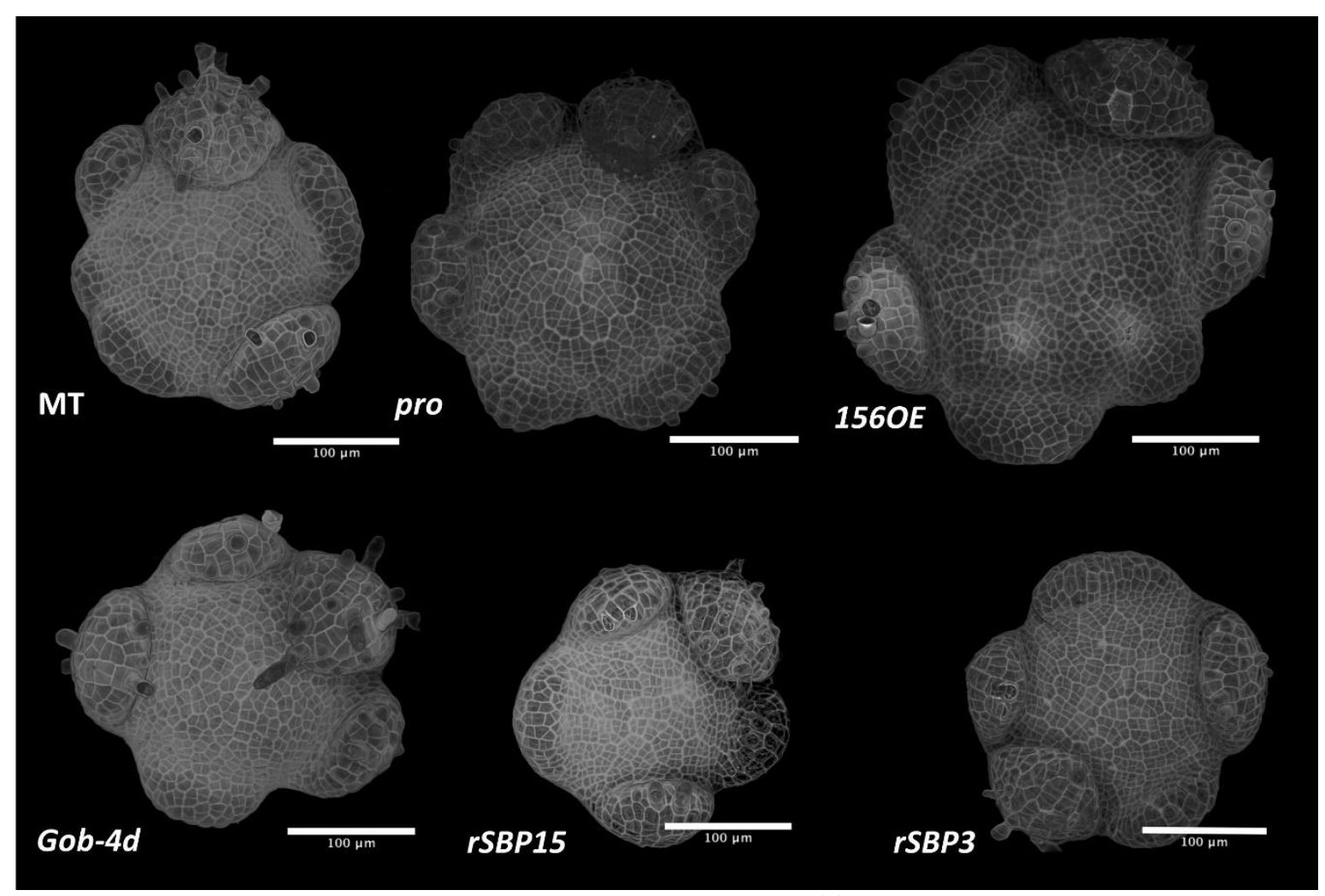

Figure S10. Raw 3D reconstruction of apexes stained by mPS-PI. The same representative meristems shown in Figure 7. 
Table 1. Oligonucleotide sequences used in this work

\begin{tabular}{|c|c|c|c|}
\hline Primer & Sequence & Locus ID & Utilization \\
\hline rSBP3F & CACCATGGACTCCGACAGCGTTTCG & Solyc10g009080 & Cloning \\
\hline rSBP3 R & TTACATGTGAAACACAGGATAG & Solyc10g009080 & Cloning \\
\hline $\begin{array}{l}\text { RSBP15 F1 } \\
\text { F }\end{array}$ & CACCATGGAACTGGGTTCAGTGTCT & Solyc10g078700 & Cloning \\
\hline rSBP15 F1 R & $\begin{array}{l}\text { GCTTAACAAGCTTAGAGCACCACTT } \\
\text { GAATCTGAGACTCCAG }\end{array}$ & Solyc10g078700 & Cloning \\
\hline rSBP15 F2 F & $\begin{array}{l}\text { GTGCTCTAAGCTTGTTAAGCAAT } \\
\text { CGGTCCTGGGGATCAAGG }\end{array}$ & Solyc10g078700 & Cloning \\
\hline rSBP15 F2 R & TCAAAGAGTCCAGTGCACATTCTG & Solyc10g078700 & Cloning \\
\hline GOB clon $\mathrm{F}$ & CACCGAGATTTATCATCAGATGCAGTTTG & Solyc07g062840 & Cloning \\
\hline GOB clon $\mathrm{R}$ & TCAGTAGCTCCACATACAGTCAAGT & Solyc07g062840 & Cloning \\
\hline PRO clon F & CACCAAGAGAGATCGAGATCGAGATCG & Solyc11g011260 & Cloning \\
\hline PRO clon & T'TACAACTCGACT'TCTCCGGC & Solyc11g011260 & Cloning \\
\hline GOB F & GGTTCTGGACTGCAACTTCAC & Solyc07g062840 & RT-qPCR \\
\hline GOB R & CCATTTTCGCTTTCCCAGG & Solyc07g062840 & RT-qPCR \\
\hline SBP3 F & CAAGTTGAACGGGCACCTAC & Solyc10g009080 & RT-qPCR \\
\hline SBP3 R & TGGCAAATGACAGAAGAGAGAG & Solyc10g009080 & RT-qPCR \\
\hline SBP15 F & GGTTCAGCTACCAGGACCAG & Solyc10g078700 & RT-qPCR \\
\hline SBP $15 \mathrm{R}$ & TGTGAACTTGGCTGTTGACC & Solyc10g078700 & RT-qPCR \\
\hline SBP2 F & ССССТTGTCСАСТСТАAАССТАС & Solyc04g045560 & RT-qPCR \\
\hline SBP2 R & GTCTGCATCTCAGTGGTCCCT & Solyc04g045560 & RT-qPCR \\
\hline SBP10 F & TTTTAATCGAGGTGCCAAGG & Solyc05g015510 & RT-qPCR \\
\hline SBP10 R & AACACTCTCAGGCTCGGATG & Solyc05g015510 & RT-qPCR \\
\hline miR164 F & ACTGATGTGGAGAAGCAGGGCA & MIMAT0000185 & RT-qPCR \\
\hline miR156 F & CCTGAGTGACAGAAGAGAGTG & MIMAT0000167 & RT-qPCR \\
\hline $\begin{array}{l}\text { Reverse } \\
\text { Universal }\end{array}$ & GTGCAGGGTCCGAGG & ---------------------- & RT-qPCR \\
\hline SITUB F & AACCTCCATTCAGGAGATGTTTT & Solyc04g081490 & RT-qPCR \\
\hline SITUB R & TCTGCTGTAGCATCCTGGTATT & Solyc04g081490 & RT-qPCR \\
\hline miR164 RT & $\begin{array}{l}\text { GTCGTATCCAGTGCAGGGTCCGAG } \\
\text { GTATTCGCACTGGATACGACTGCACG }\end{array}$ & MIMAT0000185 & $\begin{array}{l}\text { Reverse } \\
\text { Transcription }\end{array}$ \\
\hline miR156 RT & $\begin{array}{l}\text { GTCGTATCCAGTGCAGGGTCCGA } \\
\text { GGTATTCGCACTGGATACGACTGCTCT }\end{array}$ & MIMAT0000167 & $\begin{array}{l}\text { Reverse } \\
\text { Transcription }\end{array}$ \\
\hline
\end{tabular}




\section{REFERENCES}

Aida, M., Ishida, T., \& Tasaka, M. (1999). Shoot apical meristem and cotyledon formation during Arabidopsis embryogenesis: interaction among the CUP-SHAPED COTYLEDON and SHOOT MERISTEMLESS genes. Development (Cambridge, England), 126(8), 1563-1570.

Avivi, Y., Lev-Yadun, S., Morozova, N., Libs, L., Williams, L., Zhao, J., ... Grafi, G. (2000). Clausa, a tomato mutant with a wide range of phenotypic perturbations, displays a cell typedependent expression of the homeobox gene LeT6/TKn2. Plant Physiology, 124(2), 541-552. https://doi.org/10.1104/pp.124.2.541

Bar, M., Ben-Herzel, O., Kohay, H., Shtein, I., \& Ori, N. (2015). CLAUSA restricts tomato leaf morphogenesis and GOBLET expression. Plant Journal, 83(5), 888-902. https://doi.org/10.1111/tpj.12936

Bar, M., Israeli, A., Levy, M., Ben Gera, H., Jiménez-Gómez, J., Kouril, S., ... Ori, N. (2016). CLAUSA is a MYB Transcription Factor that Promotes Leaf Differentiation by Attenuating Cytokinin Signaling. The Plant Cell, 28(July), tpc.00211.2016. https://doi.org/10.1105/tpc.16.00211

Bassel, G. W., Mullen, R. T., \& Bewley, J. D. (2008). procera is a putative DELLA mutant in tomato (Solanum lycopersicum ): effects on the seed and vegetative plant, 59(3), 585-593. https://doi.org/10.1093/jxb/erm354

Bencivenga, S., Serrano-Mislata, A., Bush, M., Fox, S., \& Sablowski, R. (2016). Control of Oriented Tissue Growth through Repression of Organ Boundary Genes Promotes Stem $\begin{array}{llll}\text { Morphogenesis. } & \text { Developmental } & \text { 39(2), } & \text { 198-208. }\end{array}$ https://doi.org/10.1016/j.devcel.2016.08.013

Berger, Y., Harpaz-Saad, S., Brand, A., Melnik, H., Sirding, N., Alvarez, J. P., ... Ori, N. (2009). The NAC-domain transcription factor GOBLET specifies leaflet boundaries in compound tomato leaves. Development (Cambridge, England), 136(5), 823-832. https://doi.org/10.1242/dev.031625

Birkenbihl, R. P., Jach, G., Saedler, H., \& Huijser, P. (2005). Functional Dissection of the Plantspecific SBP-Domain: Overlap of the DNA-binding and Nuclear Localization Domains, 585-596. https://doi.org/10.1016/j.jmb.2005.07.013

Blein, T., Pulido, A., Vialette-Guiraud, A., Nikovics, K., Morin, H., Hay, A., ... Laufs, P. (2008). A conserved molecular framework for compound leaf development. Science (New York, N.Y.), 322(5909), 1835-1839. https://doi.org/10.1126/science.1166168 
Brukhin, V., Hernould, M., Gonzalez, N., Chevalier, C., \& Mouras, a. (2003). Flower development schedule in tomato Lycopersicon esculentum cv Sweet Cherry. Sex. Plant Reprod., 15(6), 311-320. https://doi.org/10.1007/s00497-003-0167-7

Cardon, G., Höhmann, S., Klein, J., Nettesheim, K., Saedler, H., \& Huijser, P. (1999). Molecular characterisation of the Arabidopsis SBP-box genes. Gene, 237(1), 91-104. https://doi.org/10.1016/S0378-1119(99)00308-X

Carrera, E., Ruiz-Rivero, O., Peres, L. E. P., Atares, a., \& Garcia-Martinez, J. L. (2012). Characterization of the procera Tomato Mutant Shows Novel Functions of the SIDELLA Protein in the Control of Flower Morphology, Cell Division and Expansion, and the AuxinSignaling Pathway during Fruit-Set and Development. Plant Physiology, 160(3), 1581-1596. https://doi.org/10.1104/pp.112.204552

Cheng, X., Peng, J., Ma, J., Tang, Y., Chen, R., Mysore, K. S., \& Wen, J. (2012). NO APICAL MERISTEM (MtNAM) regulates floral organ identity and lateral organ separation in Medicago truncatula. New Phytologist, 195(1), 71-84. https://doi.org/10.1111/j.14698137.2012.04147.x

Cheng, Y., Dai, X., \& Zhao, Y. (2007). Auxin Synthesized by the YUCCA Flavin Monooxygenases Is Essential for Embryogenesis and Leaf Formation in Arabidopsis. The Plant Cell Online, 19(8), 2430-2439. https://doi.org/10.1105/tpc.107.053009

Daviere, J.-M., \& Achard, P. (2013). Gibberellin signaling in plants. Development, 140(6), 11471151. https://doi.org/10.1242/dev.087650

Davière, J. M., Wild, M., Regnault, T., Baumberger, N., Eisler, H., Genschik, P., \& Achard, P. (2014). Class i TCP-DELLA interactions in inflorescence shoot apex determine plant height. Current Biology, 24(16), 1923-1928. https://doi.org/10.1016/j.cub.2014.07.012

De Lucas, M., Davière, J. M., Rodríguez-Falcón, M., Pontin, M., Iglesias-Pedraz, J. M., Lorrain, S., ... Prat, S. (2008). A molecular framework for light and gibberellin control of cell elongation. Nature, 451(7177), 480-484. https://doi.org/10.1038/nature06520

Feng, S., Martinez, C., Gusmaroli, G., Wang, Y., Zhou, J., Wang, F., ... Deng, X. W. (2008). Coordinated regulation of Arabidopsis thaliana development by light and gibberellins. Nature, 451(7177), 475-479. https://doi.org/10.1038/nature06448

Fouracre, J. P., \& Poethig, R. S. (2019). Role for the shoot apical meristem in the specification of juvenile leaf identity in Arabidopsis, 1-10. https://doi.org/10.1073/pnas.1817853116

Fuentes, S., Ljung, K., Sorefan, K., Alvey, E., Harberd, N. P., \& Østergaard, L. (2012). Fruit Growth in Arabidopsis Occurs via DELLA-Dependent and DELLA-Independent Gibberellin Responses. The Plant Cell, 24(10), 3982-3996. https://doi.org/10.1105/tpc.112.103192 
Garcia-Hurtado, N., Carrera, E., Ruiz-rivero, O., López-gresa, M. P., Hedden, P., Gong, F., \& Garcia-Martinez, J. L. (2012). The characterization of transgenic tomato overexpressing In Posidonia oceanica cadmium induces changes in DNA reveals induction of parthenocarpic methylation and chromatin patterning fruit growth, higher yield, and alteration of the gibberellin biosynth, 63(16), 5803-5813. https://doi.org/10.1093/jxb/err313

Giovannoni, J. J. (2004). Genetic regulation of fruit development and ripening. The Plant Cell, 16 Suppl, S170-S180. https://doi.org/10.1105/tpc.019158

Gomez, M. D., Barro-Trastoy, D., Escoms, E., Saura-Sánchez, M., Sánchez, I., Briones-Moreno, A., ... Perez-Amador, M. A. (2018). Gibberellins negatively modulate ovule number in plants. Development, 145(13), dev163865. https://doi.org/10.1242/dev.163865

Hasson, A., Plessis, A., Blein, T., Adroher, B., Grigg, S., Tsiantis, M., ... Laufs, P. (2011a). Evolution and diverse roles of the CUP-SHAPED COTYLEDON genes in Arabidopsis leaf development. The Plant Cell, 23(1), 54-68. https://doi.org/10.1105/tpc.110.081448

Hasson, A., Plessis, A., Blein, T., Adroher, B., Grigg, S., Tsiantis, M., .. Laufs, P. (2011b). Evolution and Diverse Roles of the CUP-SHAPED COTYLEDON Genes in Arabidopsis Leaf Development. The Plant Cell, 23(1), 54-68. https://doi.org/10.1105/tpc.110.081448

Hauvermale, A. L., Ariizumi, T., \& Steber, C. M. (2012). Gibberellin Signaling: A Theme and Variations on, 160(0850981), 83-92. https://doi.org/10.1104/pp.112.200956

Hendelman, A., Stav, R., Zemach, H., \& Arazi, T. (2013). The tomato NAC transcription factor SINAM2 is involved in flower-boundary morphogenesis. Journal of Experimental Botany, 64(18), 5497-5507. https://doi.org/10.1093/jxb/ert324

Hyun, Y., Vincent, C., Martinez-gallegos, R., Porri, A., Coupland, G., Vincent, C., ... Coupland, G. (2016). Multi-layered Regulation of SPL15 and Cooperation with SOC1 Integrate Endogenous Flowering Pathways at the Arabidopsis Shoot Meristem Article Multi-layered Regulation of SPL15 and Cooperation with SOC1 Integrate Endogenous Flowering Pathways at the Arabid, 254-266. https://doi.org/10.1016/j.devcel.2016.04.001

Ishida, T., Aida, M., Takada, S., \& Tasaka, M. (2000). Involvement of CUP-SHAPED COTYLEDON genes in gynoecium and ovule development in Arabidopsis thaliana. Plant \& Cell Physiology, 41(1), 60-67. https://doi.org/10.1093/pcp/41.1.60

James, P., Halladay, J., \& Craig, E. A. (1996). Genomic Libraries and a Host.

Jones, A. R., Forero-vargas, M., Withers, S. P., Smith, R. S., Traas, J., Dewitte, W., \& Murray, J. A. H. (2017). Cell-size dependent progression of the cell cycle creates homeostasis and flexibility of plant cell size. Nature Communications, 8, 1-13. https://doi.org/10.1038/ncomms15060 
Jung, J., Ju, Y., Seo, P. J., Lee, J., Park, C., \& Genomics, P. (2012). The SOC1-SPL module integrates photoperiod and gibberellic acid signals to control flowering time in Arabidopsis, 577-588. https://doi.org/10.1111/j.1365-313X.2011.04813.x

Laufs, P., Peaucelle, A., Morin, H., \& Traas, J. (2004). MicroRNA regulation of the CUC genes is required for boundary size control in Arabidopsis meristems. Development (Cambridge, England), 131(17), 4311-4322. https://doi.org/10.1242/dev.01320

Lee, B. H., Jeon, J. O., Lee, M. M., \& Kim, J. H. (2015). Genetic interaction between GROWTHREGULATING FACTOR and CUP-SHAPED COTYLEDON in organ separation. Plant Signaling and Behavior, 10(2). https://doi.org/10.4161/15592324.2014.988071

Lee, E. H., Byun, J. A. E. K., \& Wilding, J. (1985). A NEW GIBBERELLIN BIOSYNTHESIS INHIBITOR, PACLOBUTRAZOL (PPa33), CONFERS INCREASED SO/ TOLERANCE ON SNAP BEAN PLANTS, 25(3), 265-275.

Liu, M., Shi, Z., Zhang, X., Wang, M., Zhang, L., Zheng, K., .. Yang, D. (2019). Inducible overexpression of Ideal Plant Architecture1 improves both yield and disease resistance in rice. Nature Plants, 5, 389-400. https://doi.org/10.1038/s41477-019-0383-2

Livak, K. J., \& Schmittgen, T. D. (2001). Analysis of Relative Gene Expression Data Using RealTime Quantitative PCR and the $2 x \square \square$ C T Method, 408, 402-408. https://doi.org/10.1006/meth.2001.1262

Lombardi-crestana, S., Da Silva Azevedo, M., E, G. F. F., Pino, L. E., Appezzato-da-Gloria, B., Figueira, A., ... Peres, L. E. P. (2012). The tomato ( Solanum natural In Posidonia oceanica lycopersicum cadmium induces changes in DNA genetic variation and the DELLA mutant procera control the methylation patterning competence necessary to form adventitious roots and shoots, 63(15), 5689-5703. https://doi.org/10.1093/jxb/err313

Morea, E. G. O., Marques, E., Felipe, G., Valente, G. T., Hernan, C., Rojas, B., .. Nogueira, S. (2016). Functional and evolutionary analyses of the miR156 and miR529 families in land plants. BMC Plant Biology, 1-13. https://doi.org/10.1186/s12870-016-0716-5

Munos, S., Ranc, N., Botton, E., Berard, a., Rolland, S., Duffe, P., .. Causse, M. (2011). Increase in Tomato Locule Number Is Controlled by Two Single-Nucleotide Polymorphisms Located Near WUSCHEL. Plant Physiology, 156(4), 2244-2254. https://doi.org/10.1104/pp.111.173997

Muños, S., Ranc, N., Botton, E., Bérard, A., Rolland, S., Duffé, P., .. Causse, M. (2011). Increase in Tomato Locule Number Is Controlled by Two Single-Nucleotide Polymorphisms Located Near WUSCHEL. Plant Physiology, 156(4), 2244-2254. https://doi.org/10.1104/pp.111.173997 
Peaucelle, A., Morin, H., Traas, J., \& Laufs, P. (2007). Plants expressing a miR164-resistant CUC2 gene reveal the importance of post-meristematic maintenance of phyllotaxy in Arabidopsis. Development, 134(6), 1045-1050. https://doi.org/10.1242/dev.02774

Pérez-Pérez, J. M., Candela, H., \& Micol, J. L. (2009). Understanding synergy in genetic interactions. Trends in Genetics, 25(8), 368-376. https://doi.org/10.1016/j.tig.2009.06.004

Rieu, I., Ruiz-Rivero, O., Fernandez-Garcia, N., Griffiths, J., Powers, S. J., Gong, F., ... Hedden, P. (2008). The gibberellin biosynthetic genes AtGA20ox1 and AtGA20ox2 act, partially redundantly, to promote growth and development throughout the Arabidopsis life cycle. Plant Journal, 53(3), 488-504. https://doi.org/10.1111/j.1365-313X.2007.03356.x

Rodriguez-Leal, D., Xu, C., Kwon, C. T., Soyars, C., Demesa-Arevalo, E., Man, J., ... Lippman, Z. B. (2019). Evolution of buffering in a genetic circuit controlling plant stem cell proliferation. Nature Genetics, 51(May). https://doi.org/10.1038/s41588-019-0389-8

Rodríguez, G. R., Muños, S., Anderson, C., Sim, S.-C., Michel, A., Causse, M., ... van der Knaap, E. (2011). Distribution of SUN, OVATE, LC, and FAS in the tomato germplasm and the relationship to fruit shape diversity. Plant Physiology, 156(1), 275-285. https://doi.org/10.1104/pp.110.167577

Rubio-Somoza, I., Zhou, C.-M., Confraria, A., Martinho, C., von Born, P., Baena-Gonzalez, E., ... Weigel, D. (2014). Temporal control of leaf complexity by miRNA-regulated licensing of protein complexes. Current Biology: CB, 24(22), 2714-2719. https://doi.org/10.1016/j.cub.2014.09.058

Schwarz, S., Grande, Æ. A. V, Bujdoso, Æ. N., Saedler, H., \& Huijser, Æ. P. (2008). The microRNA regulated SBP-box genes SPL9 and SPL15 control shoot maturation in Arabidopsis, 183-195. https://doi.org/10.1007/s11103-008-9310-z

Serrano-Mislata, A., Bencivenga, S., Bush, M., Schiessl, K., Boden, S., \& Sablowski, R. (2017). DELLA genes restrict inflorescence meristem function independently of plant height. Nature Plants, 3(9), 749-754. https://doi.org/10.1038/s41477-017-0003-y

Serrano-Mislata, A., Schiessl, K., \& Sablowski, R. (2015). Active Control of Cell Size Generates Spatial Detail during Plant Organogenesis. Current Biology, 25(22), 2991-2996. https://doi.org/10.1016/j.cub.2015.10.008

Seymour, G. B., Østergaard, L., Chapman, N. H., Knapp, S., \& Martin, C. (2013). Fruit Development and Ripening. Annual Review of Plant Biology, 64(1), 219-241. https://doi.org/10.1146/annurev-arplant-050312-120057 
Shuping Xing, Maria Salinas, Susanne Hohmann, Rita Berndtgen, P. H. (2010). miR156-Targeted and Nontargeted SBP-Box Transcription Factors Act in Concert to Secure Male Fertility in Arabidopsis. The Plant Cell, 22(December), 3935-3950. https://doi.org/10.1105/tpc.110.079343

Sieber, P., Wellmer, F., Gheyselinck, J., Riechmann, J. L., \& Meyerowitz, E. M. (2007). Redundancy and specialization among plant microRNAs: role of the MIR164 family in developmental robustness. Development, 134(6), 1051-1060. https://doi.org/10.1242/dev.02817

Silva, G. F. F. E., Silva, E. M., Da Silva Azevedo, M., Guivin, M. A. C., Ramiro, D. A., Figueiredo, C. R., ... Nogueira, F. T. S. (2014). MicroRNA156-targeted SPL/SBP box transcription factors regulate tomato ovary and fruit development. Plant Journal, 78(4), 604618. https://doi.org/10.1111/tpj.12493

Silva, G. F. F., Silva, E. M., Correa, J. P. O., Vicente, M. H., Jiang, N., Notini, M. M., ... Nogueira, F. T. S. (2019). Tomato floral induction and flower development are orchestrated by the interplay between gibberellin and two unrelated microRNA-controlled modules. New Phytologist, 221(3), 1328-1344. https://doi.org/10.1111/nph.15492

Takada, S., Hibara, K., Ishida, T., \& Tasaka, M. (2001). The CUP-SHAPED COTYLEDON1 gene of Arabidopsis regulates shoot apical meristem formation. Development (Cambridge, England), 128(7), 1127-1135. Retrieved from http://dev.biologists.org/content/128/7/1127.abstract

Tian, C., Zhang, X., He, J., Yu, H., Wang, Y., Shi, B., ... Jiao, Y. (2014). An organ boundaryenriched gene regulatory network uncovers regulatory hierarchies underlying axillary meristem initiation. Molecular Systems Biology, 10(10), 755-755. https://doi.org/10.15252/msb.20145470

Usami, T., Horiguchi, G., Yano, S., \& Tsukaya, H. (2009). The more and smaller cells mutants of Arabidopsis thaliana identify novel roles for SQUAMOSA PROMOTER BINDING PROTEIN-LIKE genes in the control of heteroblasty. Development, 136(6), 955-964. https://doi.org/10.1242/dev.028613

Varkonyi-Gasic, E., Wu, R., Wood, M., Walton, E. F., \& Hellens, R. P. (2007). Protocol: a highly sensitive RT-PCR method for detection and quantification of microRNAs. Plant Methods, 3 , 12. https://doi.org/10.1186/1746-4811-3-12 
Vialette-Guiraud, Aurélie C. M., Chauvet, A., Gutierrez-Mazariegos, J., Eschstruth, A., Ratet, P., \& Scutt, C. P. (2016). A Conserved Role for the NAM/miR164 Developmental Module Reveals a Common Mechanism Underlying Carpel Margin Fusion in Monocarpous and Syncarpous Eurosids. Frontiers in Plant Science, 6(January), 1-11. https://doi.org/10.3389/fpls.2015.01239

Vialette-Guiraud, Aurlie C.M., Adam, H., Finet, C., Jasinski, S., Jouannic, S., \& Scutt, C. P. (2011). Insights from ANA-grade angiosperms into the early evolution of CUP-SHAPED COTYLEDON genes. Annals of Botany, 107(9), 1511-1519. https://doi.org/10.1093/aob/mcr024

Wang, J., Schwab, R., Czech, B., Mica, E., \& Weigel, D. (2008). Dual Effects of miR156-Targeted SPL Genes and CYP78A5 / KLUH on Plastochron Length and Organ Size in Arabidopsis thaliana, 20(May), 1231-1243. https://doi.org/10.1105/tpc.108.058180

Wang, J. W., Czech, B., \& Weigel, D. (2009). miR156-Regulated SPL Transcription Factors Define an Endogenous Flowering Pathway in Arabidopsis thaliana. Cell, 138(4), 738-749. https://doi.org/10.1016/j.cell.2009.06.014

Wang, Q., Hasson, A., Rossmann, S., \& Theres, K. (2016). Divide et impera: Boundaries shape the plant body and initiate new meristems. New Phytologist, 209(2), 485-498. https://doi.org/10.1111/nph.13641

Wu, G., Park, M. Y., Conway, S. R., Wang, J., Weigel, D., \& Scott, R. (2010). The sequential action of miR156 and miR172 regulates developmental timing in Arabidopsis. Cell, 138(4), 750-759. https://doi.org/10.1016/j.cell.2009.06.031.The

Wu, G., \& Poethig, R. S. (2006). Temporal regulation of shoot development in Arabidopsis thaliana by miR156 and its target SPL3, 3547, 3539-3547. https://doi.org/10.1242/dev.02521

Xing, S., Salinas, M., Garcia-Molina, A., Höhmann, S., Berndtgen, R., \& Huijser, P. (2013). SPL8 and miR156-targeted SPL genes redundantly regulate Arabidopsis gynoecium differential patterning. Plant Journal, 75(4), 566-577. https://doi.org/10.1111/tpj.12221

Xu, C., Liberatore, K. L., MacAlister, C. a, Huang, Z., Chu, Y.-H., Jiang, K., ... Lippman, Z. B. (2015a). A cascade of arabinosyltransferases controls shoot meristem size in tomato. Nature Genetics, 47(7), 784-792. https://doi.org/10.1038/ng.3309

Xu, C., Liberatore, K. L., MacAlister, C. a, Huang, Z., Chu, Y.-H., Jiang, K., ... Lippman, Z. B. (2015b). A cascade of arabinosyltransferases controls shoot meristem size in tomato. Nature Genetics, (May). https://doi.org/10.1038/ng.3309 
Yamaguchi, N., Winter, C. M., Wu, M.-F., Kanno, Y., Yamaguchi, A., Seo, M., \& Wagner, D. (2014). Gibberellin Acts Positively Then Negatively to Control Onset of Flower Formation in Arabidopsis. Science (New York, N.Y.), 344(5), 638-642.

Yu, S., Galvao, V. C., Zhang, Y.-C., Horrer, D., Zhang, T.-Q., Hao, Y.-H., ... Wang, J.-W. (2012). Gibberellin Regulates the Arabidopsis Floral Transition through miR156-Targeted SQUAMOSA PROMOTER BINDING-LIKE Transcription Factors. The Plant Cell, 24(8), 3320-3332. https://doi.org/10.1105/tpc.112.101014

Zsögön, A., Čermák, T., Naves, E. R., Notini, M. M., Edel, K. H., Weinl, S., ... Peres, P. (2018). De novo domestication of wild tomato using genome editing. Nature Publishing Group, (October). https://doi.org/10.1038/nbt.4272 